\title{
Review of the biomonitoring of persistent, bioaccumulative, and toxic substances in aquatic ecosystems of Mexico: 2001-2016
}

\author{
Eduardo Ramírez-Ayala ${ }^{1}$, Miguel A. Arguello-Pérez ${ }^{1}$, Adrián Tintos-Gómez ${ }^{1,2}$ \\ Rebeca Y. Pérez-Rodríguez ${ }^{3}$, Juan A. Díaz-Gómez ${ }^{2}$, Imelda Borja-Gómez ${ }^{4}$ \\ César Antonio Sepúlveda-Quiroz ${ }^{5}$ Manuel Patiño-Barragán ${ }^{4}$ \\ Carlos Lezama-Cervantes ${ }^{4}$ \& Joel Salomé-Baylón ${ }^{2}$ \\ ${ }^{1}$ Doctorate Program in Sciences in Biosystematics, Ecology and Management of Natural and Agricultural \\ Resources (BEMARENA), Department of Studies for the Development of the Coastal Zone \\ University of Guadalajara, Cihuatlán, Jalisco, Mexico \\ ${ }^{2}$ Renewable Energy Research Center, Technical Secretariat of the Academic Area \\ Technological University of Manzanillo, Manzanillo, Colima, Mexico \\ ${ }^{3}$ Department of Chemistry, Division of Natural and Exact Sciences \\ University of Guanajuato Guanajuato, Mexico \\ ${ }^{4}$ Faculty of Marine Sciences, University of Colima, Manzanillo, Colima, Mexico \\ ${ }^{5}$ Laboratory of Tropical Aquaculture, Academic Division of Biological Sciences (DACBiol) \\ Juárez Autonomous University of Tabasco, Villahermosa, Tabasco, México \\ Corresponding author: Adrián Tintos-Gómez (atintos48@gmail.com)
}

\begin{abstract}
Mexico is responsible for the protection and management of a large number and variety of aquatic bodies of national and international importance. Environmental pollution by so-called persistent and bioaccumulative toxic substances (PBTS) poses significant risks for all of the world's aquatic ecosystems, especially in countries with emerging economies, where environmental regulations are often poorly implemented. In Mexico, the development of industrial projects and the deficient application of environmental regulations, together with the rapid increase in population and the inefficient disposal of urban waste, have generated a severe problem of water pollution in the country. National environmental protection programs have not included the monitoring of PBTS, even though researchers have been monitoring the main aquatic ecosystems of the country for nearly three decades, generating valuable information that could help improve the protection and exploitation of these ecosystems. The present work reviewed a large portion of the available literature ( 150 articles) on aquatic biomonitoring of the main PBTS ( $\mathrm{Hg}, \mathrm{Cd}, \mathrm{Pb}, \mathrm{POCs}$, and PCBs) in Mexico. This work aims to collect, synthesize, and facilitate the management and interpretation of the reported data to improve the country's aquatic ecosystems' protection and management.
\end{abstract}

Keywords: PBTS; pollution; heavy metals; pesticides; biomonitoring; management; Mexico

\section{INTRODUCTION}

The chemical pollution of aquatic ecosystems, especially that caused by persistent and bioaccumulative toxic substances (PBTS), is one of the most critical problems facing modern society, both because of the important contribution to the overall degradation of aquatic ecosystems and the loss of biodiversity, but also because of the immediate risk posed for public health and food safety (Reyes et al., 2016). Creates a growing and multidimensional problem because aquatic ecosystems are the final destination of most PBTS derived from industrial, agricultural, and domestic activities, and this problem is expected to get more complicated by population and urban growth, as well as the accelerated growth of industrial activity around the world, especially in countries with emerging economies. Due to these countries' need for economicindustrial development, their governments often make important concessions to industrial sectors, sometimes

Corresponding editor: Sergio Contreras 
at the expense of the integrity of their aquatic ecosystems and natural resources in general. Some of these countries also have great ecological wealth, belonging to the group of countries considered megadiverse (11 countries that, together, harbor about $70 \%$ of Earth's biodiversity), including China, India, Brazil, Mexico, and Indonesia. Mexico, a leading member of this group, has many aquatic ecosystems of both national and international importance, being the second country with the highest number of wetlands included in the RAMSAR agreement (RAMSAR, 2019). It is also the eleventh most populous country on the planet, and even though its industrial development has lagged behind that of other emerging economies, several of the country's industrial sectors have been developed intensively, as is the case of the mining industry, the oil industry, the textile industry, and the agricultural industry.

Due to the complicated balance between economic development and ecological protection, several international conventions and treaties, laws, regulations, national policies, and monitoring programs (including the National Monitoring Network of National Waters Quality restructured in 1995; CONAGUA, 2017) have been implemented to promote the protection and conservation of the country's aquatic ecosystems. However, the complex contamination scenarios involving much of the country's aquatic ecosystems (CONAGUA, 2017) require other competent authorities (McCulligh, 2014). It has become clear that pollutant monitoring programs of aquatic ecosystems are essential to ensure compliance with national and international commitments (e.g., Minamata Treaty or Stockholm Convention, ratified by Mexico in 2003 and 2015, respectively) regarding the protection and responsible exploitation of these natural assets.

In Mexico, there is enough information about the short-term effects of aquatic pollutants on wild organisms, but there is very little information on their effects on human health, particularly of those effects that are associated with impacted ecosystems (ÁlvarezMoya \& Reinoso-Silva, 2015; Gómez-Meda et al., 2017). However, researchers have spent nearly three decades monitoring the concentration of the most important PBTS in various environmental matrices (water, sediment, air, and biota) of the country's aquatic ecosystems, generating valuable information that can serve as the basis for decision-making in environmental and public health matters, and help improve the protection and responsible management of aquatic ecosystems. This work aims to review the available studies on the biomonitoring of PBTS (mainly heavy metals, organochlorine pesticides, and polyaromatic hydrocarbons) in the various aquatic ecosystems of
Mexico between 2001 and 2016, presenting both the different concentrations of the main PBTS $(\mathrm{Hg}, \mathrm{Cd}, \mathrm{Pb}$, POC) presented as well as the organisms most commonly used in their monitoring.

\section{Selection of bibliographic material}

The selection of scientific publications was made through a search in different academic databases and search engines (Elsevier-Scopus, SCIELO, CONRICyT, and Google Scholar) of monitoring studies of PBTS in aquatic ecosystems in Mexico. The search was delimited using several combinations of keywords such as: "Mexico", "aquatic ecosystems", "lagoon", "estuary", "bay", "lake", "river", "wetland", "swamp", "pollution", "pollutant", "COPs", "heavy metals", "PCBs", "biota", "aquatic organisms", "fish", "bivalves", "clams", "crustaceans", "aquatic birds", among others, both in Spanish and in English. The search was carried out for the period from 2001 to 2016. For the selection of publications, only the articles and chapters of scientific books that met the following requirements were considered: 1) studies conducted in aquatic ecosystems of the Mexican territory, 2) studies on the concentration of heavy metals $(\mathrm{Hg}, \mathrm{Cd}$, and $\mathrm{Pb})$, organochlorine pesticides, polychlorinated biphenyls, and polyaromatic hydrocarbons, and 3) studies conducted in biota tissue (muscle, liver, blood, eggs).

\section{Selected studies}

Based on these criteria, 150 publications were selected. The number of studies selected in the present study is consistent with Páez-Osuna et al. (2017), who found 382 publications on general pollution for the Gulf of California area in the last 45 years. For practicality, we divided the country's aquatic ecosystems into four regions (Fig. 1): Gulf of California, Gulf of Mexican Caribbean, Mexican Pacific, and inland ecosystems (rivers and lakes-reservoirs). The average concentration obtained by each study in the calendar year of sampling was considered the annual average concentration to simplify the visualization and interpretation of the concentrations of PBTS in the present work. In the cases in which the authors did not indicate a single annual average for the calendar year in which the sampling was carried out, as, in the case of seasonal sampling (rain and dry season, or spring, summer, autumn, and winter), the data corresponding to that calendar year were selected and averaged using the following expression:

$$
\text { Annual average }=\sum_{1}^{i} \frac{x_{1} n_{1}+x_{2} n_{2}+\ldots+x_{i} n_{i}}{n_{1+} n_{2+\ldots}+n_{i}}
$$

where $\mathrm{x}_{1}, \mathrm{x}_{2}, \ldots \mathrm{x}_{i}$ are average concentrations within the corresponding calendar year, and $\mathrm{n}_{1}, \mathrm{n}_{2}, \ldots, \mathrm{n}_{i}$ are the sample sizes of the respective average. In the case in 


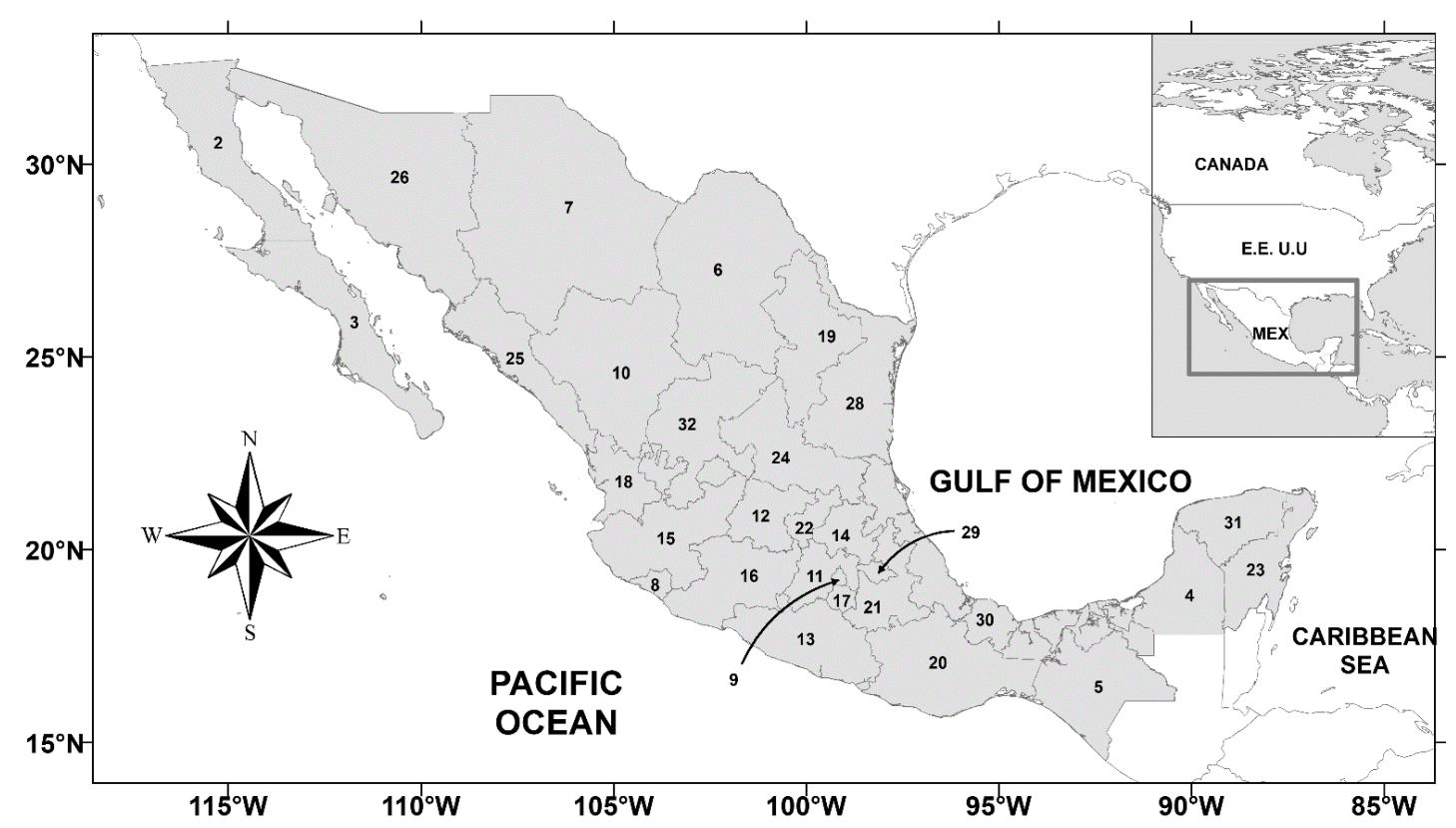

Figure 1. Mexican territory and political division. 1 Aguascalientes, 2 Baja California, 3 Baja California Sur, 4 Campeche, 5 Chiapas, 6 Chihuahua, 7 Coahuila, 8 Colima, 9 México Distrito Federal, 10 Durango, 11 Estado de México, 12 Guanajuato, 13 Guerrero, 14 Hidalgo, 15 Jalisco, 16 Michoacán, 17 Morelos, 18 Nayarit, 19 Nuevo León, 20 Oaxaca, 21 Puebla, 22 Querétaro, 23 Quintana Roo, 24 San Luis Potosí, 25 Sinaloa, 26 Sonora, 27 Tabasco, 28 Tamaulipas, 29 Tlaxcala, 30 Veracruz, 31 Yucatán, 32 Zacatecas.

which authors reported average concentrations for each sampling site within their study area, Equation 1 was used but taking $\mathrm{x}_{1}, \mathrm{x}_{2}, \ldots, \mathrm{x}_{i}$ as the averages of each sampling site and. $\mathrm{n}_{1}, \mathrm{n}_{2}, \ldots, \mathrm{n}_{i}$ as the sample sizes of the respective averages. Concerning persistent organic pesticides (POPs), which are found in the form $X=x_{1}$ $+\mathrm{x}_{2}, \ldots, \mathrm{x}_{\mathrm{n}}$ where $\mathrm{X}$ is the group of POPs and $\mathrm{x}$ are the metabolites, derivatives, or related compounds of $\mathrm{X}$, the total annual concentration was obtained using the following expression:

$$
\mathrm{X}=\sum_{1}^{i} \frac{x_{1} n_{1}+x_{2} n_{2}+\ldots+x_{i} n_{i}}{\frac{n_{1+} n_{2+\cdots}+n_{i}}{k}}
$$

where $\mathrm{x}_{1}, \mathrm{x}_{2}, \ldots, \mathrm{x}_{\mathrm{i}}$ are the averages of each of the derivatives, $\mathrm{n}_{1}, \mathrm{n}_{2}, \ldots, \mathrm{n}_{\mathrm{i}}$ are the sample sizes of the respective averages, and $\mathrm{k}$ is the number of derivatives of each group. When the authors presented more than one total annual concentration, Equation 1 was applied, substituting $\mathrm{x}$ for $\mathrm{X}$, and $\mathrm{n}$ for $\mathrm{k}$, applying the same temporal and spatial distribution criteria.

\section{Persistent pollutants in aquatic organisms}

Contamination of aquatic ecosystems with PBTS is one of the most critical environmental problems caused by direct human action (Saaristo et al., 2018) due to the great negative impact on wildlife. The adverse effects of pollution with PBTS can occur both in the short term (acute effects) and in the long term (chronic effects), including transgenerational effects. The effects of several of these pollutants (heavy metals and POPs mainly) at the sub-organism and organism level have been extensively studied for several decades and have been thoroughly described. PBTS are currently found in virtually all aquatic ecosystems in the world and, in most cases, below the probable effect concentrations established by environmental protection agencies, even below detec-tion limits. Thus, aquatic organisms are continuously exposed to a wide variety of concentrations and mixtures of these substances throughout their life cycle, and the real effects of this exposure cannot be appreciated in the short term or at basic levels of biological organization (Schwarzenbach et al., 2006). Nevertheless, the effects of chronic long-term exposure to low concentration levels are equally harmful; they are just less obvious (Saaristo et al., 2018). These effects may occur at the genetic, epigenetic, or behavioral level, affecting present or future gene-rations, compromising long-term survival and repro-duction, and decimating and damaging existing populations.

Since a large part of the world's population depends on the consumption of fishery products (marine and freshwater, animal and vegetable) as an essential part of their daily diet and due to this fact, the ability of PBTS to bioaccumulate and biomagnify in the trophic 
networks of aquatic ecosystems pose a serious public health problem, mainly in developing countries (FAO, 2018). The consumption of fishery products is considered the primary source of exposure to PBTS in humans (Dórea, 2008). Although the effects of poisoning with food contaminated with PBTSs have been well established, while the long-term effects of the consumption of fishery products contaminated with low concentrations of PBTS are still being studied. These effects may include neurological, cardiovascular, endocrine, or immunological alterations, but further studies are required (Dórea, 2008). The biomonitoring of PBTS in aquatic ecosystems is thus a vital tool for protecting wildlife and public health, providing valuable information on these substances' bioavailability and the spatial and temporal variation of these substances' bioavailable fraction pollutants. For an organism to have potential as a biomonitor, it must possess a series of desirable attributes (both ecological and logistical) to facilitate its application. A wide variety of organisms are currently used as biomonitors of aquatic pollution, including fish, bivalves, crustaceans, macroalgae, birds, or mammals.

\section{Heavy metals in biota}

The presence, bioaccumulation, and biomagnification of heavy metals in aquatic ecosystems have been widely studied in recent decades because the presence and bioaccumulation of some of these metals in trophic networks can pose a serious risk to human health (Mendoza-Carranza et al., 2016). The potential for bioaccumulation and biomagnification of heavy metals is high, and such a process can be complex and difficult to understand. It is not yet clear how some metals biomagnify the trophic networks of aquatic ecosystems, while the biomagnification of $\mathrm{Hg}$ and $\mathrm{Se}$ is an easy process to elucidate; other cases, such as $\mathrm{Cd}$ and $\mathrm{Pb}$, are still under discussion (Schneider et al., 2018). Bioaccumulation and bioconcentration processes are influenced by the exposure routes and by intraspecific and interspecific differences; thus, it is recommended that biomonitoring studies use several different species to make a better estimation of the behavior of pollutants in ecosystems (Jara-Marini et al., 2013). In general, monitoring the concentration of metals in aquatic organisms can make evident the pollution status of an ecosystem and help understand the potential risk to consumers (Wang et al., 2013).

Different organisms were used for monitoring the concentration of $\mathrm{Hg}, \mathrm{Cd}$, and $\mathrm{Pb}$ in different aquatic ecosystems in Mexico (Tables 1-2). Based on the information obtained in the present review, coastal ecosystems have been the most studied in this regard, mainly the coastal ecosystems of the Gulf of California.
The organisms used as biomonitors include various groups, such as macroalgae, bivalve mollusks, fish, sharks, rays, birds, and turtles. Bivalves and fish were the most frequently used, maybe because bivalves are frequently used as biomonitors throughout the world (Páez-Osuna \& Osuna-Martínez, 2011). They have various desirable qualities for aquatic biomonitoring, such as resistance to handling (which means they can be used for both in situ and ex situ studies), simple eating habits, they are efficient accumulators, sedentary, abundant, and cosmopolitan. However, even though they are found virtually on all Mexican coastal sites, their use as biomonitors has been limited to the Gulf of California area, mainly the States of Sonora and Sinaloa coasts. Furthermore, in some areas of the central Mexican Pacific (Jalisco-Colima-Michoacán), the northern part of the Gulf of Mexico (Tamaulipas), and the Mexican Caribbean, the number of studies using bivalves within the reviewed period are significantly scarce. Regarding the bivalve species used, the genus Crassostrea was the most common, probably due to its commercial importance, given that these species are extensively cultivated in coastal lagoons of the Gulf of California, which would have simplified many logistical issues during the sampling campaigns.

The use of teleost fish as biomonitors of aquatic pollution is also of great importance. These fish are found in virtually all aquatic ecosystems throughout the world, and many species can be considered integrators of ecological conditions, especially those closest to the highest levels of the food chain. Since they perform the function of transferring energy from the low to the high levels of the trophic networks of aquatic ecosystems (Van der Oost et al., 2003), they are a good mirror of the health of the ecosystem (Sedeño-Díaz \& LópezLópez, 2012). Fish are the organisms most frequently consumed by humans, especially those in high trophic levels and by bioaccumulating heavy metals (such as $\mathrm{Cd}, \mathrm{Pb} \mathrm{Hg}$ ), they passed them on to consumers and can cause both acute (poisoning) and chronic diseases, most of which are not yet fully understood. Unlike bivalves, fish as biomonitors of aquatic pollution is more widespread throughout the Mexican territory, including coastal and inland ecosystems. The present review shows that pollution monitoring studies have used different species of fish belonging to different trophic levels and with different eating habits, including planktivorous fish (sardines), detritivorous fish (lizas), omnivorous fish (catfish), carnivorous-piscivorous fish (mojarras, snooks, snappers), carnivorous-benthic fish and major pelagic fish. The most used species were the lizas (Mugilidae), probably due to their commercial importance in Mexico (Frías-Espiricueta et al., 2011). 
Table 1. Average concentration of $\mathrm{Hg}$ (in $\mu \mathrm{g} \mathrm{g}^{-1} \mathrm{dw}$ ) in different tissues of organisms from various aquatic ecosystems of Mexico. EU: Estero Urías; AEP: Altata-Ensenada del Pabellón; BGY: Bahía Guaymas; GCFR: Golfo de California; GSNR: Granjas-Sonora; GSNL: Granjas Sinaloa; GNYR: Granjas Nayarit; BBP: Bahía de Bacochibampo; BCT: Bahía de Cueta; BBCN: Bocana; GYEMP: Guaymas-Empalme LTM: Laguna de Términos; LTB: Laguna El Tobari; LCL: El Colorado; ERY: Estero El Rey; SMOT: Santa María-Ohurias-Topolobampo; SMR: Santa María- LA Reforma; LCHP: Lago de Chapala; BKK: Bahía de Kuu Kaak; BGMS: Bahía Las Guácimas; LAV: Laguna Alvarado; CSNR: Costa de Sonora; BTBP: Bahía de Topolobampo; BAP: Laguna Agiabampo; BCHC: Bachoco; CSC: Ciénega Santa Clara; LBN: Laguna Barra de Navidad; SBRB: Santa Bárbara; RPL: Río Palizada; RCHP: Río Chumpán; BTPB: Bahía de Topolobampo; BCMCH: Boca Camichín; BCHM: Bahía Chetumal; PLL: Presa Luis León; CSDR: Cañón del sumidero; MTCL: Mercado de Tecuala; RCL: Río Colorado; RCDLR: Río Candelaria; BCY: Bahía La Choya; ESBL: Estero de San Blas; LCTC: Lago de Catemaco; BLB: Bahía de Lobos; RMYR: El Mayor; RUSMT: Río Usumacinta; TCPN: Teacapán; PVM: Presa Viejo Mandín; CCP: Cucapa; MTTN: Minatitlán; RSTG: Río Santiago; TLCP: Tlacotalpan; URTCP: Urías-Teacapán; PVCZ: Puerto de Veracruz; CSPT: Cospita; CBCF: Baja California; PBLCH: Punta Belcher; IMGD: Isla Magdalena; PPG: El Portugués; BCHY: El Choyudo; PRY: Playa del Rey; DTLL: Dautillos; PCHL: Puerto Chale; RCHPT: Río Champotón; LPNCLT: Los Petenes-Celestún; RHND: Río Hondo; CGRR: Costa de Guerrero; CSNL: Costa de Sinaloa; PDBC: Península de Baja California; PEPA: Playa la Escobilla-Puerto Escondido-Puerto Ángel. SON: Sonora; SIN: Sinaloa; NAY: Nayarit; JAL: Jalisco; COL: Colima; MIC: Michoacán; GRO: Guerrero; OAX: Oaxaca; CHP: Chiapas; CAM: Campeche; ROO: Quintana Roo; YUC: Yucatán; TAB: Tabasco; VER: Veracruz; TAM: Tamaulipas; BCS: Baja California Sur; BCN: Baja California Norte; AGU: Aguascalientes; MEX: México; CMX: Ciudad de México. N/E: not specified; ND: not detect; -: not analized; D: dry weight; W: wet weight; M: muscle; ST: soft tissue; T: whole body; S: blood; B: biopsy; H: eggs.

\begin{tabular}{|c|c|c|c|}
\hline Species & Place & $\mathrm{Hg}$ & Reference \\
\hline \multicolumn{4}{|l|}{ Primary producers } \\
\hline Fitoplankton & UR (SIN) & $0.02^{\mathrm{D}}(2006)$ & Jara-Marini et al. (2012) \\
\hline Gracilaria sp. & UR (SIN) & $0.02^{\mathrm{D}}(2006)$ & Jara-Marini et al. (2012) \\
\hline Gracilaria subsecuentata & BGY (SON) & $0.09(1998)$ & Green-Ruiz et al. (2005) \\
\hline Chaetomorpha linum & UR (SIN) & $0.04^{\mathrm{D}}(2006)$ & Jara-Marini et al. (2012) \\
\hline Caulerpa serticularioides & UR (SIN) & $0.02^{\mathrm{D}}(2006)$ & Jara-Marini et al. (2012) \\
\hline Ulva Lactuca & BGY (SON) & $0.05^{\mathrm{D}}(1998)$ & Green-Ruiz et al. (2005) \\
\hline \multicolumn{4}{|l|}{ Mollusks } \\
\hline \multirow[t]{14}{*}{ Crassostrea corteziensis } & $\mathrm{BBP}(\mathrm{SON})$ & $0.04^{\mathrm{W}}(2004) \mathrm{ST}$ & García-Rico et al. (2010) \\
\hline & & $0.03^{\mathrm{W}}(2005) \mathrm{ST}$ & García-Rico et al. (2010) \\
\hline & UR (SIN) & $0.05^{\mathrm{D}}(2006) \mathrm{ST}$ & Jara-Marini et al. (2012) \\
\hline & & $0.17^{\mathrm{D}}(2008) \mathrm{ST}$ & Páez-Osuna \& Osuna-Martínez (2015) \\
\hline & $\mathrm{BCT}(\mathrm{SIN})$ & $0.37^{\mathrm{D}}(\mathrm{N} / \mathrm{E}) \mathrm{ST}$ & Osuna-Martínez et al. (2010) \\
\hline & & $0.57^{\mathrm{D}}(2008) \mathrm{ST}$ & Páez-Osuna \& Osuna-Martínez (2015) \\
\hline & LTB (SON) & $0.43^{\mathrm{D}}(2009) \mathrm{ST}$ & Jara-Marini et al. (2013) \\
\hline & LCL (SIN) & $0.54^{\mathrm{D}}(2008) \mathrm{ST}$ & Páez-Osuna \& Osuna-Martínez (2015) \\
\hline & SMOT (SIN) & $0.18^{\mathrm{D}}(2008) \mathrm{ST}$ & Páez-Osuna \& Osuna-Martínez (2015) \\
\hline & INM (SIN) & $0.39^{\mathrm{D}}(2008) \mathrm{ST}$ & Páez-Osuna \& Osuna-Martínez (2015) \\
\hline & SMR (SIN) & $0.30^{\mathrm{D}}(2008) \mathrm{ST}$ & Páez-Osuna \& Osuna-Martínez (2015) \\
\hline & $\mathrm{AEP}(\mathrm{SIN})$ & $0.52^{\mathrm{D}}(2008) \mathrm{ST}$ & Páez-Osuna \& Osuna-Martínez (2015) \\
\hline & ERY (SIN) & $0.29^{\mathrm{D}}(2008) \mathrm{ST}$ & Páez-Osuna \& Osuna-Martínez (2015) \\
\hline & UR (SIN) & $0.05^{\mathrm{D}}(2006) \mathrm{ST}$ & Jara-Marini et al. (2008) \\
\hline \multirow[t]{7}{*}{ Crassostrea gigas } & BGY (SON) & $0.23(1998) \mathrm{ST}$ & Green-Ruiz et al. (2005) \\
\hline & LTB (SON) & $0.39^{\mathrm{D}}(2009)$ & Jara-Marini et al. (2013) \\
\hline & LCL (SIN) & $0.93^{\mathrm{D}}(\mathrm{N} / \mathrm{E}) \mathrm{ST}$ & Osuna-Martínez et al. (2010) \\
\hline & BCT (SIN) & $0.46^{\mathrm{D}}(\mathrm{N} / \mathrm{E}) \mathrm{ST}$ & Osuna-Martínez et al. (2010) \\
\hline & SMR (SIN) & $0.24^{\mathrm{D}}(\mathrm{N} / \mathrm{E}) \mathrm{ST}$ & Osuna-Martínez et al. (2010) \\
\hline & AEP (SIN) & $0.23^{\mathrm{D}}(\mathrm{N} / \mathrm{E}) \mathrm{ST}$ & Osuna-Martínez et al. (2010) \\
\hline & CSNR (SON) & $0.03^{\mathrm{W}}(1997) \mathrm{ST}$ & Garcia-Rico \& Ramos-Ruiz (2001) \\
\hline \multirow[t]{4}{*}{ Crassostrea virginica } & LTM (CAM) & $0.0001(2011) \mathrm{ST}$ & Benítez et al. (2012) \\
\hline & RPL (CAM) & $0.5(\mathrm{~N} / \mathrm{E}) \mathrm{ST}$ & Aguilar et al. (2012) \\
\hline & RCHP (CAM) & $0.7(\mathrm{~N} / \mathrm{E}) \mathrm{ST}$ & Aguilar et al. (2012) \\
\hline & RCDLR (CAM) & $1.1(\mathrm{~N} / \mathrm{E}) \mathrm{ST}$ & Aguilar et al. (2012) \\
\hline \multirow[t]{4}{*}{ Crassostrea sp. } & $\mathrm{BCHC}(\mathrm{SON})$ & $0.17^{\mathrm{D}}(2010-2011) \mathrm{ST}$ & Delgado-Álvarez et al. (2015b) \\
\hline & SBRB (SON) & $0.25^{\mathrm{D}}(2010-2011) \mathrm{ST}$ & Delgado-Álvarez et al. (2015b) \\
\hline & LTB (SON) & $0.14^{\mathrm{D}}(2010-2011) \mathrm{ST}$ & Delgado-Álvarez et al. (2015b) \\
\hline & $\mathrm{BAP}(\mathrm{SON})$ & $0.17^{\mathrm{D}}(2010-2011) \mathrm{ST}$ & Delgado-Álvarez et al. (2015b) \\
\hline
\end{tabular}


continuation

\begin{tabular}{|c|c|c|c|}
\hline Species & Place & $\mathrm{Hg}$ & Reference \\
\hline & SMR (SIN) & $0.19^{\mathrm{D}}(2010-2011) \mathrm{ST}$ & Delgado-Álvarez et al. (2015b) \\
\hline & BCMCH (NAY) & $0.19^{\mathrm{D}}(2010-2011) \mathrm{ST}$ & Delgado-Álvarez et al. (2015b) \\
\hline & CGC (N/A) & $0.17^{\mathrm{D}}(2010-2011) \mathrm{ST}$ & Delgado-Álvarez et al. (2015b) \\
\hline Megapitaria squalida & BKK (SON) & 0.001 (2003) ST & García-Hernández et al. (2005) \\
\hline \multirow[t]{2}{*}{ Mytella strigata } & UR (SIN) & $0.06^{\mathrm{D}}(2006) \mathrm{ST}$ & Jara-Marini et al. (2012) \\
\hline & UR (SIN) & $0.06^{\mathrm{D}}(2006) \mathrm{ST}$ & Jara-Marini et al. (2008) \\
\hline Chione fluctifraga & LTB (SON) & $0.28^{\mathrm{D}}$ (2009) ST & Jara-Marini et al. (2013) \\
\hline Chione gnidia & LTB (SON) & $0.47^{\mathrm{D}}(2009) \mathrm{ST}$ & Jara-Marini et al. (2013) \\
\hline Chione californiensis & BKK (SON) & $0.001^{\mathrm{W}}(2003) \mathrm{ST}$ & García-Hernández et al. (2005) \\
\hline Chione subrugosa & BGY (SON) & $0.06(1998) \mathrm{ST}$ & Green-Ruiz et al. (2005) \\
\hline Chione sp. & DRC (SON) & $0.04^{\mathrm{D}}(1998-2000) \mathrm{ST}$ & García-Hernández et al. (2001) \\
\hline Anadara tuberculosa & LTB (SON) & $0.20^{\mathrm{D}}(2009) \mathrm{ST}$ & Jara-Marini et al. (2013) \\
\hline Corbicula sp. & RCL (SON) & $0.04^{\mathrm{D}}(1998-2000) \mathrm{ST}$ & García-Hernández et al. (2001) \\
\hline Corbicula fluminea & RCZ (VER) & $\left.0.09^{\mathrm{D}} 2005\right) \mathrm{ST}$ & Ruelas-Inzunza et al. (2009a) \\
\hline Polymesoda caroliniana & RCZ (VER) & $0.155^{\mathrm{D}}(2005) \mathrm{ST}$ & Ruelas-Inzunza et al. (2009a) \\
\hline Polymesoda caroliniana & RCZ (VER) & $0.115^{\mathrm{D}}(2006) \mathrm{ST}$ & Ruelas-Inzunza et al. (2009a) \\
\hline Pinna rugosa & $\mathrm{BKK}(\mathrm{SON})$ & $0.022^{\mathrm{W}}(2003) \mathrm{ST}$ & García-Hernández et al. (2005) \\
\hline Hexaplex erythrostomus & BKK (SON) & $0.05^{\mathrm{W}}(2003) \mathrm{ST}$ & García-Hernández et al. (2005) \\
\hline Mytilopsis sallei & $\mathrm{BCHM}(\mathrm{ROO})$ & 1.09 (2002) ST & Díaz-López et al. (2006) \\
\hline \multicolumn{4}{|l|}{ Crustaceans } \\
\hline \multirow[t]{6}{*}{ Penaeus vannamei } & AEP (SIN) & $0.20^{\mathrm{D}}(1998-1999) \mathrm{M}$ & Ruelas-Inzunza et al. (2004) \\
\hline & $\mathrm{UR}(\mathrm{SIN})$ & $0.03^{\mathrm{D}}(2006) \mathrm{T}$ & Jara-Marini et al. (2012) \\
\hline & AEP (SIN) & $0.06^{\mathrm{W}}(1998-1999) \mathrm{M}$ & Ruelas- Inzunza et al. (2011a) \\
\hline & GSNR (SON) & $0.19(2010) \mathrm{M}$ & Delgado-Álvarez et al. (2015a) \\
\hline & GSNL (SIN) & $0.31(2010) \mathrm{M}$ & Delgado-Álvarez et al. (2015a) \\
\hline & GNYR (NAY) & $0.45(2010) \mathrm{M}$ & Delgado-Álvarez et al. (2015a) \\
\hline \multirow[t]{2}{*}{ Litopenaeus stylirostris } & $\mathrm{AEP}(\mathrm{SIN})$ & $0.30^{\mathrm{D}}(1998-1999) \mathrm{M}$ & Ruelas-Inzunza et al. (2004) \\
\hline & AEP (SIN) & $0.09^{\mathrm{W}}(1998-1999) \mathrm{M}$ & Ruelas-Inzunza et al. (2011a) \\
\hline Litopenaeus setiferus & LTM (CAM) & ND (2011) M & Benítez et al. (2012) \\
\hline \multirow[t]{2}{*}{ Penaeus aztecus } & LAV (VER) & $0.008(2003) \mathrm{M}$ & Guentzel et al. (2007) \\
\hline & LAV (VER) & $0.03^{\mathrm{W}}(2005) \mathrm{M}$ & Guentzel et al. (2007) \\
\hline Farfantepenaeus & AEP (SIN) & $0.06^{\mathrm{W}}(1998-1999) \mathrm{M}$ & Ruelas-Inzunza et al. (2011a) \\
\hline brevirostris & $\mathrm{AEP}(\mathrm{SIN})$ & $0.21^{\mathrm{D}}(1998-1999) \mathrm{M}$ & Ruelas-Inzunza et al. (2004) \\
\hline Farfantepenaeus & AEP (SIN) & $0.04^{\mathrm{W}}(1998-1999) \mathrm{M}$ & Ruelas-Inzunza et al. (2011a) \\
\hline californiensis & AEP (SIN) & $0.13^{\mathrm{D}}(1998-1999) \mathrm{M}$ & Ruelas-Inzunza et al. (2004) \\
\hline \multirow[t]{2}{*}{ Xiphopenaeus kroyeri } & AEP (SIN) & $0.04^{\mathrm{W}}(1998-1999) \mathrm{M}$ & Ruelas-Inzunza et al. (2011a) \\
\hline & AEP (SIN) & $0.13^{\mathrm{D}}(1998-1999) \mathrm{M}$ & Ruelas-Inzunza et al. (2004) \\
\hline Palaemonetes & $\mathrm{BBCN}(\mathrm{SON})$ & $0.40^{\mathrm{D}}(1998-2000) \mathrm{T}$ & García-Hernández et al. (2001) \\
\hline Paludosus & $\operatorname{CSC}(\mathrm{SON})$ & $0.60^{\mathrm{D}}(1998-2000) \mathrm{T}$ & García-Hernández et al. (2001) \\
\hline Callinectes arcuatus & UR (SIN) & $0.13^{\mathrm{D}}(2006) \mathrm{T}$ & Jara-Marini et al. (2012) \\
\hline Callinectes rathbunae & LAV (VER) & $0.02^{\mathrm{W}}(2012) \mathrm{M}$ & Guentzel et al. (2007) \\
\hline \multirow[t]{13}{*}{ Callinectes bellicosus } & $\mathrm{BCY}(\mathrm{SON})$ & $0.20^{\mathrm{W}}(2012) \mathrm{M}$ & García-Hernández et al. (2015) \\
\hline & $\mathrm{BST}(\mathrm{SON})$ & $0.26^{\mathrm{W}}(2012) \mathrm{M}$ & García-Hernández et al. (2015) \\
\hline & BKK (SON) & $0.15^{\mathrm{W}}(2012) \mathrm{M}$ & García-Hernández et al. (2015) \\
\hline & GYEMP (SON) & $0.08^{\mathrm{W}}(2012) \mathrm{M}$ & García-Hernández et al. (2015) \\
\hline & BGMS (SON) & $0.09^{\mathrm{W}}(2012) \mathrm{M}$ & García-Hernández et al. (2015) \\
\hline & $\mathrm{BLB}(\mathrm{SON})$ & $0.05^{\mathrm{W}}(2012) \mathrm{M}$ & García-Hernández et al. (2015) \\
\hline & LTB (SON) & $0.10^{\mathrm{W}}(2012) \mathrm{M}$ & García-Hernández et al. (2015) \\
\hline & BYV (SON) & $0.12^{\mathrm{W}}(2012) \mathrm{M}$ & García-Hernández et al. (2015) \\
\hline & $\mathrm{BAP}(\mathrm{SON})$ & $0.19^{\mathrm{W}}(2012) \mathrm{M}$ & García-Hernández et al. (2015) \\
\hline & INM (SIN) & $0.20^{\mathrm{W}}(2012) \mathrm{M}$ & García-Hernández et al. (2015) \\
\hline & BOH (SIN) & $0.14^{\mathrm{W}}(2012) \mathrm{M}$ & García-Hernández et al. (2015) \\
\hline & SMR (SIN) & $0.09^{\mathrm{W}}(2012) \mathrm{M}$ & García-Hernández et al. (2015) \\
\hline & $\mathrm{AEP}(\mathrm{SIN})$ & $0.13^{\mathrm{W}}(2012) \mathrm{M}$ & García-Hernández et al. (2015) \\
\hline Procambarus clarkii & RMYR (SON) & $0.05^{\mathrm{D}}(1998-200) \mathrm{T}$ & García-Hernández et al. (2001) \\
\hline \multicolumn{4}{|l|}{ Fish } \\
\hline \multirow[t]{2}{*}{ Opisthonema libertate } & BGY (SON) & $0.20^{\mathrm{D}}(1998-1999) \mathrm{M}$ & Ruelas-Inzunza \& Páez-Ozuna (2005) \\
\hline & BGY (SON) & $0.06^{\mathrm{W}}(1998) \mathrm{M}$ & Ruelas-Inzunza et al. (2011a) \\
\hline \multirow[t]{2}{*}{ Paralichthys woolmani } & $\mathrm{BTBP}(\mathrm{SON})$ & $0.68^{\mathrm{W}}(2004) \mathrm{M}$ & Ruelas-Inzunza et al. (2008) \\
\hline & $\mathrm{BTBP}(\mathrm{SON})$ & $0.21^{\mathrm{W}}(2004) \mathrm{M}$ & Ruelas-Inzunza et al. (2011a) \\
\hline
\end{tabular}


continuation

\begin{tabular}{|c|c|c|c|}
\hline Species & Place & $\mathrm{Hg}$ & Reference \\
\hline \multirow[t]{2}{*}{ Cyclopsetta querna } & CGRR (GRO) & $0.21^{\mathrm{D}}(2011) \mathrm{M}$ & Spanopoulos-Zarco et al. (2014) \\
\hline & CSNL (SIN) & $0.39^{\mathrm{D}}(2011) \mathrm{M}$ & Ruelas-Inzunza et al. (2012) \\
\hline Ancylopsetta dendritica & CGRR (GRO) & $0.26^{\mathrm{D}}(2011) \mathrm{M}$ & Spanopoulos-Zarco et al. (2014) \\
\hline Achirus mazatlanus & LBN (JAL) & $0.29^{\mathrm{D}}(2014) \mathrm{M}$ & Aguilar-Betancourt et al. (2016) \\
\hline Bagre marina & LAV (VER) & $0.29^{\mathrm{W}}(2005) \mathrm{M}$ & Guentzel et al. (2007) \\
\hline Cathorops fuerthii & BGY (SON) & $0.45^{\mathrm{D}}(1998-1999) \mathrm{M}$ & Ruelas-Inzunza \& Páez-Ozuna (2005) \\
\hline Cathorops fuerthii & BGY (SON) & $0.14^{\mathrm{W}}(1998) \mathrm{M}$ & Ruelas-Inzunza et al. (2011a) \\
\hline Galeichthys peruvianus & AEP (SIN) & $1.58^{\mathrm{D}}(1998-1999) \mathrm{M}$ & Ruelas-Inzunza \& Páez-Ozuna (2005) \\
\hline Arius platypogon & $\mathrm{BTBP}(\mathrm{SON})$ & $0.29^{\mathrm{W}}(2004) \mathrm{M}$ & Ruelas-Inzunza et al. (2011a) \\
\hline Sciades guatemalensis & LBN (JAL) & $1.43^{\mathrm{D}}(2014) \mathrm{M}$ & Aguilar-Betancourt et al. (2016) \\
\hline Arius felis & LAV (VER) & $0.27^{\mathrm{W}}(2000-2003) \mathrm{M}$ & Elliott et al. (2015) \\
\hline Arius sp. & ESBL (NAY) & $0.20^{\mathrm{W}}(2000-2003) \mathrm{M}$ & Elliott et al. (2015) \\
\hline Ictalurus furcatus & PLL $(\mathrm{CHH})$ & $0.08^{\mathrm{W}}(2001-2012) \mathrm{M}$ & Luna-Porres et al. (2014) \\
\hline Pterygoplichthys pardalis & RUSMT (TAB) & $0.45^{\mathrm{D}}(\mathrm{N} / \mathrm{E}) \mathrm{M}$ & Maldonado-Enríquez et al. (2015) \\
\hline \multirow[t]{2}{*}{ Eugerris plumeri } & LAV (VER) & $0.35^{\mathrm{W}}(2002) \mathrm{M}$ & Guentzel et al. (2007) \\
\hline & LAV (VER) & $0.30^{\mathrm{W}}(2005) \mathrm{M}$ & Guentzel et al. (2007) \\
\hline \multirow[t]{2}{*}{ Eugerris axillaris } & UR (SIN) & $0.70^{\mathrm{D}}(2012) \mathrm{M}$ & Frías-Espericueta et al. (2016) \\
\hline & UR (SIN) & $0.48^{\mathrm{D}}(2013) \mathrm{M}$ & Frías-Espericueta et al. (2016) \\
\hline \multirow[t]{4}{*}{ Gerres cinereus } & UR (SIN) & $0.15^{\mathrm{D}}(2006) \mathrm{M}$ & Jara-Marini et al. (2012) \\
\hline & TCPN (SIN) & $0.29^{\mathrm{W}}(2005) \mathrm{M}$ & Ruelas-Inzunza et al. (2011a) \\
\hline & ERCZ (VER) & $0.09^{\mathrm{W}}(2005) \mathrm{M}$ & Ruelas-Inzunza et al. (2011a) \\
\hline & ERCZ (VER) & $0.19^{\mathrm{D}}(2005) \mathrm{M}$ & Ruelas-Inzunza et al. (2009a) \\
\hline \multirow[t]{3}{*}{ Diapterus peruvianus } & $\mathrm{BTBP}(\mathrm{SON})$ & $0.16^{\mathrm{W}}(2004) \mathrm{M}$ & Ruelas-Inzunza et al. (2011a) \\
\hline & CGRR (GRO) & $0.53^{\mathrm{D}}(2011) \mathrm{M}$ & Spanopoulos-Zarco et al. (2014) \\
\hline & CSNL (SIN) & $2.55^{\mathrm{D}}(2011) \mathrm{M}$ & Ruelas-Inzunza et al. (2012) \\
\hline Diapterus brevirostris & LBN (JAL) & $0.36^{\mathrm{D}}(2014) \mathrm{M}$ & Aguilar-Betancourt et al. (2016) \\
\hline \multirow[t]{5}{*}{ Cyprinus carpio } & LCHP (JAL) & $0.39^{\mathrm{W}}(2010) \mathrm{M}$ & Stong et al. (2013) \\
\hline & LCHP (JAL) & $0.35^{\mathrm{W}}(2011) \mathrm{M}$ & Torres et al. (2014) \\
\hline & LCHP (JAL) & $0.10^{\mathrm{W}}(2012) \mathrm{M}$ & Torres et al. (2014) \\
\hline & PVM (MEX) & $\mathrm{ND}^{\mathrm{W}}(2013) \mathrm{M}$ & Morachis-Valdez et al. (2015) \\
\hline & PLL (CHH) & $0.008^{\mathrm{W}}(2011-2012) \mathrm{M}$ & Luna-Porres et al. (2014) \\
\hline Criprynus sp. & LCHP (JAL) & $0.83^{\mathrm{W}}(2007) \mathrm{M}$ & Trasande et al. (2010) \\
\hline \multirow[t]{3}{*}{ Chirostoma sp. } & LCHP (JAL) & $0.11^{\mathrm{W}}(2007) \mathrm{M}$ & Trasande et al. (2010) \\
\hline & LCHP (JAL) & $0.15^{\mathrm{W}}(2011) \mathrm{M}$ & Torres et al. (2014) \\
\hline & LCHP (JAL) & $0.07^{\mathrm{W}}(2012) \mathrm{M}$ & Torres et al. (2014) \\
\hline \multirow[t]{3}{*}{ Gambusia affinis } & RCL (SON) & $0.63^{\mathrm{D}}(1998-2000) \mathrm{T}$ & García-Hernández et al. (2001) \\
\hline & RHD (SON) & $0.32^{\mathrm{D}}(1998-2000) \mathrm{T}$ & García-Hernández et al. (2001) \\
\hline & RMYR (SON) & $0.89^{\mathrm{D}}(1998-2000) \mathrm{T}$ & García-Hernández et al. (2001) \\
\hline Poecilia latipinna & $\mathrm{CSC}(\mathrm{SON})$ & $0.12^{\mathrm{D}}(1998-2000) \mathrm{T}$ & García-Hernández et al. (2001) \\
\hline \multirow[t]{8}{*}{ Mugil cephalus } & $\mathrm{CCP}(\mathrm{SON})$ & $0.04^{\mathrm{D}}(1998-2000) \mathrm{T}$ & García-Hernández et al. (2001) \\
\hline & $\mathrm{BGY}(\mathrm{SON})$ & $0.02^{\mathrm{D}}(1998-1999) \mathrm{M}$ & Ruelas-Inzunza \& Páez-Ozuna (2005) \\
\hline & AEP (SIN) & $0.13^{\mathrm{D}}(1998-1999) \mathrm{M}$ & Ruelas-Inzunza \& Páez-Ozuna (2005) \\
\hline & $\mathrm{UR}(\mathrm{SIN})$ & $0.06^{\mathrm{D}}(2006) \mathrm{T}$ & Jara-Marini et al. (2012) \\
\hline & BGY (SON) & $\mathrm{ND}^{\mathrm{D}}(1998) \mathrm{M}$ & Ruelas-Inzunza et al. (2011a) \\
\hline & BTBP (SON) & $0.01^{\mathrm{W}}(2004) \mathrm{M}$ & Ruelas-Inzunza et al. (2011a) \\
\hline & AEP (SIN) & $0.04^{\mathrm{W}}(1998-1999) \mathrm{M}$ & Ruelas-Inzunza et al. (2011a) \\
\hline & AEP (SIN) & $0.03^{\mathrm{W}}(2004) \mathrm{M}$ & Ruelas-Inzunza et al. (2011a) \\
\hline \multirow[t]{8}{*}{ Mugil curema } & $\mathrm{UR}(\mathrm{SIN})$ & $0.02^{\mathrm{W}}(2003) \mathrm{M}$ & Ruelas-Inzunza et al. (2011a) \\
\hline & SMR (SIN) & $0.03^{\mathrm{W}}(2003) \mathrm{M}$ & Ruelas-Inzunza et al. (2011a) \\
\hline & LAV (VER) & $0.57^{\mathrm{W}}(2003) \mathrm{M}$ & Guentzel et al. (2007) \\
\hline & LCTC (VER) & $0.02^{\mathrm{W}}(2000-2003) \mathrm{M}$ & Elliott et al. (2015) \\
\hline & MTTN (VER) & $0.14^{\mathrm{W}}(2000-2003) \mathrm{M}$ & Elliott et al. (2015) \\
\hline & LAV (VER) & $0.03^{\mathrm{W}}(2000-2003) \mathrm{M}$ & Elliott et al. (2015) \\
\hline & LBN (JAL) & $0.03^{\mathrm{D}}(2014) \mathrm{M}$ & Aguilar-Betancourt et al. (2016) \\
\hline & LCTC (VER) & $0.02^{\mathrm{W}}(2000-2003) \mathrm{M}$ & Elliott et al. (2015) \\
\hline \multirow[t]{4}{*}{ Mugil sp. } & RSTG (NAY) & $0.04^{\mathrm{W}}(2000-2003) \mathrm{M}$ & Elliott et al. (2015) \\
\hline & LBN (JAL) & $0.02^{\mathrm{W}}(2000-2003) \mathrm{M}$ & Elliott et al. (2015) \\
\hline & UR (SIN) & $0.18^{\mathrm{D}}(2012) \mathrm{M}$ & Frías-Espericueta et al. (2016) \\
\hline & UR (SIN) & $0.15^{\mathrm{D}}(2013) \mathrm{M}$ & Frías-Espericueta et al. (2016) \\
\hline Cichlasoma sp. & LBN (JAL) & $0.05^{\mathrm{W}}(2000-2003) \mathrm{M}$ & Elliott et al. (2015) \\
\hline
\end{tabular}


continuation

\begin{tabular}{|c|c|c|c|}
\hline Species & Place & $\mathrm{Hg}$ & Reference \\
\hline \multirow[t]{4}{*}{ Oreochromis sp. } & LCHP (JAL) & $0.04^{\mathrm{W}}(2007) \mathrm{M}$ & Trasande et al. (2010) \\
\hline & LCHP (JAL) & $0.03^{\mathrm{W}}(2011) \mathrm{M}$ & Torres et al. (2014) \\
\hline & LCHP (JAL) & $0.03^{\mathrm{W}}(2012) \mathrm{M}$ & Torres et al. (2014) \\
\hline & CSDR (CHP) & $0.05^{\mathrm{W}}(2000-2003) \mathrm{M}$ & Elliott et al. (2015) \\
\hline Oreochromis sp. & RCZ (VER) & $0.05^{\mathrm{D}}(2005) \mathrm{M}$ & Ruelas-Inzunza et al. (2009a) \\
\hline \multirow[t]{2}{*}{ Oreochromis niloticus } & LCTC (VER) & $0.01^{\mathrm{W}}(2000-2003) \mathrm{M}$ & Elliott et al. (2015) \\
\hline & LAV (VER) & $0.02^{\mathrm{W}}(2000-2003) \mathrm{M}$ & Elliott et al. (2015) \\
\hline \multirow[t]{2}{*}{ Lepomis macrochirus } & RHD (SON) & $0.65^{\mathrm{W}}(2010) \mathrm{M}$ & García-Hernández et al. (2013) \\
\hline & CSC (SON) & $0.69^{\mathrm{D}}(1998-2000) \mathrm{T}$ & García-Hernández et al. (2013) \\
\hline Lepomis cyanellus & PLL (CHH) & $0.07^{\mathrm{W}}(2011-2012) \mathrm{M}$ & Luna-Porres et al. (2014) \\
\hline Archosargus probatocephalus & LAV (VER) & $0.09^{\mathrm{W}}(2002) \mathrm{M}$ & Guentzel et al. (2007) \\
\hline Gobiomorus polylepis & RCZ (VER) & $0.86^{\mathrm{D}}(2006) \mathrm{M}$ & Ruelas-Inzunza et al. (2009a) \\
\hline \multirow[t]{4}{*}{ Centropomus parallelus } & LAV (VER) & $0.15^{\mathrm{W}}(2002) \mathrm{M}$ & Guentzel et al. (2007) \\
\hline & LAV (VER) & $0.18^{\mathrm{W}}(2000-2003) \mathrm{M}$ & Elliott et al. (2015) \\
\hline & MTTN (VER) & $0.29^{\mathrm{W}}(2000-2003) \mathrm{M}$ & Elliott et al. (2015) \\
\hline & TLCP (VER) & $0.13^{\mathrm{W}}(2000-2003) \mathrm{M}$ & Elliott et al. (2015) \\
\hline \multirow[t]{3}{*}{ Centropomus viridis } & ERCZ (VER) & $0.26^{\mathrm{W}}(2005) \mathrm{M}$ & Ruelas-Inzunza et al. (2011a) \\
\hline & ERCZ (VER) & $0.68^{\mathrm{D}}(2005) \mathrm{M}$ & Ruelas-Inzunza et al. (2009a) \\
\hline & ERCZ (VER) & $0.48^{\mathrm{D}}(2006) \mathrm{M}$ & Ruelas-Inzunza et al. (2009a) \\
\hline \multirow[t]{2}{*}{ Centropomus nigrescens } & $\mathrm{BTBP}(\mathrm{SON})$ & $0.56^{\mathrm{D}}(2004) \mathrm{M}$ & Ruelas-Inzunza et al. (2008) \\
\hline & BTBP (SON) & $0.17^{\mathrm{W}}(2004) \mathrm{M}$ & Ruelas-Inzunza et al. (2011a) \\
\hline \multirow[t]{2}{*}{ Centropomus armatus } & $\mathrm{BTBP}(\mathrm{SON})$ & $1.51^{\mathrm{D}}(2004) \mathrm{M}$ & Ruelas-Inzunza et al. (2008) \\
\hline & $\mathrm{BTBP}(\mathrm{SON})$ & $0.44^{\mathrm{W}}(2004) \mathrm{M}$ & Ruelas-Inzunza et al. (2011a) \\
\hline Centropomus medius & SMR (SIN) & $0.82^{\mathrm{D}}(2003) \mathrm{M}$ & Ruelas-Inzunza et al. (2008) \\
\hline Centropomus ensiferus & LAV (VER) & $0.18^{\mathrm{W}}(2005) \mathrm{M}$ & Guentzel et al. (2007) \\
\hline Centropomus sp. & MTCL (NAY) & $0.30^{\mathrm{W}}(2000-2003) \mathrm{M}$ & Elliott et al. (2015) \\
\hline Cynoscion xanthulus & $\mathrm{AEP}(\mathrm{SIN})$ & $0.1^{\mathrm{D}}(1998-1999) \mathrm{M}$ & García-Hernández et al. (2005) \\
\hline \multirow{2}{*}{ Micropogonias ectenes } & CGRR (GRO) & $0.54^{\mathrm{D}}(2011) \mathrm{M}$ & Spanopoulos-Zarco et al. (2014) \\
\hline & CSNL (SIN) & $0.65^{\mathrm{D}}(2011) \mathrm{M}$ & Ruelas-Inzunza et al. (2012) \\
\hline \multirow[t]{2}{*}{ Haemulopsis axillaris } & CGRR (GRO) & $0.51^{\mathrm{D}}(2011) \mathrm{M}$ & Spanopoulos-Zarco et al. (2014) \\
\hline & CSNL (SIN) & $1.69^{\mathrm{D}}(2011) \mathrm{M}$ & Ruelas-Inzunza et al. (2012) \\
\hline \multirow[t]{2}{*}{ Umbrina xanti } & CGRR (GRO) & $0.40^{\mathrm{D}}(2011) \mathrm{M}$ & Spanopoulos-Zarco et al. (2014) \\
\hline & CSNL (SIN) & $0.58^{\mathrm{D}}(2011) \mathrm{M}$ & Ruelas-Inzunza et al. (2012) \\
\hline Pomadasys panamensis & CGRR (GRO) & $0.12^{\mathrm{D}}(2011) \mathrm{M}$ & Spanopoulos-Zarco et al. (2014) \\
\hline Pseudupeneus grandisquamis & CGRR (GRO) & $0.38^{\mathrm{D}}(2011) \mathrm{M}$ & Spanopoulos-Zarco et al. (2014) \\
\hline \multirow{2}{*}{ Hemicaranx leucurus } & CGRR (GRO) & $0.13^{\mathrm{D}}(2011) \mathrm{M}$ & Spanopoulos-Zarco et al. (2014) \\
\hline & CSNL (SIN) & $0.58^{\mathrm{D}}(2011) \mathrm{M}$ & Ruelas-Inzunza et al. (2011) \\
\hline \multirow[t]{5}{*}{ Lutjanus colorado } & $\mathrm{AEP}(\mathrm{SIN})$ & $0.89^{\mathrm{D}}(1998-1999) \mathrm{M}$ & Ruelas-Inzunza \& Páez-Ozuna (2005) \\
\hline & URTCP (SIN) & $0.53^{\mathrm{D}}(2003-2004) \mathrm{M}$ & Ruelas-Inzunza et al. (2008) \\
\hline & UR (SIN) & $0.10^{\mathrm{W}}(2003) \mathrm{M}$ & Ruelas-Inzunza et al. (2011a) \\
\hline & AEP (SIN) & $0.26^{\mathrm{W}}(1998-1999) \mathrm{M}$ & Ruelas-Inzunza et al. (2011a) \\
\hline & UR (SIN) & $0.18^{\mathrm{W}}(2003) \mathrm{M}$ & Ruelas-Inzunza et al. (2011b) \\
\hline Lutjanus argentiventris & UR (SIN) & $0.19^{\mathrm{D}}(2006) \mathrm{T}$ & Jara-Marini et al. (2012) \\
\hline \multirow[t]{3}{*}{ Caranx caninus } & $\mathrm{BTBP}(\mathrm{SON})$ & $3.32^{\mathrm{D}}(2004) \mathrm{M}$ & Ruelas-Inzunza et al. (2008) \\
\hline & $\mathrm{BTBP}(\mathrm{SON})$ & $1.0^{\mathrm{W}}(2004) \mathrm{M}$ & Ruelas-Inzunza et al. (2011a) \\
\hline & BTBP (SON) & $0.2^{\mathrm{W}}(2004) \mathrm{M}$ & Ruelas-Inzunza et al. (2011b) \\
\hline Polydactylus approximans & CSNL (SIN) & $1.72^{\mathrm{D}}(2011) \mathrm{M}$ & Ruelas-Inzunza et al. (2012) \\
\hline Scorpaena sp. & CSNL (SIN) & $1.72^{\mathrm{D}}(2011) \mathrm{M}$ & Ruelas-Inzunza et al. (2012) \\
\hline Oligoplites saurus & $\mathrm{BTBP}(\mathrm{SON})$ & $1.74^{\mathrm{D}}(2004) \mathrm{M}$ & Ruelas-Inzunza et al. (2008) \\
\hline Isopisthus remifer & CSNL (SIN) & $1.77^{\mathrm{D}}(2011) \mathrm{M}$ & Ruelas-Inzunza et al. (2012) \\
\hline \multirow[t]{2}{*}{ Trachinotus kennedyi } & CGRR (GRO) & $0.11^{\mathrm{D}}(2011) \mathrm{M}$ & Spanopoulos-Zarco et al. (2014) \\
\hline & CSNL (SIN) & $0.10^{\mathrm{D}}(2011) \mathrm{M}$ & Ruelas-Inzunza et al. (2012) \\
\hline \multirow[t]{3}{*}{ Seriola lalandei } & BGY (SON) & $0.76^{\mathrm{D}}(1998-1999) \mathrm{M}$ & Ruelas-Inzunza \& Páez-Ozuna (2005) \\
\hline & GCFR (N/A) & $0.07^{\mathrm{W}}(2003-2004) \mathrm{M}$ & García-Hernández et al. (2007) \\
\hline & BGY (SON) & $0.23^{\mathrm{W}}(1998) \mathrm{M}$ & Ruelas-Inzunza et al. (2011a) \\
\hline Thunnus albacares & GCFR (N/A) & $0.03^{\mathrm{W}}(2003-2004) \mathrm{M}$ & García-Hernández et al. (2007) \\
\hline Coryphaena hippurus & GCFR (N/A) & $0.05^{\mathrm{W}}(2003-2004) \mathrm{M}$ & García-Hernández et al. (2007) \\
\hline Makaira mazara & GCFR (N/A) & $0.36^{\mathrm{W}}(2003-2004) \mathrm{M}$ & García-Hernández et al. (2007) \\
\hline Istiophorus platypterus & GCFR (N/A) & $0.40^{\mathrm{W}}(2003-2004) \mathrm{M}$ & García-Hernández et al. (2007) \\
\hline Acanthocybium solandri & GCFR (N/A) & $0.15^{\mathrm{W}}(2003-2004) \mathrm{M}$ & García-Hernández et al. (2007) \\
\hline Tetrapturus audax & GCFR (N/A) & $0.14^{\mathrm{W}}(2003-2004) \mathrm{M}$ & García-Hernández et al. (2007) \\
\hline
\end{tabular}


continuation

\begin{tabular}{|c|c|c|c|}
\hline Species & Place & $\mathrm{Hg}$ & Reference \\
\hline \multicolumn{4}{|l|}{ Sharks and rays } \\
\hline \multirow{2}{*}{ Carcharhinus limbatus } & PVCZ (VER) & $3.33^{\mathrm{W}}(1994-1995) \mathrm{M}$ & Núñez-Noriega (2005) \\
\hline & GCFR (N/A) & $0.51^{\mathrm{W}}(2003-2004) \mathrm{M}$ & García-Hernández et al. (2007) \\
\hline \multirow[t]{2}{*}{ Carcharhinus leucas } & AEP (SIN) & $0.20^{\mathrm{D}}(1998-1999) \mathrm{M}$ & Ruelas-Inzunza \& Páez-Ozuna (2005) \\
\hline & AEP (SIN) & $0.62^{\mathrm{W}}(2003) \mathrm{M}$ & Ruelas-Inzunza et al. (2011b) \\
\hline \multirow[t]{2}{*}{ Carcharhinus falciformis } & GCFR (N/A) & $0.30^{\mathrm{W}}(2003-2004) \mathrm{M}$ & García-Hernández et al. (2007) \\
\hline & PDBC (BCS) & $3.40^{\mathrm{D}}(2001-2005) \mathrm{M}$ & Maz-Courrau et al. (2012) \\
\hline \multirow[t]{4}{*}{ Sphyrna lewini } & $\mathrm{AEP}(\mathrm{SIN})$ & $0.49^{\mathrm{D}}(1998-1999) \mathrm{M}$ & Ruelas-Inzunza \& Páez-Ozuna (2005) \\
\hline & GCFR (N/A) & $1.08^{\mathrm{W}}(2003-2004) \mathrm{M}$ & García-Hernández et al. (2007) \\
\hline & CSPT (SIN) & $0.81^{\mathrm{W}}(2009-2010) \mathrm{M}$ & Hurtado-Banda et al. (2012) \\
\hline & TCPN (SIN) & $0.63^{\mathrm{W}}(2011-2012) \mathrm{M}$ & Bergés-Tiznado et al. (2015) \\
\hline \multirow[t]{2}{*}{ Sphyrna zygaena } & GCFR (N/A) & $8.25^{\mathrm{W}}(2003-2004) \mathrm{M}$ & García-Hernández et al. (2007) \\
\hline & PDBC (BCS) & $0.98^{\mathrm{D}}(2001-2005) \mathrm{M}$ & Maz-Courrau et al. (2012) \\
\hline \multirow[t]{3}{*}{ Prionace glauca } & CBCF (BSC) & $1.39^{\mathrm{W}}(2005-2006)$ & Escobar-Sánchez et al. (2011) \\
\hline & PDBC (BCS) & $1.96^{\mathrm{D}}(2001-2005) \mathrm{M}$ & Maz-Courrau et al. (2012) \\
\hline & PBLCH (BCS) & $1.03^{\mathrm{W}}(2011) \mathrm{M}$ & Barrera-García et al. (2012) \\
\hline \multirow[t]{2}{*}{ Isurus oxyrinchus } & PDBC (BCS) & $1.05^{\mathrm{D}}(2001-2005) \mathrm{M}$ & Maz-Courrau et al. (2012) \\
\hline & IMGD (BCS) & $0.39 \mathrm{w}(2008) \mathrm{M}$ & Vélez-Alavez et al. (2013) \\
\hline Squatina californica & PPG (BCS) & $0.24^{\mathrm{W}}(2012-2013) \mathrm{M}$ & Escobar-Sánchez et al. (2016) \\
\hline Rhizoprionodon terraenove & PVCZ (VER) & $0.76^{\mathrm{W}}(1994-1995) \mathrm{M}$ & Núñez-Noriega (2005) \\
\hline \multirow[t]{2}{*}{ Rhizoprionodon longurio } & GCFR (N/A) & $1.3^{\mathrm{W}}(2003-2004) \mathrm{M}$ & García-Hernández et al. (2007) \\
\hline & $\mathrm{BKN}(\mathrm{SON})$ & $0.91^{\mathrm{W}}(2009-2010) \mathrm{M}$ & Hurtado-Banda et al. (2012) \\
\hline Nasolamia velox & GCFR (N/A) & $1.02^{\mathrm{W}}(2003-2004) \mathrm{M}$ & García-Hernández et al. (2007) \\
\hline \multirow{3}{*}{$\begin{array}{l}\text { Triakis semifasciata } \\
\text { Dasyatis dipterura }\end{array}$} & GCFR (N/A) & $0.08^{\mathrm{W}}(2003-2004) \mathrm{M}$ & García-Hernández et al. (2007) \\
\hline & $\mathrm{BCHY}(\mathrm{SON})$ & $0.946^{\mathrm{D}}(2012) \mathrm{M}$ & Ruelas-Inzunza et al. (2013) \\
\hline & PRY (NAY) & $2.84^{\mathrm{D}}(2012) \mathrm{M}$ & Ruelas-Inzunza et al. (2013) \\
\hline Dasyatis longus & GCFR (N/A) & $0.71^{\mathrm{W}}(2003-2004) \mathrm{M}$ & García-Hernández et al. (2007) \\
\hline Dasyatis brevis & GCFR (N/A) & $0.45^{\mathrm{W}}(2003-2004) \mathrm{M}$ & García-Hernández et al. (2007) \\
\hline Dasyatis longa & PRY (NAY) & $4.465^{\mathrm{D}}(2012) \mathrm{M}$ & Ruelas-Inzunza et al. (2013) \\
\hline \multirow[t]{2}{*}{ Rhinobatos productus } & GCFR (N/A) & $0.31^{\mathrm{W}}(2003-2004) \mathrm{M}$ & García-Hernández et al. (2007) \\
\hline & PPG (BCS) & $0.89^{\mathrm{D}}(2012) \mathrm{M}$ & Ruelas-Inzunza et al. (2013) \\
\hline \multirow[t]{2}{*}{ Rhinoptera steindachnerii } & GCFR (N/A) & $0.43^{\mathrm{W}}(2003-2004) \mathrm{M}$ & García-Hernández et al. (2007) \\
\hline & DTLL (SIN) & $0.37^{\mathrm{W}}(2012) \mathrm{M}$ & Escobar-Sánchez et al. (2014) \\
\hline \multirow[t]{3}{*}{ Narcine entemedor } & PPG (BCS) & $0.101^{\mathrm{D}}(2012) \mathrm{M}$ & Ruelas-Inzunza et al. (2013) \\
\hline & PCHL (BCS) & $1.053^{\mathrm{D}}(2012) \mathrm{M}$ & Ruelas-Inzunza et al. (2013) \\
\hline & GCFR (N/A) & $0.12^{\mathrm{W}}(2003-2004) \mathrm{M}$ & García-Hernández et al. (2007) \\
\hline \multirow[t]{2}{*}{ Zapteryx exasperata } & GCFR (N/A) & $0.11^{\mathrm{W}}(2003-2004) \mathrm{M}$ & García-Hernández et al. (2007) \\
\hline & PCHL (BCS) & $0.898^{\mathrm{D}}(2012) \mathrm{M}$ & Ruelas-Inzunza et al. (2013) \\
\hline \multirow[t]{2}{*}{ Gymnura marmorata } & GCFR (N/A) & $0.14^{\mathrm{W}}(2003-2004) \mathrm{M}$ & García-Hernández et al. (2007) \\
\hline & DTLL (SIN) & $0.706^{\mathrm{D}}(2012) \mathrm{M}$ & Ruelas-Inzunza et al. (2013) \\
\hline Myliobatis californica & GCFR (N/A) & $0.05^{\mathrm{W}}(2003-2004) \mathrm{M}$ & García-Hernández et al. (2007) \\
\hline & $\mathrm{BKN}(\mathrm{SON})$ & $0.33^{\mathrm{W}}(2009-2010) \mathrm{M}$ & Hurtado-Banda et al. (2012) \\
\hline \multirow{2}{*}{ Urolophus halleri } & PPG (BCS) & $1.37^{\mathrm{D}}(2012) \mathrm{M}$ & Ruelas-Inzunza et al. (2013) \\
\hline & PRY (NAY) & $2.33^{\mathrm{D}}(2012) \mathrm{M}$ & Ruelas-Inzunza et al. (2013) \\
\hline Urolophus spp. & PRY (NAY) & $3.713^{\mathrm{D}}(2012) \mathrm{M}$ & Ruelas-Inzunza et al. (2013) \\
\hline \multirow[t]{2}{*}{ Mobula thurstoni } & DTLL (SIN) & $0.09^{\mathrm{W}}(2012) \mathrm{M}$ & Escobar-Sánchez et al. (2014) \\
\hline & SBRB (SON) & $0.20^{\mathrm{W}}(2012) \mathrm{M}$ & Escobar-Sánchez et al. (2014) \\
\hline Mobula munkiana & SBRB (SON) & $0.19^{\mathrm{W}}(2012) \mathrm{M}$ & Escobar-Sánchez et al. (2014) \\
\hline Mobula japanica & SBRB (SON) & $0.14^{\mathrm{W}}(2012) \mathrm{M}$ & Escobar-Sánchez et al. (2014) \\
\hline & DTLL (SIN) & $0.01^{\mathrm{W}}(2012) \mathrm{M}$ & Escobar-Sánchez et al. (2014) \\
\hline Urotrygon chilensis & PCHL (BCS) & $0.52^{\mathrm{D}}(2012) \mathrm{M}$ & Ruelas-Inzunza et al. (2013) \\
\hline Raja velezi & PPG (BCS) & $1.127^{\mathrm{D}}(2012) \mathrm{M}$ & Ruelas-Inzunza et al. (2013) \\
\hline Turtles & & & \\
\hline Chelonia mydas & PDBC (BCS) & $0.021(\mathrm{~N} / \mathrm{E}) \mathrm{M}$ & Kampalath et al. (2006) \\
\hline & LTM (CAM) & $0.37(2009) \mathrm{H}$ & Benítez et al. (2012) \\
\hline Caretta caretta & PDBC (BCS) & $0.026(\mathrm{~N} / \mathrm{E}) \mathrm{M}$ & Kampalath et al. (2006) \\
\hline Lepidochelys olivacea & PDBC (BCS) & $0.05(\mathrm{~N} / \mathrm{E}) \mathrm{M}$ & Kampalath et al. (2006) \\
\hline & PEPA (OAX) & $0.0006(2005-2006) \mathrm{S}$ & Páez-Osuna et al. (2011) \\
\hline & PEPA (OAX) & $0.001(2005-2006) \mathrm{H}$ & Páez-Osuna et al. (2011) \\
\hline Eretmochelys imbrincata & LTM (CAM) & $0.37(2009) \mathrm{H}$ & Benítez et al. (2012) \\
\hline
\end{tabular}


continuation

\begin{tabular}{|c|c|c|c|}
\hline Species & Place & $\mathrm{Hg}$ & Reference \\
\hline \multicolumn{4}{|l|}{ Birds } \\
\hline Phalacrocorax & CSNL (SIN) & $1.49^{\mathrm{D}}(2002) \mathrm{M}$ & Ruelas-Inzunza et al. (2009b) \\
\hline Brasilianus & UR (SIN) & $1.01^{\mathrm{D}}(2006) \mathrm{M}$ & Jara-Marini et al. (2012) \\
\hline Pelecanus occidentalis & CSNL (SIN) & $2.85^{\mathrm{D}}(2002) \mathrm{M}$ & Ruelas-Inzunza et al. (2009b) \\
\hline Anas clypeata & CSNL (SIN) & $0.47^{\mathrm{D}}(2002) \mathrm{M}$ & Ruelas-Inzunza et al. (2009b) \\
\hline Anas discors & CSNL (SIN) & $0.77^{\mathrm{D}}(2002) \mathrm{M}$ & Ruelas-Inzunza et al. (2009b) \\
\hline Aythya affinis & CSNL (SIN) & $1.06^{\mathrm{D}}(2002) \mathrm{M}$ & Ruelas-Inzunza et al. (2009b) \\
\hline Sula leucogaster & SMR (SIN) & $1.12(2010-2012) \mathrm{S}$ & Lerma et al. (2016) \\
\hline \multicolumn{4}{|l|}{ Crocodiles } \\
\hline \multirow[t]{4}{*}{ Crocodylus moreletti } & RCHPT (CAM) & $3.1(2012) \mathrm{B}$ & Trillanes et al. (2014) \\
\hline & LPNCLT (ROO) & 4.3 (2012) B & Trillanes et al. (2014) \\
\hline & RHND (ROO) & $20(2012-2013) \mathrm{S}$ & Buenfil-Rojas et al. (2015) \\
\hline & RHND (ROO) & $0.33(2012-2013) \mathrm{B}$ & Buenfil-Rojas et al. (2015) \\
\hline \multicolumn{4}{|l|}{ Mammals } \\
\hline Tursiops truncatus & LTM (CAMP) & ND (2011) B & Benítez et al. (2012) \\
\hline Stenella longirostris & GCFR (N/A) & $1.2(1993) \mathrm{M}$ & Ruelas-Inzunza et al. (2003) \\
\hline Trichechus manatus & LTM (CAMP) & ND (2011) B & Benítez et al. (2012) \\
\hline Eschrichtius robustus & GCFR (N/A) & $0.14(1999) \mathrm{M}$ & Ruelas-Inzunza et al. (2003) \\
\hline Baleanoptera musculus & GCFR (N/A) & $0.06(2012) \mathrm{B}$ & Ruelas-Inzunza et al. (2014) \\
\hline
\end{tabular}

This genus of fish is one of the most used around the world in aquatic monitoring studies (Waltham et al., 2013), since they are directly associated with sediments, allowing to evaluate the transfer of pollutants from this environmental matrix to the first levels of the trophic chain. Other commercially important fish that have been used in Mexico are snooks, mojarras, and snappers. Other organisms belonging to different trophic levels have also been used for biomonitoring purposes, including crustaceans, sharks, rays, macroalgae (which also have a high commercial value), sea turtles, birds, crocodiles, and mammals.

\section{Mercury in biota}

Table 1 shows the average concentrations of $\mathrm{Hg}$ found in various tissues of aquatic organisms in Mexico. The average values of $\mathrm{Hg}$ in macrophytes were $0.02-0.09 \mu \mathrm{g}$ $\mathrm{g}^{-1} \mathrm{dw}$ (dry weight) for Gracilaria sp., and $0.05 \mu \mathrm{g} \mathrm{g}^{-1}$ $\mathrm{dw}$ for Ulva lactuca, both cases in the Gulf area of California. No references for the presence of $\mathrm{Hg}$ in macrophytes were found in other areas of the country. Similar concentrations have been reported in other areas around the world. Qiu \& Wang (2016) reported concentrations of $0.04 \mu \mathrm{g} \mathrm{g}^{-1} \mathrm{dw}$ in U. fasciata in Daya Bay in southern China. Pereira et al. (2008) reported average $\mathrm{Hg}$ values of $0.04-0.34 \mu \mathrm{g} \mathrm{g}^{-1} \mathrm{dw}$ in Gracilaria sp. in Ria de Aveiro, Portugal. Akcali \& Kucuksezgin (2011) reported a much higher average $\mathrm{Hg}$ (53-72 $\mu \mathrm{g}$ $\mathrm{g}^{-1} \mathrm{dw}$ ) in Ulva sp. found in Turkey's Aegean coast.

Regarding mollusks, its use has increased in the Gulf of California area. We found 15 studies focused on important ecosystems on the Gulf of California's coastal area during 1997-2011 (sampling year) that used the genus Crassostrea species. These studies reported average values of $\mathrm{Hg}$ of $0.05-0.9 \mu \mathrm{g} \mathrm{g}^{-1} \mathrm{dw}$, EL Colorado Lagoon being the site with the highest concentrations $\left(0.54 \mu \mathrm{g} \mathrm{g}^{-1} \mathrm{dw}\right.$ in 2008 and $0.93 \mu \mathrm{g} \mathrm{g}^{-1}$ $\mathrm{dw}$ in an undetermined date), and the Urías Lagoon with the lowest concentrations $\left(0.05 \mu \mathrm{g} \mathrm{g}^{-1} \mathrm{dw}\right.$ in 2006 and $0.17 \mu \mathrm{g} \mathrm{g}^{-1} \mathrm{dw}$ in 2008). It is known that there are difficulties when comparing the levels of metals in mollusks of different species or different geographical areas since the concentrations can vary depending on the metabolism, age, gonadal stage, habitat (freshwater, marine; lagoons, estuaries, mangroves), sampling season, among others. Thus, the comparison of pollution levels between different mollusk species must be made with reservations (Ruelas-Inzunza et al., 2014).

There is little information on the content of $\mathrm{Hg}$ in Crassostrea in other areas of the country. Benítez et al. (2012) reported concentrations $<0.001 \mu \mathrm{g} \mathrm{g}^{-1} \mathrm{dw}$ in Crassostrea virginica collected in the Terminos Lagoon, in Campeche. Aguilar et al. (2012) reported concentrations of $0.5,0.7$, and $1.1 \mu \mathrm{g} \mathrm{g}^{-1} \mathrm{dw}$ in $C$. virginica collected in the Palizada, Candelaria, and Chumpán rivers, in the state of Campeche, respectively. No other studies with Crassostrea were found in other areas of the country during the review period. However, Hicks et al. (1976), in Villanueva \& Botello (1992), reported concentrations of $0.05 \mu \mathrm{g} \mathrm{g}^{-1} \mathrm{dw}$ in $C$. virginica collected in the Terminos Lagoon. Chione bivalves were also used to assess the concentration of $\mathrm{Hg}$ in coastal areas of the Gulf of California, Kuu Kaak Bay, in the state of Sonora, was where the lowest concen- 
Table 2. Average concentration of $\mathrm{Cd}$ and $\mathrm{Pb}\left(\mu \mathrm{g} \mathrm{g}^{-1} \mathrm{dw}, \mathrm{D}\right.$ or ww, W) in different tissues of organisms from various aquatic ecosystems of Mexico. EU: Estero Urías; AEP: Altata-Ensenada del Pabellón; BTS: Bahía Todos Santos; BGY: Bahía Guaymas; EBNT: Estero Banderitas; CSNR: Costa de Sonora; CSNL: Costa de Sinaloa; GNYR: Granjas Nayarit; BBP: Bahía de Bacochibampo; INM: Laguna San Ignacio-Navachiste-Macapule; BCT: Bahía de Cueta; PAGC: Parte alta del Golfo de California; PCHG: Pichiligue; BBCN: Bocana; BLRT: Bahía de Loreto; GYEMP: Guaymas-Empalme LTM: Laguna de Términos; LTB: Laguna El Tobari; LSLQ: San Luquitas; SMR: Santa María- La Reforma; LCL: El Colorado; ERY: Estero El Rey; LCHP: Lago de Chapala; GSC: Golfo de Santa Clara; LHCM: Laguna Huizache-Caimanero; LGM: Laguna Guasimo; LSI: Laguna de San Ignacio; BKK: Bahía de Kuu Kaak; LOL: Laguna Ojo de Liebre; BGMS: Bahía Las Guácimas; LAV: Laguna Alvarado; LYLH: Laguna Yalahau; BBP: Bacochibampo; BTBP: Bahía de Topolobampo; BAP: Laguna Agiabampo; BOH: Bahía de Ohuria; PPCD: Pescadero; BCHC: Bachoco; CSC: Ciénega Santa Clara; BMGD: Bahía Magdalena; LLRL: Los Laureles; CLSNR: Canal Sonora; PLM: Puerto López Mateos; DRC: Delta del Río Colorado; LBN: Laguna Barra de Navidad; SBRB: Santa Bárbara; RPL: Río Palizada; RCHP: Río Chumpán; BTPB: Bahía de Topolobampo; BYV: Laguna de Yavaros; BCMCH: Boca Camichín; BCHM: Bahía Chetumal; BPZ: Bahía La Paz; PLL: Presa Luis León; LSPT: Laguna de San Pedrito; CSDR: Cañón del sumidero; MTCL: Mercado de Tecuala; RCL: Río Colorado; PSLT: Presa el Salto; RCDLR: Río Candelaria; BCY: Bahía La Choya; BGSB: Guasabe; ESBL: Estero de San Blas; LCTC: Lago de Catemaco; ICND: Islas Coronado; BLB: Bahía de Lobos; RMYR: El Mayor; RUSMT: Río Usumacinta; TCPN: Teacapán; PVM: Presa Viejo Mandín; CCP: Cucapa; LSTN: Laguna Ostión; MTTN: Minatitlán; RSTG: Río Santiago; LMCN: Lago Mecoacán; TLCP: Tlacotalpan; URTCP: Urías-Teacapán; PVCZ: Puerto de Veracruz; LTJ: Laguna Tasajera; CSPT: Cospita; CBCF: Baja California; LTMH: Laguna de Tamiahua; PSCLL: Playa La Escobilla; PBLCH: Punta Belcher; NVT: Nuevo Vallarta; PBND: Punta Banda; LSRL: Laguna de Santa Rosalía; LVCH: Verde Camacho; LMXT: Laguna de Mexcaltitlán; PMZ: Puerto de Mazatlán; DTLL: Dautillos; CDOC: El Doctor; RNJ: Río Naranjos; PCHL: Puerto Chale; CARL: Curso Alto de Río Lerma; RCHPT: Río Champotón; CCMP: Costa de Campeche; LPNCLT: Los Petenes-Celestún; RSPSP: Río San Pedro y San Pablo RSPT: Río San Pedrito; RHND: Río Hondo; PAA: Presa Antonio Alzate; CFN: Infiernillo; RLM: Río Lerma; RPJ: Río Pantoja; LSA: Laguna de San Andrés; LTPL: Laguna Tres Palos; LMDG: Laguna Mandinga. SON: Sonora; SIN: Sinaloa; NAY: Nayarit; JAL: Jalisco; COL: Colima; MIC: Michoacán; GRO: Guerrero; OAX: Oaxaca; CHP: Chiapas; CAM: Campeche; ROO: Quintana Roo; YUC: Yucatán; TAB: Tabasco; VER: Veracruz; TAM: Tamaulipas; BCS: Baja California Sur; BCN: Baja California Norte; AGU: Aguascalientes; MEX: México; CMX: Ciudad de México. N/E: not specified; ND: not detect; -: not analized; D: dry weight; W: wet weight; M: muscle; ST: soft tissue; T: whole body; S: blood; B: biopsy; H: eggs.

\begin{tabular}{|c|c|c|c|c|}
\hline Species & Place & $\mathrm{Cd}$ & $\mathrm{Pb}$ & References \\
\hline \multicolumn{5}{|l|}{ Primary producers } \\
\hline Plankton & PAA (MEX) & - & $8^{\mathrm{W}}(1998-1999)$ & Rodríguez et al. (2001) \\
\hline Phytoplankton & $\mathrm{AEP}(\mathrm{SIN})$ & $0.27^{\mathrm{D}}(1998-1999)$ & $23^{\mathrm{D}}(1998-1999)$ & Ruelas-Inzunza \& Páez-Osuna (2006) \\
\hline \multirow[t]{4}{*}{ Ulva lactuca } & INM (SIN) & $1.9^{\mathrm{D}}(2002)$ & $174.8^{\mathrm{D}}(2002)$ & Orduña-Rojas \& Longoria-Espinoza (2006) \\
\hline & BGY (SON) & $0.54^{\mathrm{D}}(1998-1999)$ & $0.35^{\mathrm{D}}(1998-1999)$ & Ruelas-Inzunza \& Páez-Osuna (2006) \\
\hline & LCHT (SIN) & - & $3^{\mathrm{D}}(2002-2003)$ & Soto-Jiménez et al. (2008) \\
\hline & UR (SIN) & . & $3^{\text {D }}(2002-2003)$ & Soto-Jiménez et al. (2008) \\
\hline \multirow[t]{2}{*}{ Gracilaria textorii } & BMGD (BCS) & $3.65^{\mathrm{D}}(2004-2005)$ & $0.83^{\mathrm{D}}(2004-2005)$ & Talavera-Sáenz et al. (2007) \\
\hline & EBNT (BCS) & $3.16^{\mathrm{D}}(2004-2005)$ & $0.9^{\mathrm{D}}(2004-2005)$ & Riosmena-Rodríguez et al. (2010) \\
\hline Gracilaria subsecundata & $\mathrm{AEP}(\mathrm{SIN})$ & $0.18^{\mathrm{D}}(1998-1999)$ & $0.19^{\mathrm{D}}(1998-1999)$ & Ruelas-Inzunza \& Páez-Osuna (2006) \\
\hline \multirow[t]{4}{*}{ Gracilaria vermiculophylla } & BMGD (BCS) & $1.40^{\mathrm{D}}(2004-2005)$ & $0.84^{\mathrm{D}}(2004-2005)$ & Talavera-Sáenz et al. (2007) \\
\hline & EBNT (BCS) & $1.36^{\mathrm{D}}(2004-2005)$ & $0.8^{\mathrm{D}}(2004-2005)$ & Riosmena-Rodríguez et al. (2010) \\
\hline & UR (SIN) & - & $3.9^{\mathrm{D}}(2002-2003)$ & Soto-Jiménez et al. (2008) \\
\hline & LCHT (SIN) & - & $5^{\mathrm{D}}(2002-2003)$ & Soto-Jiménez et al. (2008) \\
\hline \multirow[t]{2}{*}{ Gracilaria sp. } & $\mathrm{AEP}(\mathrm{SIN})$ & $0.23^{\mathrm{D}}(1998-1999)$ & $4.9^{\mathrm{D}}(1998-1999)$ & Ruelas-Inzunza \& Páez-Osuna (2006) \\
\hline & AEP (SIN) & $0.23^{\mathrm{D}}(1999-2000)$ & $4.9^{\mathrm{D}}(1999-2000)$ & Ruelas-Inzunza \& Páez-Osuna 2008 \\
\hline \multirow[t]{3}{*}{ Zostera marina } & LSI (BCS) & $6.2^{\mathrm{D}}(2000)$ & $8.8^{\mathrm{D}}(2000)$ & Macías-Zamora et al. (2008) \\
\hline & LOL (BCS) & $1.6^{\mathrm{D}}(2000)$ & $14.9^{\mathrm{D}}(2000)$ & Macías-Zamora et al. (2008) \\
\hline & BMGD (BCS) & $1.09^{\mathrm{D}}(2004-2005)$ & $1.23^{\mathrm{D}}(2004-2005)$ & Talavera-Sáenz et al. (2007) \\
\hline \multirow[t]{2}{*}{ Ruppia maritima } & EBNT (BCS) & $4.5^{\mathrm{D}}(2004-2005)$ & $2.15^{\mathrm{D}}(2004-2005)$ & Riosmena-Rodríguez et al. (2010) \\
\hline & BMGD (BCS) & $4.52^{\mathrm{D}}(2004-2005)$ & $2.12^{\mathrm{D}}(2004-2005)$ & Talavera-Sáenz et al. (2007) \\
\hline Caulerpa sertularioides & UR (SIN) & $0.14^{\mathrm{D}}(2006)$ & $2.45^{\mathrm{D}}(2006)$ & Jara-Marini et al. (2008) \\
\hline Echhornia crassipes & RLM (MEX) & - & $4.32^{\mathrm{D}}(2006)$ & Tejeda et al. (2010) \\
\hline Hydrocoyle ranunculoides & CARL (MEX) & - & $5.27^{\mathrm{D}}(2010)$ & Zarazúa et al. (2013) \\
\hline Thalassia testudinum & LYLH (YUC) & $0.72^{\mathrm{D}}(2005-2006)$ & & Avelar et al. (2013) \\
\hline Polysiphonia & AEP (SIN) & $0.87^{\mathrm{D}}(1998-1999)$ & 3.1D (1998-1999) & Ruelas-Inzunza \& Páez-Osuna (2006) \\
\hline
\end{tabular}


continuation

\begin{tabular}{|c|c|c|c|c|}
\hline Species & Place & $\mathrm{Cd}$ & $\mathrm{Pb}$ & References \\
\hline \multicolumn{5}{|l|}{ Bivalves } \\
\hline \multirow{22}{*}{ Crassostrea corteziensis } & UR (SIN) & $2.6^{\mathrm{D}}(2003-2004) \mathrm{ST}$ & $11.5^{\mathrm{D}}(2003-2004) \mathrm{ST}$ & Frías-Espericueta et al. (2005) \\
\hline & $\mathrm{UR}(\mathrm{SIN})$ & - & $4.9^{\mathrm{D}}(2002-2003) \mathrm{M}$ & Soto-Jiménez et al. (2008) \\
\hline & AEP (SIN) & $6.47^{\mathrm{D}}(2008) \mathrm{ST}$ & $2.11^{\mathrm{D}}(2008) \mathrm{ST}$ & Páez-Osuna \& Osuna-Martínez (2015) \\
\hline & $\mathrm{BBP}(\mathrm{SON})$ & $3.2^{\mathrm{W}}(2004-2005) \mathrm{ST}$ & $0.5^{\mathrm{W}}(2004-2005) \mathrm{ST}$ & García-Rico et al. (2010) \\
\hline & INM (SIN) & - & $3.72^{\mathrm{D}}(1996) \mathrm{ST}$ & Páez-Osuna et al. (2002) \\
\hline & SMR (SIN) & $9.02^{\mathrm{D}}$ (2008) ST & $1.64^{\mathrm{D}}(2008) \mathrm{ST}$ & Páez-Osuna \& Osuna-Martínez (2015) \\
\hline & $\mathrm{BCT}(\mathrm{SIN})$ & - & $7.11^{\mathrm{D}}(1996) \mathrm{S}$ & Páez-Osuna et al. (2002) \\
\hline & BCT (SIN) & $4.27^{\mathrm{D}}(2008) \mathrm{ST}$ & $0.96^{\mathrm{D}}(2008) \mathrm{ST}$ & Páez-Osuna \& Osuna-Martínez (2015) \\
\hline & $\mathrm{AEP}(\mathrm{SIN})$ & $7.2^{\mathrm{D}}(1999-2000) \mathrm{ST}$ & $3.4^{\mathrm{D}}(1999-2000) \mathrm{ST}$ & Ruelas-Inzunza \& Páez-Osuna 2008 \\
\hline & UR (SIN) & $1.45^{\mathrm{D}}(2008) \mathrm{ST}$ & $1.36^{\mathrm{D}}(2008) \mathrm{ST}$ & Páez-Osuna \& Osuna-Martínez (2015) \\
\hline & SMR (SIN) & - & $5.32^{\mathrm{D}}(1996) \mathrm{ST}$ & Páez-Osuna et al. (2002) \\
\hline & AEP (SIN) & - & $7.62^{\mathrm{D}}$ (1996) ST & Páez-Osuna et al. (2002) \\
\hline & PMZ (SIN) & - & $7.42^{\mathrm{D}}$ (1996) ST & Páez-Osuna et al. (2002) \\
\hline & LHCM (SIN) & - & $5.22^{\mathrm{D}}$ (1996) ST & Páez-Osuna et al. (2002) \\
\hline & TPAB (NAY) & - & $3.63^{\mathrm{D}}(1996) \mathrm{ST}$ & Páez-Osuna et al. (2002) \\
\hline & LMXT (NAY) & - & $5.10^{\mathrm{D}}(1996) \mathrm{ST}$ & Páez-Osuna et al. (2002) \\
\hline & LSCL (NAY) & - & $2.33^{\mathrm{D}}(1996) \mathrm{ST}$ & Páez-Osuna et al. (2002) \\
\hline & NVT (JAL) & - & $4.72^{\mathrm{D}}(1996) \mathrm{ST}$ & Páez-Osuna et al. (2002) \\
\hline & LBN (JAL) & - & 4.83D (1996) ST & Páez-Osuna et al. (2002) \\
\hline & INM (SIN) & $6.4^{\mathrm{D}}$ (2006-2007) ST & $4.8^{\mathrm{D}}$ (2006-2007) ST & Frías-Espericueta et al. (2009) \\
\hline & SMR (SIN) & $5.8^{\mathrm{D}}$ (2006-2007) ST & $5.3^{\mathrm{D}}(2006-2007) \mathrm{ST}$ & Frías-Espericueta et al. (2009) \\
\hline & AEP (SIN) & $5.01^{\mathrm{D}}(2006-2007)$ & $5.7^{\mathrm{D}}(2006-2007) \mathrm{ST}$ & Frías-Espericueta et al. (2009) \\
\hline \multirow{7}{*}{ Crassostrea gigas } & CSNR (SON) & $0.76^{\mathrm{W}}$ (1997) ST & $0.5^{\mathrm{W}}(1997) \mathrm{ST}$ & García-Rico \& Ramos-Ruiz (2001) \\
\hline & LTB (SON) & $0.38^{\mathrm{D}}(2009) \mathrm{ST}$ & $2.35^{\mathrm{D}}$ (2009) ST & Jara-Marini et al. (2013) \\
\hline & AEP (SIN) & $12.1^{\mathrm{D}}(\mathrm{N} / \mathrm{E}) \mathrm{ST}$ & $1.42^{\mathrm{D}}(\mathrm{N} / \mathrm{E}) \mathrm{ST}$ & Osuna-Martínez et al. (2011) \\
\hline & BCT (SIN) & $8.6^{\mathrm{D}}(\mathrm{N} / \mathrm{E}) \mathrm{ST}$ & $1.11^{\mathrm{D}}(\mathrm{N} / \mathrm{E}) \mathrm{ST}$ & Osuna-Martínez et al. (2011) \\
\hline & SMR (SIN) & $11.55^{\mathrm{D}}(\mathrm{N} / \mathrm{E}) \mathrm{ST}$ & $0.92^{\mathrm{D}}(\mathrm{N} / \mathrm{E}) \mathrm{ST}$ & Osuna-Martínez et al. (2011) \\
\hline & CSNR (SON) & $5.5^{\mathrm{D}}(\mathrm{N} / \mathrm{E}) \mathrm{ST}$ & $1.3^{\mathrm{D}}(\mathrm{N} / \mathrm{E}) \mathrm{ST}$ & Vázquez -Boucard et al. (2014) \\
\hline & CSNL (SIN) & $3.4^{\mathrm{D}}(\mathrm{N} / \mathrm{E}) \mathrm{ST}$ & $2.2^{\mathrm{D}}(\mathrm{N} / \mathrm{E}) \mathrm{ST}$ & Vázquez -Boucard et al. (2014) \\
\hline \multirow[t]{10}{*}{ Crassostrea virginica } & LSA (TAM) & $2.27^{\mathrm{D}}(2000) \mathrm{ST}$ & $0.802^{\mathrm{D}}(2000) \mathrm{ST}$ & Vázquez-Sauceda et al. (2005) \\
\hline & LTM (CAM) & $2.55^{\mathrm{D}}(2002) \mathrm{ST}$ & - & Gold-Bouchot et al. (2007) \\
\hline & LMDG (VER) & $2.2^{\mathrm{D}}(2003-2004) \mathrm{ST}$ & $5.84^{\mathrm{D}}(2003-2004) \mathrm{ST}$ & Guzmán-García et al. (2009) \\
\hline & LTMH (VER) & $11.8^{\mathrm{D}}(2004) \mathrm{ST}$ & $0.5^{\mathrm{D}}(2004) \mathrm{ST}$ & Lango-Reynoso et al. (2010) \\
\hline & LSA (TAM) & $2.27^{\mathrm{D}}(2000) \mathrm{ST}$ & $0.802^{\mathrm{D}}(2000) \mathrm{ST}$ & Vázquez-Sauceda et al. (2011) \\
\hline & LTM (CAM) & $0.0295^{\mathrm{W}}(\mathrm{N} / \mathrm{E}) \mathrm{ST}$ & $0.1445^{\mathrm{W}}(\mathrm{N} / \mathrm{E}) \mathrm{ST}$ & Aguilar-Ucán et al. (2014) \\
\hline & $\mathrm{LMCN}(\mathrm{TAB})$ & $42.23^{\mathrm{D}}$ (2007) ST & $189.78^{\mathrm{D}}$ (2007) ST & Castañeda-Chávez et al. (2014) \\
\hline & RPJ (TAB) & $2.33^{\mathrm{D}}(2011) \mathrm{ST}$ & $0.8^{\mathrm{D}}(2011) \mathrm{ST}$ & Castañeda-Chávez et al. (2014) \\
\hline & RPL (CAM) & $3^{\mathrm{D}}(\mathrm{N} / \mathrm{E}) \mathrm{ST}$ & - & Aguilar et al. (2012) \\
\hline & RCHP (CAM) & $\left.3.2^{\mathrm{D}} \mathrm{N} / \mathrm{E}\right) \mathrm{ST}$ & - & Aguilar et al. (2012) \\
\hline Crassostrea iridescens & PMZ (SIN) & $2.3^{\mathrm{D}}(1995-1996) \mathrm{ST}$ & $2.3^{\mathrm{D}}(1995-1996) \mathrm{ST}$ & Soto-Jiménez et al. (2001) \\
\hline \multirow[t]{2}{*}{ Megapitaria squalida } & BKK (SON) & $0.4^{\mathrm{W}}(2003) \mathrm{ST}$ & $0.001^{\mathrm{W}}(2003) \mathrm{ST}$ & García-Hernández et al. (2005) \\
\hline & $\mathrm{BPZ}(\mathrm{BCS})$ & $5.57^{\mathrm{D}}(2003) \mathrm{ST}$ & $2.5^{\mathrm{D}}(2003) \mathrm{ST}$ & Méndez et al. (2006) \\
\hline
\end{tabular}


continuation

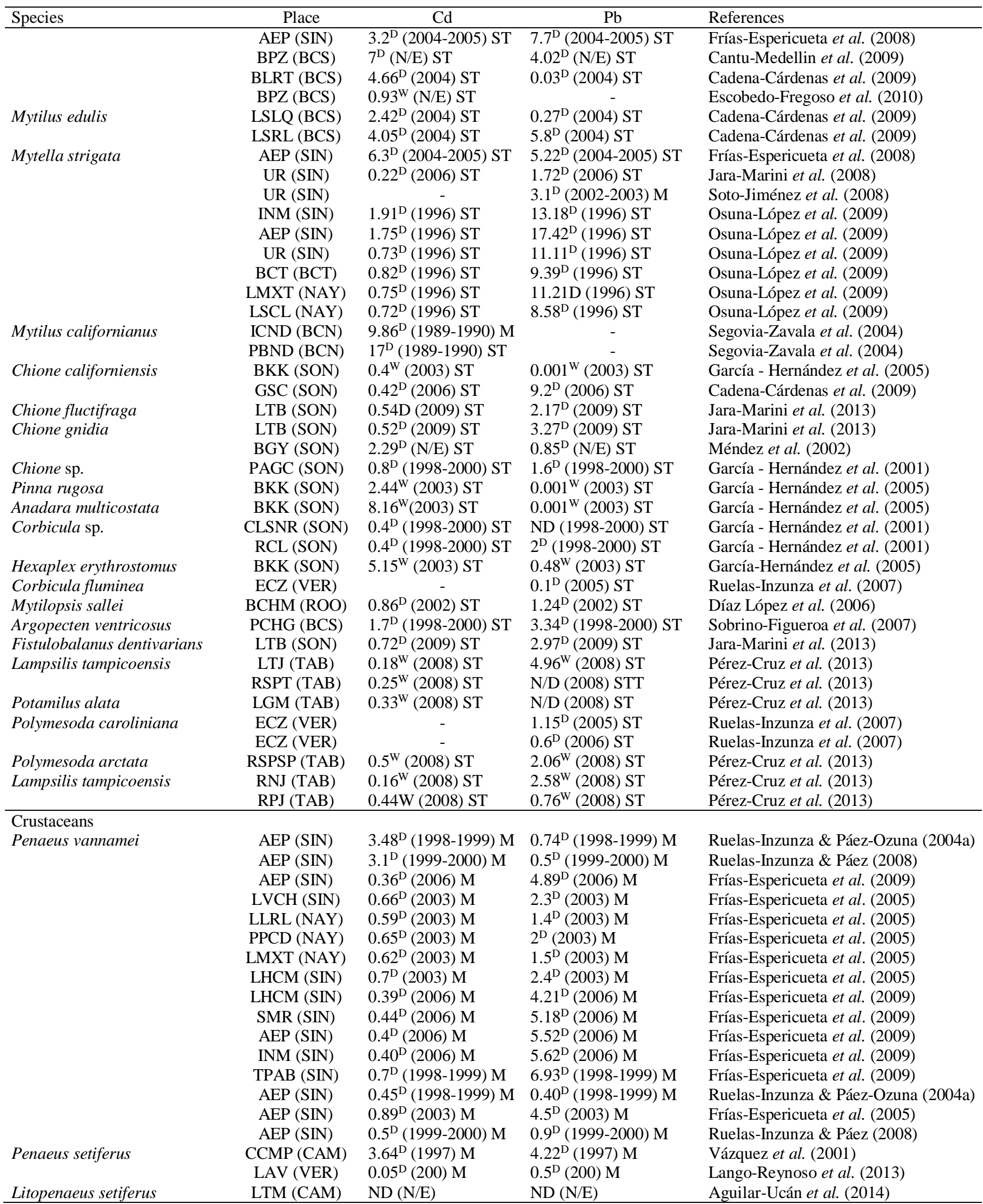


continuation

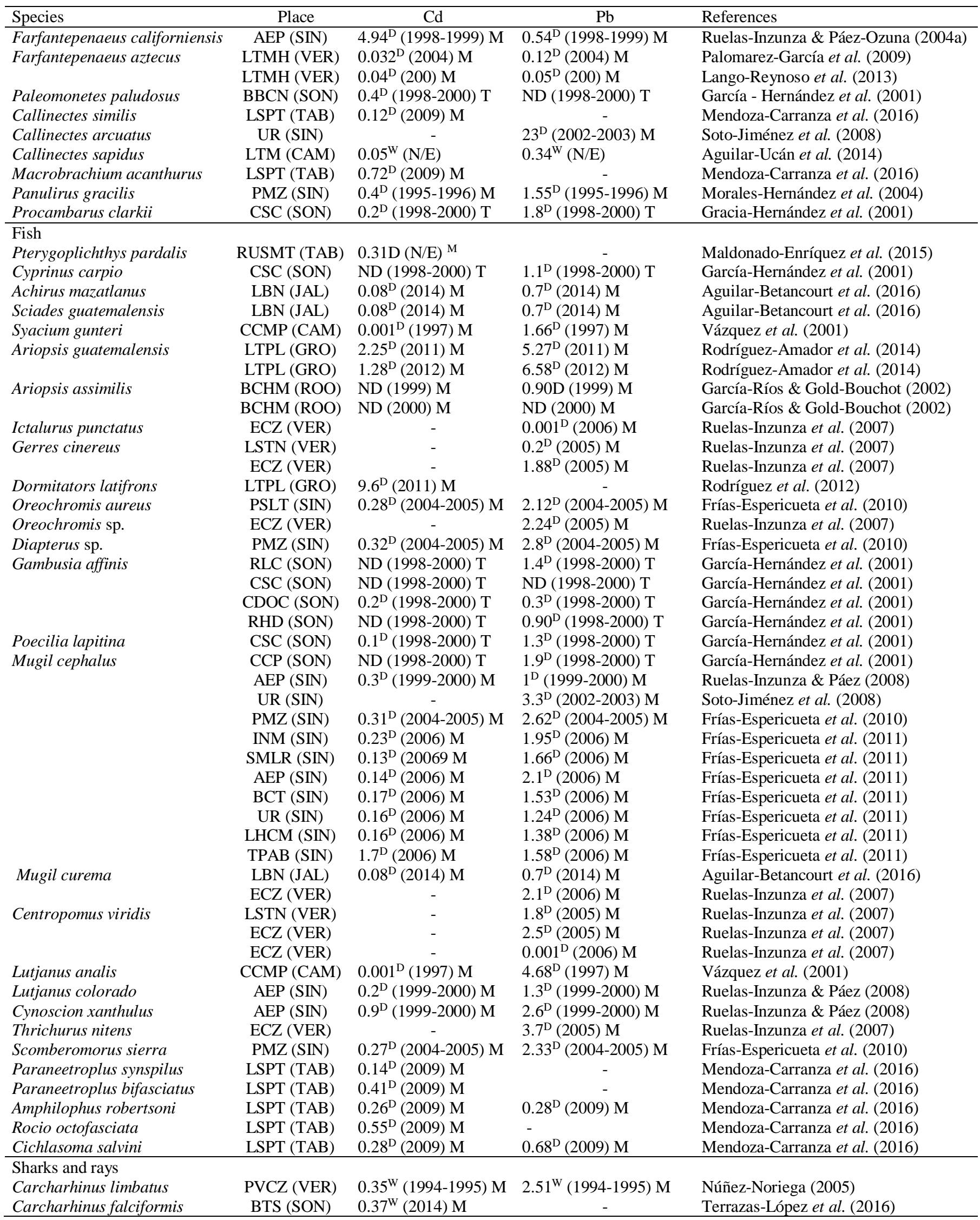


continuation

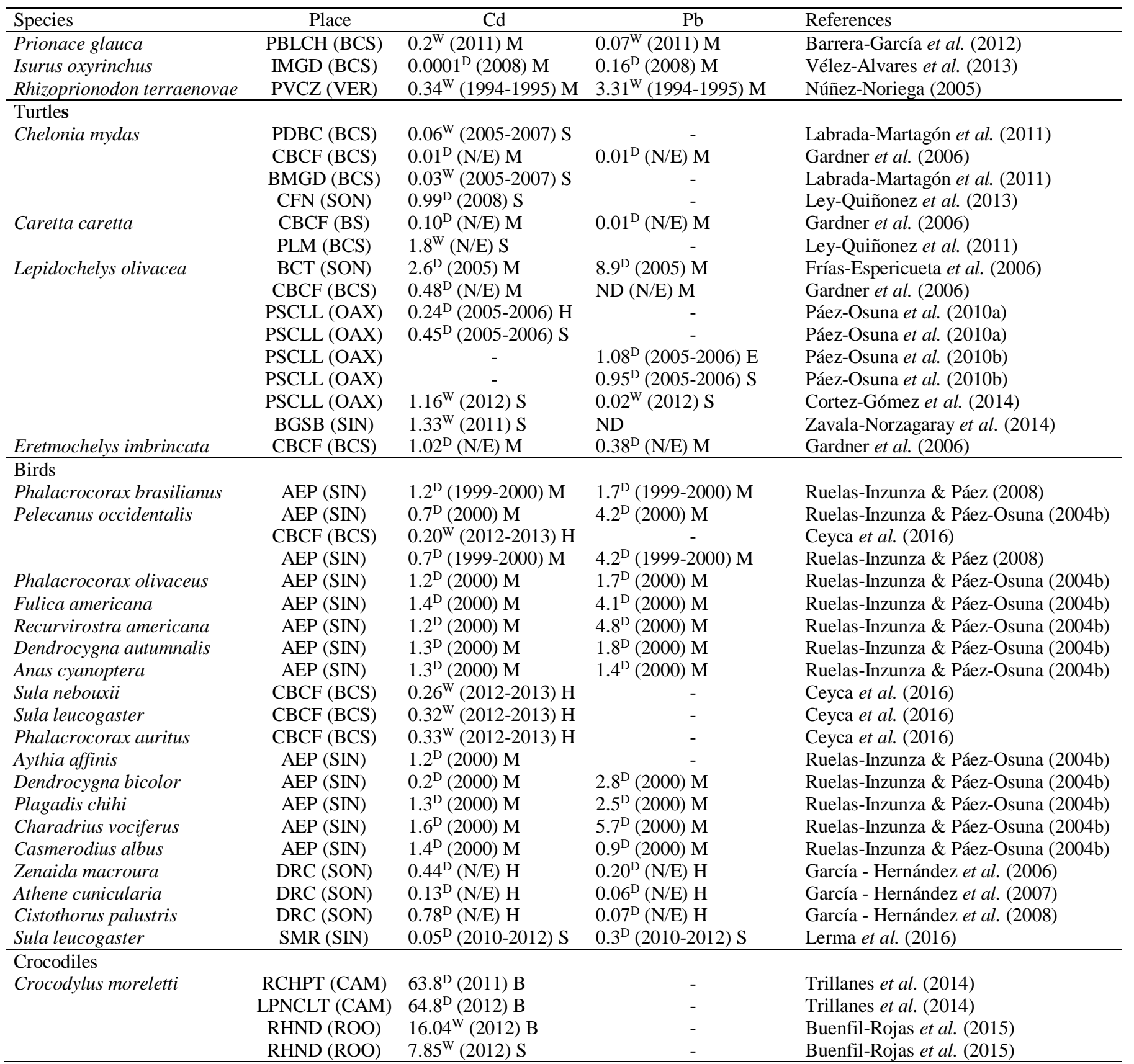

tration was found $\left(<0.001 \mu \mathrm{g} \mathrm{g}^{-1} \mathrm{dw} ; 2003\right)$, while the highest concentration was found in El Tobari lagoon (0.47 $\left.\mu \mathrm{g} \mathrm{g}^{-1} \mathrm{dw} ; 2009\right)$. Studies conducted on Mytilopsis sallei in Chetumal Bay, in Quintana Roo, reported values of $1.09 \mu \mathrm{g} \mathrm{g}^{-1} \mathrm{dw}$ (2002), the highest reported values in Mexico during the review period. The only studies on Hg's concentration in bivalves of inland ecosystems were made with the genus Corbicula. One study was conducted in the Colorado River, in Sonora, and another in the Coatzacoalcos
River, in Veracruz. The values reported were $<0.04$ and $0.09 \mu \mathrm{g} \mathrm{g}^{-1} \mathrm{dw}$, respectively. Hg's concentration values reported in Mexico's bivalves are similar to those reported by other authors in other parts of the world. For example, Astudillo et al. (2005), in Ruelas-Inzunza et al. (2013a), reported $\mathrm{Hg}$ values of $0.04 \mu \mathrm{g} \mathrm{g}^{-1} \mathrm{dw}$ in Crassostrea sp. collected in the Gulf of Paria, Venezuela. Vaisman et al. (2005), in Ruelas-Inzunza et al. (2014), reported values of 0.04, 0.05, 0.08, and 0.15 $\mu \mathrm{g} \mathrm{g}^{-1} \mathrm{dw}$ in Crassostrea rhizophorae collected in the 
Pacoti estuary, the Coco estuary, and Ceara estuary in Brazil, respectively. Zorita et al. (2007), in RuelasInzunza et al. (2013a) reported values of $0.23 \mu \mathrm{g} \mathrm{g}^{-1} \mathrm{dw}$ in Mytilus galloprovincialis collected in the northeast of the Mediterranean Sea. Concerning inland ecosystems, Neufeld (2010), and Faria et al. (2010), in RuelasInzunza et al. (2014), reported $\mathrm{Hg}$ values of 1.89 and $2.3 \mu \mathrm{g} \mathrm{g}^{-1} \mathrm{dw}$ in Corbicula fluminea collected in the Norte river in the USA and the Ebro river in Spain, respectively.

The content of $\mathrm{Hg}$ in crustaceans was evaluated mainly in marine organisms. Concerning inland ecosystems, the only cases found were those of Procambarus clarkii and Palaemonetes paludosus, collected in the state of Sonora, in the Colorado River, the Bocana Lagoon, and the Santa Clara swamp, with values of $0.40,0.05$, and $0.69 \mu \mathrm{g} \mathrm{g}^{-1} \mathrm{dw}$ in the whole organism, respectively. These values are similar to others reported worldwide; for example, Li et al. (2015) reported $\mathrm{Hg}$ concentrations in Macrobrachium nipponense of $0.03,0.04$, and $0.03 \mu \mathrm{g} \mathrm{g}^{-1} \mathrm{dw}$ in three reservoirs located in the central part of China. These values, however, are lower than concentrations found in other freshwater crustaceans collected in contaminated areas. For example, Higueras et al. (2006) reported values of $\mathrm{Hg}>9.06 \mu \mathrm{g} \mathrm{g}^{-1} \mathrm{dw}$ in the muscle of $P$. clarkii collected in an area impacted by mining activities in central Spain (Almaden), and Suárez-Serrano et al. (2010) reported $\mathrm{Hg}$ concentrations of $1.5 \mu \mathrm{g} \mathrm{g}^{-1} \mathrm{dw}$ in $P$. clarkii collected in an area impacted by industrial waste in the Ebro river, Spain. Concerning marine crustaceans, DelgadoÁlvarez et al. (2015a) monitored Hg's concentration in muscle tissue of Penaeus vannamei grown in aquaculture farms in the states of Sonora, Sinaloa, and Nayarit. They found concentration levels of $0.19,0.31$, and $0.45 \mu \mathrm{g} \mathrm{g}^{-1} \mathrm{dw}$ respectively, similar to those found in $P$. vannamei by Ruelas-Inzunza et al. (2004) $(0.2 \mu \mathrm{g}$ $\left.\mathrm{g}^{-1} \mathrm{dw}\right)$ and Ruelas-Inzunza et al. (2011a) $\left(0.42 \mu \mathrm{g} \mathrm{g}^{-1}\right.$ $\mathrm{dw}$ ) in the Altata-Ensenada del Pabellón Lagoon complex in the state of Sinaloa. Similar concentrations have been reported in other species of penaeids in this lagoon complex (Table 1), which suggests that these organisms have similar responses to $\mathrm{Hg}$ exposure. García-Hernández et al. (2015) conducted a monitoring study of Hg's concentration in crustaceans from thirteen coastal ecosystems in Sonora. Sinaloa's states focused on the Callinectes bellicosus crab, in which they found concentrations ranging from 0.07 up to $1.8 \mu \mathrm{g} \mathrm{g}^{-1} \mathrm{dw}$ in muscle tissue. The highest concentrations were found in crabs from Santo Tomas Bay, with $1.82 \mu \mathrm{g} \mathrm{g}^{-1} \mathrm{dw}$. The mercury concentration in these ecosystems' sediments, determined in the same study, was $<0.001$ $\mu \mathrm{g} \mathrm{g}^{-1}$, while the concentration in water (total fraction) was $5.2 \mu \mathrm{g} \mathrm{L}^{-1}$. The authors indicate the importance of the water matrix in the content of $\mathrm{Hg}$ in C. bellicosus; however, the highest concentrations of $\mathrm{Hg}$ in the water were found in the Guaymas-Empalme Bay, with $6.3 \mu \mathrm{g}$ $\mathrm{g}^{-1}$, while the $\mathrm{Hg}$ concentrations in C. bellicosus were the lowest, with $0.56 \mu \mathrm{g} \mathrm{g}^{-1} \mathrm{dw}$. On the other hand, the highest $\mathrm{Hg}$ concentration in sediment was found in Las Guásimas Bay, with $1.23 \mu \mathrm{g} \mathrm{g}^{-1} \mathrm{dw}$, while the concentration in C. bellicosus was $0.63 \mu \mathrm{g} \mathrm{g}^{-1} \mathrm{dw}$ showing the complex interaction between organisms and environmental pollutants.

Teleost fish are the most heterogeneous group of organisms used as biomonitors of aquatic pollution in Mexico. Marine and freshwater fish from different ecosystems (rivers, lakes, lagoons, estuaries, bays) and different eating habits (planktivorous, detritivorous, omnivorous, and carnivorous) have been used for this purpose. The present review shows that $\mathrm{Hg}$ concentration in fish may vary depending on the type of ecosystem, geographical location (anthropogenic or geological influence), eating habits (trophic level), and species. In the case of rivers, the highest concentration of $\mathrm{Hg}$ was found in muscle tissue of Lepomis macrochirus, in the Hardy river, with $3.2 \mu \mathrm{g} \mathrm{g}^{-1} \mathrm{dw}$, followed by Gobomorius polylepis in the Coatzacoalcos river, with $0.86 \mu \mathrm{g} \mathrm{g}^{-1} \mathrm{dw}$, then Pterygoplichthys pardalis in the Usumacinta River, with $0.45 \mu \mathrm{g} \mathrm{g}^{-1} \mathrm{dw}$, and Mugil sp., in the Santiago River, with $0.20 \mu \mathrm{g} \mathrm{g}^{-1} \mathrm{dw}$. Concerning lakes and other reservoirs, the highest concentrations of $\mathrm{Hg}$ were found in Lake Chapala in muscle tissue of Cyprinus sp., with $4.15 \mu \mathrm{g} \mathrm{g}^{-1} \mathrm{dw}$ in $2007,1.95 \mu \mathrm{g} \mathrm{g}^{-1} \mathrm{dw}$ in $2010,1.75 \mu \mathrm{g}$ $\mathrm{g}^{-1} \mathrm{dw}$ in 2011, and $0.5 \mu \mathrm{g} \mathrm{g}^{-1} \mathrm{dw}$ in 2012. In other sites, such as the Luis León Dam, the concentration was 0.04 $\mu \mathrm{g} \mathrm{g}^{-1} \mathrm{dw}$ in 2011-2012, and not detected (ND) in the Viejo Mandin Dam in 2013.

Coastal ecosystems have a greater diversity of fish communities, harboring different trophic levels through which energy (and pollutants) is transferred within the same community. It is possible to observe an increase in the $\mathrm{Hg}$ levels in muscle from the lower trophic levels to higher levels. In the Urias Lagoon, for example, the concentration of $\mathrm{Hg}$ in muscle tissue increased from $0.1-0.18 \mu \mathrm{g} \mathrm{g}^{-1} \mathrm{dw}$ in Mugil sp. to $0.7 \mu \mathrm{g} \mathrm{g}^{-1} \mathrm{dw}$ in Eugerris axillaris and $0.9 \mu \mathrm{g} \mathrm{g}^{-1} \mathrm{dw}$ in Lutjanus colorado; the latter two have a predator-prey relationship with Mugil sp., at least in their earlier stages (Jara-Marini et al., 2012). The same trend can be observed in other places, such as Altata-Ensenada del Pabellón, where Hg's concentration went from 0.13$0.20 \mu \mathrm{g} \mathrm{g}^{-1} \mathrm{dw}$ in muscle tissue of $M$. cephalus to 0.89 $\mu \mathrm{g} \mathrm{g}^{-1} \mathrm{dw}$ in the muscle of L. colorado. In Santa Maria$\mathrm{La}$ Reforma, the $\mathrm{Hg}$ concentration went from $0.13 \mu \mathrm{g} \mathrm{g}^{-1}$ $\mathrm{dw}$ in muscle tissue of $M$. curema to $0.82 \mu \mathrm{g} \mathrm{g}^{-1} \mathrm{dw}$ in 
C. medius's muscle. In Topolobampo, the concentration $\mathrm{Hg}$ in muscle tissue of M. cephalus was $0.03 \mu \mathrm{g} \mathrm{g}^{-1} \mathrm{dw}$, increasing to $3.32 \mu \mathrm{g} \mathrm{g}^{-1} \mathrm{dw}$ in the muscle of $C$. caninus. Other cases in which the level of mercury increased from lower to higher trophic levels were found in Guaymas (Sonora), Barra de Navidad Lagoon (Jalisco), and the estuary of the Coatzacoalcos River (Veracruz) (Table 1). There were also cases where the trend mentioned above was not clearly seen. In the Alvarado lagoon, for example, in Veracruz's state, the concentration of $\mathrm{Hg}$ in muscle tissue of $M$. curema was $2.85 \mathrm{\mu g} \mathrm{g}^{-1} \mathrm{dw}$ (2003), while the concentration of $\mathrm{Hg}$ in C. parallelus was $0.75-0.9 \mu \mathrm{g} \mathrm{g}^{-1} \mathrm{dw}$ (2000-2003). In general, top predators such as the great amberjack (Seriola lalandi), the striped marlin (Tetrapturus audax), the wahoo (Acanthocybium solandri), the blue marlin (Makaira mazara), and the Indo-Pacific sailfish (Istiophorus platypterus) had higher concentrations of mercury.

In coastal ecosystems, elasmobranchs are considered top predators, making them susceptible to pollutants' biomagnification such as mercury (RuelasInzunza et al., 2013b). Most of the studies analyzed in this review focused on rays and sharks were conducted in the Gulf of California region. Among sharks, Sphyrna zygaena showed the highest average $\mathrm{Hg}$ concentration, with $33 \mu \mathrm{g} \mathrm{g}^{-1} \mathrm{dw}$ in muscle for 2003 2004; however, other studies reported concentrations of $0.98 \mu \mathrm{g} \mathrm{g}^{-1} \mathrm{dw}$ of $\mathrm{Hg}$ in muscle tissue of the same species in 2001-2005 (Table 1). Other species such as S. lewini, Prionace glauca, C. leucas, C. falciformis, and Rhizoprionodon longurio showed average concentrations of $0.49-4.32,1.96-5.56,0.2-2.48,1.2-$ 3.4 , and 3.6-5.2 $\mu \mathrm{g} \mathrm{g}^{-1} \mathrm{dw}$ of $\mathrm{Hg}$ in muscle tissue, respectively. Regarding the other regions of Mexico, only one study was found for the Gulf of Mexico region; Núñez-Noriega (2005) analyzed the concentrations of $\mathrm{Hg}$ in muscle tissue of C. limbatus sampled in the port of Veracruz, finding average concentrations of $13.32 \mu \mathrm{g} \mathrm{g}^{-1} \mathrm{dw}$, higher than those found in C. limbatus from the Gulf of California (2.04 $\mu \mathrm{g} \mathrm{g}{ }^{-1} \mathrm{dw}$ in muscle). Núñez-Noriega (2005) also analyzed the concentration of mercury in $R$. terraenovae, obtaining average values of $3.04 \mu \mathrm{g} \mathrm{g}^{-1}$ $\mathrm{dw}$, which were lower than those found in similar species from the Gulf of California, such as $R$. longurio (5.2 $\mu \mathrm{g} \mathrm{g}^{-1} \mathrm{dw}$ of $\mathrm{Hg}$ in muscle).

The average concentration of mercury in the muscle tissue of the batoids (rays and torpedoes) was lower than the concentration found in sharks (approximately 1.23 and $4.01 \mu \mathrm{g} \mathrm{g}^{-1} \mathrm{dw}$ of $\mathrm{Hg}$, respectively), probably because sharks belong to a level highest trophic. Sharks feed mainly on fish, and batoids feed on organisms with lower trophic levels, such as crustaceans and mollusks and, to a lesser extent, on fish (Ruelas-Inzunza et al., 2013b), which could explain the differences in $\mathrm{Hg}$ levels. The highest concentrations were found in Dasyatis longa, with $2.84 \mu \mathrm{g} \mathrm{g}^{-1} \mathrm{dw}$, in 2003-2004, and $4.46 \mu \mathrm{g} \mathrm{g}^{-1} \mathrm{dw}$ in 2012. Urolophus spp. batoid's muscle had concentrations of $\mathrm{Hg}$ in the muscle of 1.37-3.71 $\mu \mathrm{g}$ $\mathrm{g}^{-1} \mathrm{dw}$ in 2012. The lowest concentrations were found in Mobula japanica in 2012, with 0.04-0.56 $\mu \mathrm{g} \mathrm{g}^{-1} \mathrm{dw}$.

Other groups of aquatic animals that have also been used as biomonitors of aquatic pollution in Mexico include sea turtles, aquatic birds, crocodiles, and marine mammals. Table 1 shows the average mercury concentrations reported in sea turtles, aquatic birds, crocodiles, and marine mammals. Regarding aquatic birds, piscivorous species such as Pelecanus occidentalis and Phalacrocorax brasilianus had higher concentrations than non-piscivorous birds such as Anas discors and A. clypeata (Table 1). In Mexico, studies with aquatic reptiles such as sea turtles and crocodiles are very scarce. Only two studies with crocodiles were found, by Trillanes et al. (2014) and Buenfil-Rojas et al. (2015), who reported mercury concentrations of 3.1$4.3 \mu \mathrm{g} \mathrm{g} \mathrm{g}^{-1} \mathrm{dw}$ in Crocodylus moreletti in the Champotón-Petenes-Celestún River, and of $0.33 \mu \mathrm{g} \mathrm{g}^{-1}$ ww in the Hondo River, respectively. Only three studies with sea turtles were found, one for the Gulf of California area (Chelonia mydas, C. caretta, and Lepidochelys olivacea), another for the Central Pacific area (L. olivacea), and another for the Gulf of Mexico area (C. mydas and Eretmochelys imbricata). Different tissues (muscle, egg, and blood) were used in these studies to evaluate the concentration of pollutants, which could serve as the basis for future research.

\section{Cadmium and lead in biota}

Table 2 shows the concentrations of $\mathrm{Cd}$ and $\mathrm{Pb}$ in aquatic organisms. As in the case of macrophytes, most of the studies about the presence of $\mathrm{Cd}$ and $\mathrm{Pb}$ in biota focused on the Gulf of California area, but a higher number of biomonitoring studies focused on $\mathrm{Cd}$ and $\mathrm{Pb}$. Several sites along the Gulf of California coast were monitored using macrophytes, finding average concentrations of Cd of 0.14-6.2 $\mu \mathrm{g} \mathrm{g}^{-1} \mathrm{dw}$, and 0.19-8.8 $\mu \mathrm{g} \mathrm{g}^{-1}$ $\mathrm{dw}$ of $\mathrm{Pb}$. The highest average concentrations of $\mathrm{Cd}$ were found in Rupia maritima $\left(6.2 \mu \mathrm{g} \mathrm{g}^{-1} \mathrm{dw}\right)$ collected in the San Ignacio Lagoon (Baja California Sur), while the lowest concentrations were found in Caulerpa sertularioides $\left(0.14 \mu \mathrm{g} \mathrm{g}^{-1} \mathrm{dw}\right)$ collected in the Urías Lagoon (Sinaloa). In Pb's case, the lowest average concentrations were found in Gracilaria subsecundata $\left(0.19 \mu \mathrm{g} \mathrm{g}^{-1} \mathrm{dw}\right)$ in Altata-Ensenada del Pabellón, while the highest concentrations were found in $R$. maritima $\left(8.8 \mu \mathrm{g} \mathrm{g}^{-1} \mathrm{dw}\right)$ collected in the San Ignacio Lagoon. There were also cases, such as those reported by 
Orduña-Rojas \& Longoria-Espinoza (2006), in which average $\mathrm{Pb}$ concentrations of $174 \mu \mathrm{g} \mathrm{g}^{-1} \mathrm{dw}$ was found in $U$. lactuca collected in the Ignacio-Navachiste Lagoon (Sinaloa), suggesting the possible influence of the small fishing boats used in the region. Of the sites evaluated in the coastal area of the Gulf of California, the ones with the lowest concentrations of $\mathrm{Cd}$ and $\mathrm{Pb}$, simultaneously, were the Guaymas bay with 0.54 and $0.35 \mu \mathrm{g} \mathrm{g}^{-1} \mathrm{dw}$, and Altata-Ensenada del Pabellón with 0.18 and $0.19 \mu \mathrm{g} \mathrm{g}^{-1} \mathrm{dw}$ in G. subsecundata and $U$. lactuca, respectively, both species collected in the period 1998-1999. In general, the concentration of $\mathrm{Cd}$ and $\mathrm{Pb}$ varied from one species to another. For example, Riosmena-Rodríguez et al. (2010) reported concentrations of $3.16,1.36$, and $4.5 \mu \mathrm{g} \mathrm{g}^{-1} \mathrm{dw}$ for $\mathrm{Cd}$ and $0.9,0.8$, and $2.15 \mu \mathrm{g} \mathrm{g}^{-1} \mathrm{dw}$ for $\mathrm{Pb}$ in G. textorii, $G$. vermiculophylla, and $R$. maritima, respectively, collected in the Banderitas Estuary (Baja California Sur) in 2004-2005. Ruelas-Inzunza \& Páez-Osuna (2006) reported concentrations of $0.18,0.23$, and 0.87 $\mu \mathrm{g} \mathrm{g}^{-1} \mathrm{dw}$ for $\mathrm{Cd}$ and $0.19,4.9$, and $3.1 \mu \mathrm{g} \mathrm{g}^{-1} \mathrm{dw}$ for $\mathrm{Pb}$ in G. subsecundata, Gracilaria sp., and Polysiphonia sp., respectively, collected in Altata-Ensenada del Pabellón (Sinaloa) in 2004-2005. There were only a few other studies that used macrophytes in other areas of the country. Avelar et al. (2013) reported $\mathrm{Cd}$ concentrations of $0.72 \mu \mathrm{g} \mathrm{g}^{-1} \mathrm{dw}$ in $T$. testudinum in the Yalahau Lagoon, an area with relatively little contamination in the Mexican Caribbean; however, the authors reported maximum concentrations of $\mathrm{Cd}$ higher than $5 \mu \mathrm{g} \mathrm{g}^{-1} \mathrm{dw}$ in leaves of Thalassia testudinum, suggesting how important underground infiltration of contaminants is in this type of sites. It is thought that macrophytes reflect greater accuracy in the amount of contamination in the fraction dissolved in the water column (Orduña-Rojas \& Longoria-Espinoza, 2006). Natural sources such as upwellings and hydrothermal chimneys (Riosmena-Rodríguez et al., 2010), or anthropic sources such as gasoline in fishing vessels (Soto-Jiménez et al., 2008), can significantly alter the content of metals in macrophytes.

Studies of the content of $\mathrm{Cd}$ and $\mathrm{Pb}$ in bivalves were the most abundant. Close to 30 sites on the Gulf of California's coastal area were studied using bivalves as biomonitors. The average concentrations of $\mathrm{Cd}$ and $\mathrm{Pb}$ were $0.22-17.23$ and $0.001-17.4 \mu \mathrm{g} \mathrm{g}^{-1} \mathrm{dw}$, respectively; countrywide, the highest concentrations of both $\mathrm{Cd}$ and $\mathrm{Pb}$ were found in this area. The typically high $\mathrm{Cd}$ concentrations present in the Gulf of California area sites were mainly associated with upwelling events that occurred in the winter season. Phosphorus pesticides and fertilizers, used for more than 50 years in intensive agriculture in this area, have generated large quantities of residues rich in $\mathrm{Cd}$ and other metals, which have been deposited in the continental slope's sediments, and reintroduced to the coastal zone during upwelling events. The sites with the highest average $\mathrm{Cd}$ concentrations were Bacochibampo Bay and Kuu Kaak Bay, 22.54, and $57.12 \mu \mathrm{g} \mathrm{g}^{-1} \mathrm{dw}$, respectively; these two sites are frequently affected by upwelling events. As we have seen before, the ability to accumulate pollutants varies from one species to another; for example, García-Hernández et al. (2005) found $\mathrm{Cd}$ levels of $0.4,0.4,2.44$, and $8.16 \mu \mathrm{g} \mathrm{g}^{-1} \mathrm{dw}$ in Megapitaria squalida, Chione californiensis, Pinna rugosa and Anadara multicostata collected in the Kuu Kaak Bay (Sonora), respectively. The highest average concentrations of $\mathrm{Pb}$ in bivalves living on the coastal area of the Gulf of California were found mostly in sites with intense fishing activity, mainly in the Sinaloa coast, where the highest concentrations were associated with the fuel used by small fishing boats. Other studies that used bivalves were conducted in areas outside the Gulf of California. For example, Segovia-Zavala et al. (2004) reported concentrations of $17.23 \mu \mathrm{g} \mathrm{g}^{-1} \mathrm{dw}$ of $\mathrm{Cd}$ in Mytilus californianus transplanted to Punta Banda (Baja California Norte), an area affected by upwelling events. Castañeda-Chavez et al. (2014) reported concentrations of 42 and $189 \mu \mathrm{g} \mathrm{g}^{-1} \mathrm{dw}$ of $\mathrm{Cd}$ and $\mathrm{Pb}$, respectively, in $C$. virginica collected in the Mecoacán Lagoon in the state of Veracruz, in an area historically impacted by the Petrochemical industry. Nearly $80 \%$ of the cases showed average concentrations of 0.2-6.9 $\mu \mathrm{g} \mathrm{g}^{-1} \mathrm{dw}$ for $\mathrm{Cd}$ and 0.001-6.8 $\mu \mathrm{g} \mathrm{g}^{-1}$ $\mathrm{dw}$ for $\mathrm{Pb}$, similar to values reported worldwide. For example, Lino et al. (2016) reported Cd concentrations of 0.15 and $0.6 \mu \mathrm{g} \mathrm{g}^{-1} \mathrm{dw}$ in Perna perna from Sepitaba Bay and Cabo do Arraial, and 1.2 and $0.8 \mu \mathrm{g} \mathrm{g}^{-1} \mathrm{dw}$ of $\mathrm{Cd}$ in Nodipecten nodosus from Ilha Grande Bay and Cabo do Arraial, in southern Brazil. Liu et al. (2017) reported average concentrations of 5.9-7.8 $\mu \mathrm{g} \mathrm{g}^{-1} \mathrm{dw}$ for $\mathrm{Cd}$ and $0.4-0.5 \mu \mathrm{g} \mathrm{g}^{-1} \mathrm{dw}$ for $\mathrm{Pb}$ in muscle tissue of Mactra veneriformis in the Laizhou Bay in China. It is worth remembering that China and Brazil are countries with emerging economies and intense industrial activity. About $10 \%$ of the cases showed high concentrations of $\mathrm{Pb}$ and $\mathrm{Cd}: 10-34 \mu \mathrm{g} \mathrm{g}^{-1} \mathrm{dw}$ and 10-57 $\mu \mathrm{g} \mathrm{g}^{-1} \mathrm{dw}$ for $\mathrm{Cd}$, respectively. These atypically high concentrations were influenced by upwelling events, fishing activities, or contaminated urban or industrial effluents.

Average $\mathrm{Cd}$ and $\mathrm{Pb}$ levels in crustaceans are shown in Table 2. There were very few studies on the concentration of $\mathrm{Cd}$ and $\mathrm{Pb}$ in freshwater crustaceans. Mendoza-Carranza et al. (2016) evaluated Cd's concentration in muscle tissue of Macrobrachium acanthurus, finding values of $0.72 \mu \mathrm{g} \mathrm{g}^{-1} \mathrm{dw}$ in the San Pedrito Lagoon, Tabasco, in the year 2009. Similarly, 
García-Hernández et al. (2001) found average wholebody concentrations of 0.2 and $0.4 \mu \mathrm{g} \mathrm{g}^{-1} \mathrm{dw}$ for $\mathrm{Cd}$ and 0.001 and $1.8 \mu \mathrm{g} \mathrm{g}^{-1} \mathrm{dw}$ for $\mathrm{Pb}$ in Procambarus clarkii and Palaemonetes paludosus. These values were lower than other values reported in other areas of the world for freshwater crustaceans. For example, Rabiul-Islam et al. (2017) reported values of 2.38 and $4.64 \mu \mathrm{g} \mathrm{g}^{-1} \mathrm{dw}$ of $\mathrm{Cd}$ and $\mathrm{Pb}$, respectively, in muscle tissue of $M$. rosenbergii collected in the Satkhira Basin in southern Bangladesh. In coastal ecosystems, the only studies found in this review focused on the Gulf of California and Mexico's Gulf. Lango-Reynoso et al. (2013) found concentrations of 0.05 and $0.4 \mu \mathrm{g} \mathrm{g}^{-1} \mathrm{dw}$ for $\mathrm{Cd}$ and 0.5 and $0.5 \mu \mathrm{g} \mathrm{g}^{-1} \mathrm{dw}$ for $\mathrm{Pb}$ in muscle tissue of Penaeus setiferus and Farfantepenaeus aztecus, respectively, in the Alvarado Lagoon (Veracruz). Vázquez et al., 2001 found concentrations of $\mathrm{Cd}$ and $\mathrm{Pb}$ of 3.64 and $4.22 \mu \mathrm{g}$ $\mathrm{g}^{-1} \mathrm{dw}$, respectively, in muscle tissue of $F$. aztecus collected on the coast of Campeche. Aguilar-Ucán et al. (2014) reported concentrations of $\mathrm{Cd}$ and $\mathrm{Pb}$ of 0.25 and $1.7 \mu \mathrm{g} \mathrm{g}^{-1} \mathrm{dw}$, respectively, in muscle tissue of Callinectes sapidus collected in the Terminos Lagoon (Campeche). Soto-Jiménez et al. (2008) reported concentrations of $\mathrm{Pb}$ of $23 \mu \mathrm{g} \mathrm{g}^{-1} \mathrm{dw}$ in muscle tissue of $C$. arcuatus collected in the Urías Lagoon (Sinaloa). No other studies were found for other blue crab species; however, several studies were conducted throughout Sinaloa and Nayarit using shrimp as biomonitors, with average muscle concentrations of 0.36-4.94 $\mu \mathrm{g} \mathrm{g}^{-1} \mathrm{dw}$ for $\mathrm{Cd}$ and 0.4-6.39 $\mu \mathrm{g} \mathrm{g}^{-1} \mathrm{dw}$ for $\mathrm{Pb}$. In general, the concentration of $\mathrm{Cd}$ and $\mathrm{Pb}$ varied between different tissues. For example, Frías-Espericueta et al. (2009) reported values of $0.36-0.76 \mu \mathrm{g} \mathrm{g}^{-1} \mathrm{dw}$ for $\mathrm{Cd}$ and 4.21 $6.93 \mu \mathrm{g} \mathrm{g}^{-1} \mathrm{dw}$ for $\mathrm{Pb}$ in muscle tissue of $P$. vannamei collected in six lagoons in the coastal zone of Sinaloa and Nayarit, while in hepatopancreas, they reported values of 1.10-7.97 $\mu \mathrm{g} \mathrm{g}^{-1} \mathrm{dw}$ for $\mathrm{Cd}$ and 5.45-18.84 $\mu \mathrm{g}$ $\mathrm{g}^{-1} \mathrm{dw}$ for Pb. Similarly, Vázquez et al. (2001) reported values of 3.6 and $4.2 \mu \mathrm{g} \mathrm{g}^{-1} \mathrm{dw}$ for $\mathrm{Pb}$ and $\mathrm{Cd}$ respectively in muscle tissue of $P$. setiferus, and 15.03 and $11.6 \mu \mathrm{g} \mathrm{g}^{-1} \mathrm{dw}$ for $\mathrm{Cd}$ and $\mathrm{Pb}$ respectively in hepatopancreas, results that indicate the high capacity of this organ to bioaccumulate metals.

Table 2 shows the concentrations of $\mathrm{Cd}$ and $\mathrm{Pb}$ in fish. In general, the number of $\mathrm{Cd}$ and $\mathrm{Pb}$ biomonitoring studies in fish was considerably lower than $\mathrm{Hg}^{\prime} \mathrm{s}$ biomonitoring studies. In the case of freshwater fish, the average concentrations of $\mathrm{Cd}$ in muscle tissue were $0.28-2.24 \mu \mathrm{g} \mathrm{g}^{-1} \mathrm{dw}$; for $\mathrm{Pb}$, the average concentrations were $<0.001-0.55 \mu \mathrm{g} \mathrm{g}^{-1} \mathrm{dw}$ in ecosystems located in southeastern Mexico. The highest $\mathrm{Pb}$ concentrations were $9.6 \mu \mathrm{g} \mathrm{g}^{-1} \mathrm{dw}$ in muscle tissue of Dormitator latifrons collected in the Tres Palos Lagoon, in state of Veracruz. Average levels of $\mathrm{Cd}$ and $\mathrm{Pb}$ of 0.28 and 2.12 $\mu \mathrm{g} \mathrm{g}^{-1} \mathrm{dw}$, respectively, were also found in the muscle tissue of Oreochromis aureus collected in the Salto Dam (Sinaloa). $\mathrm{Cd}$ and $\mathrm{Pb}$ concentrations in marine fish's muscle tissue belonging to the lower trophic levels were lower than those found in crustaceans collected in the same areas. For example, FríasEspiricueta et al. (2011) evaluated Cd and Pb's concentration in muscle tissue of Mugil cephalus collected in seven coastal lagoons located in the state of Sinaloa and found values of $0.13-0.26$ and $1.38-2.10 \mu \mathrm{g}$ $\mathrm{g}^{-1} \mathrm{dw}$, respectively. In the same sites, Frías-Espiricueta et al. (2009) reported levels of 0.36-0.76 and 4.21-6.93 $\mu \mathrm{g} \mathrm{g}^{-1} \mathrm{dw}$ for $\mathrm{Cd}$ and $\mathrm{Pb}$, respectively, in muscle tissue of $P$. vannamei. Concerning predatory fish, Vázquez et al. (2001) reported values of $<0.001$ and $1.6 \mu \mathrm{g} \mathrm{g}^{-1} \mathrm{dw}$ for $\mathrm{Cd}$ and $\mathrm{Pb}$, respectively, in muscle tissue of Syacium gunteri. In muscle tissue of $P$. zetiferus, the same authors reported values of 3.6 and $4.2 \mu \mathrm{g} \mathrm{g}^{-1} \mathrm{dw}$; however, in the case of Lutjanus analis, the reported concentrations were $<0.001$ for $\mathrm{Cd}$ and 4.68 for $\mathrm{Pb} \mu \mathrm{g}$ $\mathrm{g}^{-1} \mathrm{dw}$. Similarly, Ruelas-Inzunza \& Páez-Osuna (2008) reported values of 3.1 and $0.5 \mu \mathrm{g} \mathrm{g}^{-1} \mathrm{dw}$ for $\mathrm{Cd}$ and $\mathrm{Pb}$ in muscle tissue of $P$. vannamei, respectively, and 0.5 and $0.9 \mu \mathrm{g} \mathrm{g}^{-1} \mathrm{dw}$ in P. stylirostris. In $L$. colorado and Cynoscion xanthulus, the reported $\mathrm{Cd}$ levels were 0.2 and $0.9 \mu \mathrm{g} \mathrm{g}^{-1} \mathrm{dw}$, while the values of and $\mathrm{Pb}$ were 1.3 and $2.6 \mu \mathrm{g} \mathrm{g}^{-1} \mathrm{dw}$, respectively. These results are consistent with the low transfer efficiency from lower to higher trophic levels, and even with the predator-prey ratio, at least in the case of fish.

The number of biomonitoring studies focused on $\mathrm{Cd}$ and $\mathrm{Pb}$ 's concentration in elasmobranchs was considerably lower than the number of studies focused on mercury and other biomonitors. In the Gulf of California, the reported levels of $\mathrm{Cd}$ and $\mathrm{Pb}$ were $<0.001-1.48 \mu \mathrm{g} \mathrm{g}^{-1} \mathrm{dw}$ for $\mathrm{Cd}$ and 0.16-0.28 $\mu \mathrm{g} \mathrm{g}^{-1} \mathrm{dw}$ for $\mathrm{Pb}$. In the Gulf of Mexico, the reported levels were 1.4-1.48 $\mu \mathrm{g} \mathrm{g}^{-1} \mathrm{dw}$ for $\mathrm{Cd}$ and 10.04-13.24 $\mu \mathrm{g} \mathrm{g}^{-1} \mathrm{dw}$ for $\mathrm{Pb}$; it is worth noting that the latter value of $\mathrm{Pb}$ was approximately 40 times higher than the value found in the Gulf of California. As in other fish, the concentrations of $\mathrm{Cd}$ and $\mathrm{Pb}$ in the muscle tissue of sharks were not higher than the concentrations found in organisms of lower trophic levels. The tendency to bioaccumulate higher $\mathrm{Cd}$ and $\mathrm{Pb}$ concentrations in the liver than in muscle was also observed in sharks. Barrera-Garcia et al. (2013), for example, reported concentrations of $\mathrm{Cd}$ in the liver nearly 140 times higher than the concentrations reported in muscle by Barrera-Garcia et al. (2012). Terrazas-López et al. (2016) also reported $\mathrm{Cd}>900$ times higher in the liver, probably due to the high content of lipids in the liver and to its ability to synthesize metallothioneins (rich in cysteine), which are present in lower quantities in the 
muscles. In general, $\mathrm{Cd}$ and $\mathrm{Pb}$ tended to bioconcentrate in the liver and to biodilute in muscle.

The concentration of $\mathrm{Cd}$ and $\mathrm{Pb}$ in sea turtles, waterfowl, and crocodiles is shown in Table 2. The average concentration of $\mathrm{Cd}$ in muscle tissue of sea turtles sampled in the Gulf of California ranged from 0.01 to $2.6 \mu \mathrm{g} \mathrm{g}^{-1} \mathrm{dw}$, while the average concentration of $\mathrm{Pb}$ ranged from not detect to $8.9 \mu \mathrm{g} \mathrm{g}^{-1} \mathrm{dw}$. In blood, $\mathrm{Cd}$ levels were ranged between 0.15 and $6.65 \mu \mathrm{g} \mathrm{g}^{-1} \mathrm{dw}$. In the central area of the Mexican Pacific, Cd levels ranged from 0.45 to $0.85 \mu \mathrm{g} \mathrm{g}^{-1} \mathrm{dw}$. The transfer of contaminants between mother and offspring is a current subject of study, but relatively little is known about the effect that mother-transmitted contaminants have on the offspring's development. Páez-Osuna et al. (2010a) found in Lepidochelys olivacea, that the maternal transfer of Cd was $0.20 \%$ of the Cd content in the whole body (for a spawn of 200 eggs). García-Hernández et al. (2006) evaluated the concentration of $\mathrm{Cd}$ and $\mathrm{Pb}$ in eggs and embryos (in different stages) of aquatic birds living in the Colorado River Delta (Sonora) and found concentrations of $0.13-0.78$ and $0.06-0.20 \mu \mathrm{g} \mathrm{g}^{-1} \mathrm{dw}$ for $\mathrm{Cd}$ and $\mathrm{Pb}$ respectively. Similarly, Ceyca et al. (2016) reported values of 1-1.6 $\mu \mathrm{g} \mathrm{g}^{-1} \mathrm{dw}$ for $\mathrm{Cd}$ in seabird eggs in the coastal zone of Sinaloa, similar values to those found in the muscle tissue of several seabirds collected in the Altata-Ensenada de Pabellón Lagoon (Sinaloa), with concentrations of $0.2-1.6 \mu \mathrm{g} \mathrm{g}^{-1} \mathrm{dw}$, which suggests that a high percentage of this contaminant is transferred via the mother.

\section{Organochlorine pesticides (POCs) in aquatic organisms}

The results of the average concentration of POCs in different aquatic organisms in Mexico are shown (Table 3). The number of biomonitoring studies of POCs was considerably lower than the number of heavy metals' biomonitoring studies. The most studied compound was $\sum$ DDTs. In the case of marine mollusks, the highest concentrations ( $282 \mathrm{ng} \mathrm{g}^{-1} \mathrm{dw}$ ) were found in the soft tissue of Moluchia strigata collected in the San Ignacio-Navachiste-Macapule lagoon complex. The lowest concentrations $\left(<0.01 \mathrm{ng} \mathrm{g}^{-1} \mathrm{dw}\right)$ were found in C. californiensis collected in the lagoons Altata-Ensenada del Pabellon and Santa María-La Reforma.

In the case of freshwater mollusks, Corbicula sp. collected in the Colorado River had average concentrations of 350-1050 $\mathrm{ng} \mathrm{g}^{-1} \mathrm{dw}$. The highest $\sum$ DDTs $\quad\left(50-300 \quad \mathrm{ng}^{-1} \quad \mathrm{dw}\right)$ concentrations in crustaceans were found in $P$. clarkii collected at different sites and the Colorado River system. GarcíaHernández et al. (2015) conducted a biomonitoring study of POCs in several coastal lagoons of southern
Sonora and northern Sinaloa, using $C$. bellicosus biomonitor. They found average concentrations of $<0.01-180 \mathrm{ng} \mathrm{g}^{-1} \mathrm{dw}$ of $\sum$ DDTs, $<0.01-355 \mathrm{ng} \mathrm{g}^{-1} \mathrm{dw}$ of $\sum \mathrm{HCHs},<0.01-405 \mathrm{ng} \mathrm{g}^{-1} \mathrm{dw}$ of $\sum$ Drines, $<0.01$ $1035 \mathrm{ng} \mathrm{g}^{-1} \mathrm{dw}$ of $\sum$ Endosulfans, and $<0.01-310 \mathrm{ng} \mathrm{g}^{-1}$ $\mathrm{dw}$ of $\sum$ Heptachlor. The authors point out that these concentrations are relatively high and may be associated with the intense agricultural activity in the area and pesticides to control infectious vectors.

In the case of fish, the species associated with sediments showed high concentrations of POCs. UrestiMarín et al. (2008), for example, found average $\sum D D T$ concentrations of 1060 and $1245 \mathrm{ng} \mathrm{g}^{-1} \mathrm{dw}$ in muscle tissue of Ictalurus punctatus and Cyprinus carpio collected in the Vicente Guerrero Dam (Chihuahua), respectively. Similarly, García-Hernández et al. (2001) reported average whole-body concentrations of $875 \mathrm{ng}$ $\mathrm{g}^{-1} \mathrm{dw}$ of $\sum$ DDTs in I. punctatus collected in the Colorado River area. Furthermore, Reyes-Montiel et al. (2013) found average $\Sigma \mathrm{HCH}$ concentrations of $1015 \mathrm{ng}$ $\mathrm{g}^{-1} \mathrm{dw}$ in muscle tissue of $M$. cephalus from the San Ignacio-Navachiste-Macapule lagoon system. There were very few cases showing the behavior of POCs in the predator-prey relationship. In muscle tissue of $M$. cephalus, Reyes-Montiel et al. (2013) found average concentrations of $155 \mathrm{ng} \mathrm{g}^{-1} \mathrm{dw}$ of $\sum$ DDTs, $625 \mathrm{ng} \mathrm{g}^{-1}$ $\mathrm{dw}$ of $\sum$ Drines, $215 \mathrm{ng} \mathrm{g}^{-1} \mathrm{dw}$ of $\sum$ Endosulfans, and 75 $n g \mathrm{~g}^{-1} \mathrm{dw}$ of $\sum$ Heptachlor. Granados-Galván et al. (2015) reported average concentrations of $<0.01-50 \mathrm{ng}$ $\mathrm{g}^{-1} \mathrm{dw}$ of $\sum$ DDTs, $115-190 \mathrm{ng} \mathrm{g}^{-1} \mathrm{dw}$ of $\sum$ HCHs, 15-20 $\mathrm{ng} \mathrm{g}^{-1} \mathrm{dw}$ of $\sum$ Drines, $45-75 \mathrm{ng} \mathrm{g}^{-1} \mathrm{dw}$ of $\sum$ Endosulfans, and 30-40 $\mathrm{ng} \mathrm{g}^{-1} \mathrm{dw}$ of $\sum$ Heptachlor in three species of snappers. Both studies were conducted in the San Ignacio-Navachiste-Macapule lagoon system in the period 2011-2013.No biomagnification was observed, which may be related to the different detoxification rates of the various species. Moreover, low concentrations of POCs were observed in large pelagic fish, including sharks.

In marine mammals, a large amount of adipose tissue in them, their large size, longevity, and high trophic level allows them to bioaccumulate huge POCs amounts. Gallo-Reinoso et al. (2014), for example, found maximum concentrations of 87,300 $\mathrm{ng} \mathrm{g}^{-1} \mathrm{lw}$ (lipid weight) in biopsies of Delphinus capensis, while Niño-Torres et al. (2009) found maximum concentrations of $11,000 \mathrm{ng} \mathrm{g}^{-1} \mathrm{lw}$ in a biopsy of Zalophus californianus. The transfer of POCs via the mother became evident in sea turtles and aquatic birds. Average concentrations of 12-61 $\mathrm{ng} \mathrm{g}^{-1} \mathrm{ww}$ were found in seabird eggs collected throughout the Mexican Pacific. Carvalho et al. (2002) found higher levels of $\sum$ DDTs, $\sum$ HCHs, $\sum$ Drines, and $\sum$ Endosulfans in eggs in the muscle and liver tissue of Phalacrocorax sp. 
Table 3. Average concentration of POCs ( $\mathrm{ng} \mathrm{g}^{-1} \mathrm{dw}, \mathrm{D}$ or ww, $\mathrm{W}$ or $\mathrm{LW}, \mathrm{L}$ ) in different tissues of organisms from various aquatic ecosystems in Mexico. UR: Estero Urías; AEP: Altata- Ensenada del Pabellón; GCFR: Golfo de California; BGY: Bahía Guaymas; GSNR Granjas de Sonora; GSNL: Granjas de Sinaloa; INM: Laguna San Ignacio-Navachiste-Macapule; BCT: Bahía de Cueta; BBCN: Bocana; LTM: Laguna de Términos; SMR: Santa María- LA Reforma; PNGC: Parte Norte del Golfo de California; PXN: Punta Xen; LSI: Laguna de San Ignacio; PAO: Punta Abreojos; LAV: Laguna Alvarado; LVT: Laguna Vega de La Torre; BAP: Laguna Agiabampo; BOH: Bahía de Ohuria; CSC: Cienega Santa Clara; BMGD: Bahía Magdalena; SCY: Sacubay; CLSNR: Canal Sonora; DRC: Delta del Río Colorado; LTSC: Laguna de Terminos Sistema Candelaria-Panlau; LSCL: Laguna San Cristóbal; LMD: Laguna Madre; EPR: Estero Pozo del Rey; BYV: Laguna de Yavaros; LGVT: Laguna Grande- Laguna Vega de La Torre; BPZ: Bahía La Paz; ERCZ: estero del Rio Coatzacoalcos; RCL: Río Colorado; LCTC: Lago de Catemaco; SGJ: San Jorge; SPMT: San Pedro Mártir; SIF: San Ildefonso; IISB: Isla Isabel; IMT: Isla Marieta; ICND: Islas Coronado; BLB: Bahía de Lobos; RMYR: El Mayor; ZCO: Zona Costera de Oaxaca; IPR: Isla Pajarera; IPB: Isla Peña Blanca; CCP: Cucapa; LMCN: Lago Mechoacán; LMTT: Lago Metztitlán; IMP: Morro El Postosí; LTMH: Laguna de Tamiahua; LMCH: Laguna de la Mancha; LMXT: Laguna de Mexcaltitlan; XNH: Xnoha; MCU: Mocu; CDOC: El Doctor; CHPTN: Río Champoton-Los Petenes; PVG: Presa Vicente Guerrero; PAL: Presa Agua Limpia; RHD: Rio Hardy; LMDG: Laguna Mandinga.

DG05: Díaz-González et al. (2005); C02: Carvalho et al. (2002); GB07: Gold-Bouchot et al. (2007); C09: Carvalho et al. (2009); OL09: Osuna-López et al. (2009); CC11: Castañeda-Chavez et al. (2011); LR13: Lango-Reynoso et al. (2013); VB14: Vazquez-Boucard et al. (2014); GH01: Garcia-Hernández et al. (2001); VG16: Vargas-Gonzales et al. (2016); GH15: García-Hernández et al. (2015); GM10: Gonzalez-Mille et al. (2010); ER12: Espinosa-Reyes et al. (2012); RM13: Reyes-Montiel et al. (2013); GG15: Granados-Galván et al. (2015); MV14: Martínez-Villa et al. (2014); FB08: FernándezBringas et al. (2008); UM08: Uresti-Marín et al. (2008); AZ11: Aranguré-Zúniga et al. (2011); HG16: Hinojosa-Garro et al. (2016); GH13: García-Hernández et al. (2013); CV01: Calderon-Villagomez et al. (2001); G02: Gardner et al. (2003); LM11: Labrada-Martagón et al. (2011); GB14: García-Besné et al. (2014); GH06: García-Hernández et al. (2006); M09: Mellink et al. (2009); RR-RE11: Rivera-Rodríguez \& Rodríguez-Estrella (2011); GR14: Gallo-Reinoso et al. (2014); NT07: Niño-Torres et al. (2009); GJ12: González-Jauregui et al. (2012); RM06: Robledo-Marenco et al. (2006); C09: Carvalho et al. (2009). SON: Sonora; SIN: Sinaloa; NAY: Nayarit; JAL: Jalisco; COL: Colima; MIC: Michoacán; GRO: Guerrero; OAX: Oaxaca; CHP: Chiapas; CAM: Campeche; ROO: Quintana Roo; YUC: Yucatán; TAB: Tabasco; VER: Veracruz; TAM: Tamaulipas; BCS: Baja California Sur; BCN: Baja California Norte; AGU: Aguascalientes; MEX: Mexico; CMX: Ciudad de México. N/E: not specified; ND: not detect, -: not analized; D: dry weight; W: wet weight; M: muscle; ST: soft tissue; T: whole body; S: blood; B: biopsy; H: eggs.

¿DDTs: refers to the sum of Dichlorodiphenyltrichloroethane (DDT), DDE (dichlorodiphenyldichloroethylene), and DDD (dichlorodiphenyldichloroethane). $\sum$ HCHs: refers to the sum of Alpha-hexachlorocyclohexane $(\alpha-\mathrm{HCH})$, betahexachlorocyclohexane $(\beta-\mathrm{HCH})$, gamma-hexachlorocyclohexane $(\gamma-\mathrm{HCH})$, delta-hexachlorocyclohexane $(\delta-\mathrm{HCH})$. $\sum$ Drines: refers to the sum of Dialdrine, Aldrine, endrine, endrin aldehyde, endrin ketone. $\sum$ Endosulfans: refers to the sum of Endosulfan I, Endosulfan II, Endosulfan sulfate. $\sum$ Heptachlors: refers to the sum of Heptachlor, heptachor hepoxide.

\begin{tabular}{|c|c|c|c|c|c|c|c|}
\hline Species & Place & DDTs & $\mathrm{HCHs}$ & Drines & Endosulfanes & Heptacloros & Cite \\
\hline \multicolumn{8}{|l|}{ Primary producers } \\
\hline Ruppia sp. & LTSC (CAM) & $59.6^{\mathrm{D}}(1988-89)$ & $4.8^{\mathrm{D}}(1988-89)$ & $51.3^{\mathrm{D}}(1988-89)$ & ND (1988-89) & $2^{\mathrm{D}}(1988-89)$ & DG05 \\
\hline Nimphaea sp. & LTSC (CAM) & $19.7^{\mathrm{D}}(1988-89)$ & $30^{\mathrm{D}}(1988-89)$ & $313^{\mathrm{D}}(1988-89)$ & ND (1988-89) & $90^{\mathrm{D}}(1988-89)$ & DG05 \\
\hline Halodule sp. & LTSC (CAM) & $193^{\mathrm{D}}(1988-89)$ & $88^{\mathrm{D}}(1988-89)$ & $1009^{\mathrm{D}}(1988-89)$ & $129^{\mathrm{D}}(1988-89)$ & $18^{\mathrm{D}}(1988-89)$ & DG05 \\
\hline \multicolumn{8}{|l|}{ Mollusks } \\
\hline \multirow{7}{*}{ Mytella strigata } & AEP (SIN) & $32.4^{\mathrm{D}}(1989) \mathrm{ST}$ & $2.9^{\mathrm{D}}(1989) \mathrm{ST}$ & $13.6^{\mathrm{D}}$ (1989) ST & - & $0.07 \mathrm{D}$ (1989) ST & $\mathrm{C} 02$ \\
\hline & INM (SIN) & $282^{\mathrm{D}}$ (1996) ST & - & - & - & - & OL09 \\
\hline & AEP SIN) & 99D (1996) ST & - & - & - & - & OL09 \\
\hline & BCT SON) & $147^{\mathrm{D}}$ (1996) ST & - & - & - & - & OL09 \\
\hline & UR (SIN) & $12^{\mathrm{D}}$ (1996) ST & - & - & - & - & OL09 \\
\hline & LMXT (NAY) & $4.8^{\mathrm{D}}(1996) \mathrm{ST}$ & - & - & - & - & OL09 \\
\hline & LSCL (NAY) & $69^{\mathrm{D}}$ (1996) ST & - & - & - & - & OL09 \\
\hline \multirow{8}{*}{ Crassostrea virginica } & LTM (CAM) & $9.1^{\mathrm{D}}(2002) \mathrm{ST}$ & $16.3^{\mathrm{D}}(2002) \mathrm{ST}$ & $22.4^{\mathrm{D}}(2002) \mathrm{ST}$ & - & - & GB07 \\
\hline & LTMH (VER) & $114^{\mathrm{L}}(2005) \mathrm{ST}$ & - & - & - & - & $\mathrm{CC} 11$ \\
\hline & LVT (VER) & $103^{\mathrm{L}}(2005) \mathrm{ST}$ & - & - & - & - & $\mathrm{CC} 11$ \\
\hline & LAV (VER) & $53^{\mathrm{L}}(2001-02) \mathrm{ST}$ & - & - & - & - & $\mathrm{CC} 11$ \\
\hline & LMCH VER) & $99^{\mathrm{L}}(2001-02) \mathrm{ST}$ & - & - & - & - & $\mathrm{CC} 11$ \\
\hline & LMD (TAM) & $22^{\mathrm{L}}(2008) \mathrm{ST}$ & 63L (2008) ST & $355^{\mathrm{L}}(2008) \mathrm{ST}$ & $13^{\mathrm{L}}(2008) \mathrm{ST}$ & - & LR13 \\
\hline & LMDG (VER) & $89^{\mathrm{L}}(2008) \mathrm{ST}$ & $1614^{\mathrm{L}}(2008) \mathrm{ST}$ & $347^{\mathrm{L}}(2008) \mathrm{ST}$ & $136^{\mathrm{L}}(2008) \mathrm{ST}$ & - & LR13 \\
\hline & LMCN (TAB) & $20^{\mathrm{L}}(2009) \mathrm{ST}$ & $23^{\mathrm{L}}(2008) \mathrm{ST}$ & $57^{\mathrm{L}}(2008) \mathrm{ST}$ & $14^{\mathrm{L}}(2008) \mathrm{ST}$ & - & LR13 \\
\hline \multirow[t]{3}{*}{ Crassostrea gigas } & AEP (SIN) & $29^{\mathrm{D}}$ (1989) ST & $0.7^{\mathrm{D}}(1989) \mathrm{ST}$ & $18.9^{\mathrm{D}}(1989) \mathrm{ST}$ & - & - & $\mathrm{C} 02$ \\
\hline & GSNR (SON) & 3D (N/E) ST & $72^{\mathrm{D}}$ (N/E) ST & - & - & - & VB14 \\
\hline & GSNL (SIN) & $1.1^{\mathrm{D}}(\mathrm{N} / \mathrm{E}) \mathrm{ST}$ & $44^{\mathrm{D}}$ (N/E) ST & - & - & - & VB14 \\
\hline Crassostrea sp. & LTM (CAM) & $5.8^{\mathrm{D}}(\mathrm{N} / \mathrm{E}) \mathrm{ST}$ & $1.4^{\mathrm{D}}(\mathrm{N} / \mathrm{E}) \mathrm{ST}$ & $0.2^{\mathrm{D}}(\mathrm{N} / \mathrm{E}) \mathrm{ST}$ & $0.38^{\mathrm{D}}(\mathrm{N} / \mathrm{E}) \mathrm{ST}$ & $0.08^{\mathrm{D}}(\mathrm{N} / \mathrm{E}) \mathrm{ST}$ & $\mathrm{C} 09$ \\
\hline Chione sp. & PNGC (SON) & ND (1998-00) ST & - & - & - & - & GH01 \\
\hline Corbicula sp. & $\mathrm{RCL}(\mathrm{SON})$ & $350^{\mathrm{D}}(1998-00) \mathrm{ST}$ & - & - & - & - & GH01 \\
\hline
\end{tabular}


continuation

\begin{tabular}{|c|c|c|c|c|c|c|c|}
\hline Species & Place & DDTs & $\mathrm{HCHs}$ & Drines & Endosulfanes & Heptacloros & Cite \\
\hline Corbicula sp. & CLSNR (SON) & $1050^{\mathrm{D}}(1998-00) \mathrm{ST}$ & - & - & - & - & GH01 \\
\hline Chione californiensis & BYV (SON) & $73^{\mathrm{D}}(2009) \mathrm{ST}$ & ND (2009) ST & $33^{\mathrm{D}}$ (2009) ST & ND (2009) ST & $63^{\mathrm{D}}(2009) \mathrm{ST}$ & VG16 \\
\hline Chione californiensis & $\mathrm{AEP}(\mathrm{SIN})$ & ND (2009) ST & ND (2009) ST & 7D (2009) ST & ND (2009) ST & $8.19^{\mathrm{D}}(2009) \mathrm{ST}$ & VG16 \\
\hline Chione californiensis & SMR (SIN) & ND (2009) ST & ND (2009) ST & ND (2009) ST & ND (2009) ST & ND (2009) ST & VG16 \\
\hline Chione subrogosa & AEP (SIN) & $2.3^{\mathrm{D}}(1989) \mathrm{ST}$ & $1.2^{\mathrm{D}}(1989) \mathrm{ST}$ & $7.5^{\mathrm{D}}$ (1989) ST & $32.2^{\mathrm{D}}(1989) \mathrm{ST}$ & ND (1989) ST & $\mathrm{C} 02$ \\
\hline Pomacea patula & LCTC (VER) & ND (1993) M & ND (1993) M & $96.5^{\mathrm{D}}(1993) \mathrm{M}$ & - & - & CV01 \\
\hline \multicolumn{8}{|l|}{ Crustaceans } \\
\hline Penaeus vannamei & $\operatorname{AEP}(\mathrm{SIN})$ & $1.1^{\mathrm{D}}(1989) \mathrm{M}$ & ND (1989) M & $1.1^{\mathrm{D}}(1989) \mathrm{M}$ & $3^{\mathrm{D}}(1989) \mathrm{M}$ & ND (1989) M & $\mathrm{C} 02$ \\
\hline Penaeus stylirostri & $\mathrm{AEP}(\mathrm{SIN})$ & $6.6^{\mathrm{D}}(1991) \mathrm{M}$ & $1.3^{\mathrm{D}}(1991) \mathrm{M}$ & $1^{\mathrm{D}}(1991) \mathrm{M}$ & $0.7^{\mathrm{D}}(1991) \mathrm{M}$ & - & $\mathrm{C} 02$ \\
\hline Penaeus sp. & BOH (SIN) & $0.02^{\mathrm{D}}(1995-96) \mathrm{M}$ & $0.17^{\mathrm{D}}(1995-96) \mathrm{M}$ & - & $1.06^{\mathrm{D}}(1995-96) \mathrm{M}$ & $0.14^{\mathrm{D}}(1995-96) \mathrm{M}$ & OF-R02 \\
\hline Litopenaeus sp. & EPR (NAY) & $26^{\mathrm{D}}(1996-97) \mathrm{M}$ & $21^{\mathrm{D}}(1996-97) \mathrm{M}$ & $28^{\mathrm{D}}(1996-97) \mathrm{M}$ & $25^{\mathrm{D}}(1996-97) \mathrm{M}$ & $18^{\mathrm{D}}(1996-97) \mathrm{M}$ & RM06 \\
\hline Litopenaeus sp. & LCSL (NAY) & $26^{\mathrm{D}}(1996-97) \mathrm{M}$ & $42^{\mathrm{D}}(1996-97) \mathrm{M}$ & $4.6^{\mathrm{D}}(1996-97) \mathrm{M}$ & $45^{\mathrm{D}}(1996-97) \mathrm{M}$ & $0.01^{\mathrm{D}}(1996-97) \mathrm{M}$ & RM06 \\
\hline Callinectes sp. & ERCZ (VER) & $2.5^{\mathrm{D}}(2006) \mathrm{M}$ & $15^{\mathrm{D}}(2006) \mathrm{M}$ & - & - & - & ER12 \\
\hline \multirow{4}{*}{ Callinectes bellicosus } & BYV (SON) & ND (2012) M & $355^{\mathrm{D}}(2012) \mathrm{M}$ & $405^{\mathrm{D}}(2012) \mathrm{M}$ & $340^{\mathrm{D}}(2012) \mathrm{M}$ & ND (2012) M & GH15 \\
\hline & $\mathrm{BAP}(\mathrm{SON})$ & $180^{\mathrm{D}}(2012) \mathrm{M}$ & ND (2012) M & $425^{\mathrm{D}}(2012) \mathrm{M}$ & $855^{\mathrm{D}}(2012) \mathrm{M}$ & $130^{\mathrm{D}}(2012) \mathrm{M}$ & GH15 \\
\hline & BLB (SON) & ND (2012) M & $195^{\mathrm{D}}(2012) \mathrm{M}$ & ND (2012) M & ND (2012) M & $310^{\mathrm{D}}(2012) \mathrm{M}$ & GH15 \\
\hline & BOH (SIN) & ND (2012) M & $41^{\mathrm{D}}(2012) \mathrm{M}$ & ND (2012) M & $1035^{\mathrm{D}}(2012) \mathrm{M}$ & $100^{\mathrm{D}}(2012) \mathrm{M}$ & GH15 \\
\hline Callinectes rathbunae & LGVT (VER) & $267^{\mathrm{L}}(2008) \mathrm{M}$ & - & - & - & - & LR13 \\
\hline \multirow[t]{3}{*}{ Procambarus clarki } & RMYR (SON) & $135^{\mathrm{D}}(1998-00) \mathrm{T}$ & - & - & - & - & GH01 \\
\hline & $\mathrm{CCP}(\mathrm{SON}$ & $300^{\mathrm{D}}(1998-00) \mathrm{T}$ & - & - & - & - & GH01 \\
\hline & $\mathrm{CSC}(\mathrm{SON})$ & $50^{\mathrm{D}}(1998-00) \mathrm{T}$ & - & - & - & - & GH01 \\
\hline Paleomonetes paludosus & $\mathrm{BBCN}(\mathrm{SON})$ & ND $(1998-00) \mathrm{T}$ & - & - & - & - & GH01 \\
\hline \multirow{6}{*}{ Mugil cephalus } & & & Fishes & & & & \\
\hline & AEP (SIN) & $20^{\mathrm{D}}(1991) \mathrm{M}$ & $3.5^{\mathrm{D}}(1991) \mathrm{M}$ & $1.4^{\mathrm{D}}(1991) \mathrm{M}$ & $4.6^{\mathrm{D}}(1991) \mathrm{M}$ & ND (1991) M & $\mathrm{C} 02$ \\
\hline & ERCZ (VER) & $0.98^{\mathrm{D}}(2006) \mathrm{M}$ & $1.6^{\mathrm{D}}(2006) \mathrm{M}$ & - & - & - & GM10 \\
\hline & ERCZ (VER) & $1^{\mathrm{D}}(2006) \mathrm{M}$ & $2.5^{\mathrm{D}}(2006) \mathrm{M}$ & - & - & - & ER12 \\
\hline & INM (SIN) & $155^{\mathrm{D}}(2010-11) \mathrm{M}$ & $1015^{\mathrm{D}}(2010-11) \mathrm{M}$ & $625^{\mathrm{D}}(2010-11) \mathrm{M}$ & $215^{\mathrm{D}}(2010-11) \mathrm{M}$ & $75^{\mathrm{D}}(2010-11) \mathrm{M}$ & RM13 \\
\hline & $\mathrm{CSC}(\mathrm{SON})$ & $250^{\mathrm{D}}(1998-00) \mathrm{T}$ & - & - & - & - & GH01 \\
\hline Arius melanopus & LTSC (CAM) & $116^{\mathrm{D}}(1999) \mathrm{M}$ & $26^{\mathrm{D}}(1999) \mathrm{M}$ & $509^{\mathrm{D}}(1999) \mathrm{M}$ & 49D (1999) M & $300^{\mathrm{D}}(1999) \mathrm{M}$ & DG05 \\
\hline Lepisosteus tropicus & LTM (CAM) & $15^{\mathrm{D}}(\mathrm{N} / \mathrm{E}) \mathrm{M}$ & - & - & - & - & C09 \\
\hline \multirow[t]{3}{*}{ Cyprinus carpio } & PAL (NAY) & $1.9^{\mathrm{D}}(2004) \mathrm{M}$ & ND (2004) M & $7.1^{\mathrm{D}}(2004) \mathrm{M}$ & $1.5^{\mathrm{D}}(2004) \mathrm{M}$ & ND (2004) M & AZ11 \\
\hline & $\mathrm{CSC}(\mathrm{SON})$ & $275^{\mathrm{D}}(1998-00) \mathrm{T}$ & - & - & - & - & GH01 \\
\hline & PVG (CHH) & $1245^{\mathrm{D}}(2004) \mathrm{M}$ & - & $27^{\mathrm{D}}(2004) \mathrm{M}$ & - & ND (2004) M & UM08 \\
\hline \multirow{2}{*}{ Eugerris axillaris } & ERCZ (VER) & $0.5^{\mathrm{D}}(2006) \mathrm{M}$ & $11^{\mathrm{D}}(2006) \mathrm{M}$ & - & - & - & ER12 \\
\hline & ERCZ (VER) & $0.49^{\mathrm{D}}(2006) \mathrm{M}$ & $12.8^{\mathrm{D}}(2006) \mathrm{M}$ & - & - & - & GM10 \\
\hline Oreochromis aureus & PAL (NAY) & $0.5^{\mathrm{D}}(2004) \mathrm{M}$ & ND (2004) M & $3^{\mathrm{D}}(2004) \mathrm{M}$ & $4.3^{\mathrm{D}}(2004) \mathrm{M}$ & ND (2004) M & AZ11 \\
\hline \multirow[t]{2}{*}{ Oreochromis niloticus } & XNH (CAM) & $16739^{\mathrm{L}}(2011) \mathrm{M}$ & $335^{\mathrm{L}}(2011) \mathrm{M}$ & $473^{\mathrm{L}}(2011) \mathrm{M}$ & $376^{\mathrm{L}}(2011) \mathrm{M}$ & - & HG16 \\
\hline & PVG (CHH) & $155^{\mathrm{D}}(2004) \mathrm{M}$ & - & $30.5^{\mathrm{D}}(2004) \mathrm{M}$ & - & ND (2004) M & UM08 \\
\hline \multirow[t]{4}{*}{ Oreochromis sp. } & ERCZ (VER) & $1^{\mathrm{D}}(2006) \mathrm{M}$ & $1.5^{\mathrm{D}}(2006) \mathrm{M}$ & - & - & - & ER12 \\
\hline & ERCZ (VER) & $0.99^{\mathrm{D}}(2006) \mathrm{M}$ & $2^{\mathrm{D}}(2006) \mathrm{M}$ & - & - & - & GM10 \\
\hline & LMTT (HID) & $4^{\mathrm{D}}(2001) \mathrm{M}$ & $20^{\mathrm{D}}(2001) \mathrm{M}$ & $0.5^{\mathrm{D}}(2001) \mathrm{M}$ & $6^{\mathrm{D}}(2001) \mathrm{M}$ & $1.5^{\mathrm{D}}(2001) \mathrm{M}$ & FB08 \\
\hline & LMTT (HID) & $4^{\mathrm{D}}(2002) \mathrm{M}$ & $1.2^{\mathrm{D}}(2002) \mathrm{M}$ & $1.5^{\mathrm{D}}(2002) \mathrm{M}$ & $1.5^{\mathrm{D}}(2002) \mathrm{M}$ & $43^{\mathrm{D}}(2002) \mathrm{M}$ & FB08 \\
\hline \multirow[t]{2}{*}{ Centropomus parallelus } & ERCZ (VER) & $0.5^{\mathrm{D}}(2006) \mathrm{M}$ & $6.5^{\mathrm{D}}(2006) \mathrm{M}$ & - & - & - & ER12 \\
\hline & ERCZ (VER) & $0.5^{\mathrm{D}}(2006) \mathrm{M}$ & $7.5^{\mathrm{D}}(2006) \mathrm{M}$ & - & - & - & GM10 \\
\hline \multirow[t]{2}{*}{ Arius felis } & ERCZ (VER) & $1^{\mathrm{D}}(2006) \mathrm{M}$ & $2^{\mathrm{D}}(2006) \mathrm{M}$ & - & - & - & ER12 \\
\hline & ERCZ (VER) & $0.99^{\mathrm{D}}(2006) \mathrm{M}$ & $1.9^{\mathrm{D}}(2006) \mathrm{M}$ & - & - & - & GM10 \\
\hline \multirow[t]{2}{*}{ Poecilia latipinna } & RCL (SON) & $1300^{\mathrm{D}}(1998-00) \mathrm{T}$ & - & - & - & - & GH01 \\
\hline & $\mathrm{CSC}(\mathrm{SON})$ & $1350^{\mathrm{D}}(1998-00) \mathrm{T}$ & - & - & - & - & GH01 \\
\hline Ictalurus punctatus & RHD (SON) & $100^{\mathrm{D}}(1998-00) \mathrm{T}$ & - & - & - & - & GH01 \\
\hline & $\mathrm{CCP}(\mathrm{SON})$ & $875^{\mathrm{D}}(1998-00) \mathrm{T}$ & - & - & - & - & GH01 \\
\hline & PVG (CHH) & $1060^{\mathrm{D}}(2004) \mathrm{M}$ & - & $16.5^{\mathrm{D}}(2004) \mathrm{M}$ & - & ND (2004) M & UM08 \\
\hline & PAL (NAY) & ND (2004) M & $3.8^{\mathrm{D}}(2004) \mathrm{M}$ & $7.6^{\mathrm{D}}(2004) \mathrm{M}$ & $0.18^{\mathrm{D}}(2004) \mathrm{M}$ & ND (2004) M & AZ11 \\
\hline Micropterus salmoides & PAL (NAY) & ND (2004) M & $0.1^{\mathrm{D}}(2004) \mathrm{M}$ & $3.6^{\mathrm{D}}(2004) \mathrm{M}$ & $3.5^{\mathrm{D}}(2004) \mathrm{M}$ & $0.5^{\mathrm{D}}(2004) \mathrm{M}$ & AZ11 \\
\hline & PVG $(\mathrm{CHH})$ & $865^{\mathrm{D}}(2004) \mathrm{M}$ & - & $22.5^{\mathrm{D}}(2004) \mathrm{M}$ & - & $60^{\mathrm{D}}(2004) \mathrm{M}$ & UM08 \\
\hline Gambusia affinis & RMYR (SON) & $1600^{\mathrm{D}}(1998-00) \mathrm{T}$ & - & - & - & - & GH01 \\
\hline & $\mathrm{CSC}(\mathrm{SON})$ & $300^{\mathrm{D}}(1998-00) \mathrm{T}$ & - & - & - & - & GH01 \\
\hline & CDOC (SON) & $850^{\mathrm{D}}(1998-00) \mathrm{T}$ & - & - & - & - & GH01 \\
\hline Dorosoma petenense & LCTC (VER) & $6.5^{\mathrm{D}}(1993) \mathrm{M}$ & ND (1993) M & $33.5^{\mathrm{D}}(1993) \mathrm{M}$ & - & - & CV01 \\
\hline Cichlasoma urophthalmus & MCU (CAM) & $19047^{L}$ (2011) M & $442^{\mathrm{L}}(2011) \mathrm{M}$ & $521^{\mathrm{L}}(2011) \mathrm{M}$ & $405^{\mathrm{L}}(2011) \mathrm{M}$ & - & HG16 \\
\hline Lepomis macrochirus & RHD (SON) & $310^{\mathrm{D}}(2010) \mathrm{M}$ & DL (2010) M & $60^{\mathrm{D}}(2010) \mathrm{M}$ & ND (2010) M & ND (2010) M & GH13 \\
\hline Lutjanus argentiventris & INM (SIN) & $40^{\mathrm{D}}(2012-13) \mathrm{M}$ & $120^{\mathrm{D}}(2012-13) \mathrm{M}$ & $15^{\mathrm{D}}(2013-13) \mathrm{M}$ & $50^{\mathrm{D}}(2013-13) \mathrm{M}$ & $40^{\mathrm{D}}(2013-13) \mathrm{M}$ & GG15 \\
\hline Lutjanus novemfasciatus & INM (SIN) & ND (2012-13) M & $190^{\mathrm{D}}(2012-13) \mathrm{M}$ & $15^{\mathrm{D}}(2013-13) \mathrm{M}$ & $45^{\mathrm{D}}(2013-13) \mathrm{M}$ & $30^{\mathrm{D}}(2013-13) \mathrm{M}$ & GG15 \\
\hline Lutjanus colorado & INM (SIN) & $50^{\mathrm{D}}(2012-13) \mathrm{M}$ & $115^{\mathrm{D}}(2012-13) \mathrm{M}$ & $20^{\mathrm{D}}(2013-13) \mathrm{M}$ & 75 $(2013-13) \mathrm{M}$ & $40^{\mathrm{D}}(2013-13) \mathrm{M}$ & GG15 \\
\hline Euthynnus lineatus & $\mathrm{ZCO}(\mathrm{OAX})$ & $31^{\mathrm{D}}(2010-11) \mathrm{M}$ & $16.5^{\mathrm{D}}(2010-11) \mathrm{M}$ & $18^{\mathrm{D}}(2010-11) \mathrm{M}$ & $43^{\mathrm{D}}(2010-11) \mathrm{M}$ & $15^{\mathrm{D}}(2010-11) \mathrm{M}$ & MV14 \\
\hline Thunnus albacares & ZCO (OAX) & $22.5^{\mathrm{D}}(2010-11) \mathrm{M}$ & $7.5^{\mathrm{D}}(2010-11) \mathrm{M}$ & ND (2010-11) M & $30.5^{\mathrm{D}}(2010-11) \mathrm{M}$ & $14^{\mathrm{D}}(2010-11) \mathrm{M}$ & MV14 \\
\hline Sarda orientalis & ZCO (OAX) & $6^{\mathrm{D}}(2010-11) \mathrm{M}$ & ND (2010-11) M & $9.5^{\mathrm{D}}(2010-11) \mathrm{M}$ & ND (2010-2011) M & $12.5^{\mathrm{D}}(2010-11) \mathrm{M}$ & MV14 \\
\hline Coryphaena hippurus & $\mathrm{ZCO}(\mathrm{OAX})$ & $60^{\mathrm{D}}(2010-11) \mathrm{M}$ & ND (2010-11) M & $18^{\mathrm{D}}(2010-11) \mathrm{M}$ & 55 & $14^{\mathrm{D}}(2010-11) \mathrm{M}$ & MV14 \\
\hline
\end{tabular}


continuation

\begin{tabular}{|c|c|c|c|c|c|c|c|}
\hline Species & Place & DDTs & $\mathrm{HCHs}$ & Drines & Endosulfanes & Heptacloros & Cite \\
\hline \multicolumn{8}{|l|}{ Sharks } \\
\hline Sphyrna lewini & $\mathrm{ZCO}(\mathrm{OAX})$ & $4.4^{\mathrm{D}}(2010-11) \mathrm{M}$ & $5.6^{\mathrm{D}}(2010-11) \mathrm{M}$ & $5.6^{\mathrm{D}}(2010-11) \mathrm{M}$ & ND (2010-11) M & $16^{\mathrm{D}}(2010-11) \mathrm{M}$ & MV14 \\
\hline Carcharhinus leucas & $\mathrm{ZCO}(\mathrm{OAX})$ & $9.6^{\mathrm{D}}(2010-11) \mathrm{M}$ & $8.4^{\mathrm{D}}(2010-11) \mathrm{M}$ & $4.5^{\mathrm{D}}(2010-11) \mathrm{M}$ & ND $(2010-11) \mathrm{M}$ & $6^{\mathrm{D}}(2010-11) \mathrm{M}$ & MV14 \\
\hline \multicolumn{8}{|l|}{ Turtles } \\
\hline \multirow[t]{5}{*}{ Chelonia mydas } & BPZ (BCS) & $7^{\mathrm{w}}(\mathrm{N} / \mathrm{E}) \mathrm{M}$ & $4.8^{\mathrm{w}}(\mathrm{N} / \mathrm{E}) \mathrm{M}$ & $2^{\mathrm{W}}(\mathrm{N} / \mathrm{E}) \mathrm{M}$ & $3.8^{\mathrm{w}}(\mathrm{N} / \mathrm{E}) \mathrm{M}$ & - & G02 \\
\hline & PAO (BCS) & $0.7^{\mathrm{W}}(2005-07) \mathrm{S}$ & $2.8^{\mathrm{W}}(2005-07) \mathrm{S}$ & $3.4^{\mathrm{W}}(2005-07) \mathrm{S}$ & ND (2005-07) S & $8.6^{\mathrm{W}}(2005-07) \mathrm{S}$ & LM1 1 \\
\hline & BMGD (BCS) & ND (2005-07) S & $11^{\mathrm{w}}(2005-07) \mathrm{S}$ & $10^{\mathrm{W}}(2005-07) \mathrm{S}$ & ND (2005-07) S & $4.5^{\mathrm{w}}(2005-07) \mathrm{S}$ & LM1 1 \\
\hline & PXN (CAM) & $14^{\mathrm{L}}(\mathrm{N} / \mathrm{E}) \mathrm{S}$ & $4.3^{\mathrm{L}}(\mathrm{N} / \mathrm{E}) \mathrm{S}$ & $17^{\mathrm{L}}(\mathrm{N} / \mathrm{E}) \mathrm{S}$ & $12^{\mathrm{L}}(\mathrm{N} / \mathrm{E}) \mathrm{S}$ & $2^{\mathrm{L}}(\mathrm{N} / \mathrm{E}) \mathrm{S}$ & GB14 \\
\hline & PXN (CAM) & $729^{\mathrm{L}}(\mathrm{N} / \mathrm{E}) \mathrm{H}$ & $335^{\mathrm{L}}(\mathrm{N} / \mathrm{E}) \mathrm{H}$ & $1470^{\mathrm{L}}(\mathrm{N} / \mathrm{E}) \mathrm{H}$ & $672^{\mathrm{L}}(\mathrm{N} / \mathrm{E}) \mathrm{H}$ & $78^{\mathrm{L}}(\mathrm{N} / \mathrm{E}) \mathrm{H}$ & GB14 \\
\hline Lepidochelys olivacea & BPZ (BCS) & $8.6^{\mathrm{W}}(\mathrm{N} / \mathrm{E}) \mathrm{M}$ & $14^{\mathrm{W}}(\mathrm{N} / \mathrm{E}) \mathrm{M}$ & $3^{\mathrm{w}}(\mathrm{N} / \mathrm{E}) \mathrm{M}$ & $14^{\mathrm{W}}(\mathrm{N} / \mathrm{E}) \mathrm{M}$ & - & G02 \\
\hline Caretta caretta & BPZ BS) & $3^{\mathrm{W}}(\mathrm{N} / \mathrm{E}) \mathrm{M}$ & $3^{\mathrm{w}}(\mathrm{N} / \mathrm{E}) \mathrm{M}$ & $3^{\mathrm{W}}(\mathrm{N} / \mathrm{E}) \mathrm{M}$ & $3^{\mathrm{w}}(\mathrm{N} / \mathrm{E}) \mathrm{M}$ & - & G02 \\
\hline Eretmochelys imbricata & SCY (CAM) & $25^{\mathrm{L}}(\mathrm{N} / \mathrm{E}) \mathrm{S}$ & $6.6^{\mathrm{L}}(\mathrm{N} / \mathrm{E}) \mathrm{S}$ & $17^{\mathrm{L}}(\mathrm{N} / \mathrm{E}) \mathrm{S}$ & ${ }^{9 \mathrm{~L}}$ (N/E) $\mathrm{S}$ & $19^{\mathrm{L}}(\mathrm{N} / \mathrm{E}) \mathrm{S}$ & GB14 \\
\hline Eretmochelys imbricata & SCY (CAM) & $8066^{\mathrm{L}}(\mathrm{N} / \mathrm{E}) \mathrm{H}$ & $665^{\mathrm{L}}(\mathrm{N} / \mathrm{E}) \mathrm{H}$ & $4818^{\mathrm{L}}(\mathrm{N} / \mathrm{E}) \mathrm{H}$ & $1132^{\mathrm{L}}(\mathrm{N} / \mathrm{E}) \mathrm{H}$ & 1284L (N/E) H & GB14 \\
\hline \multicolumn{8}{|l|}{ Birds } \\
\hline Athene cunicularia & $\mathrm{DRC}(\mathrm{SON})$ & $48^{\mathrm{W}}(\mathrm{N} / \mathrm{E}) \mathrm{H}$ & $14^{\mathrm{W}}(\mathrm{N} / \mathrm{E}) \mathrm{H}$ & $0.5^{\mathrm{W}}(\mathrm{N} / \mathrm{E}) \mathrm{H}$ & - & $8.26^{\mathrm{w}}(\mathrm{N} / \mathrm{E}) \mathrm{H}$ & GH06 \\
\hline Zenaida macroura & DRC (SON) & $61^{\mathrm{W}}(\mathrm{N} / \mathrm{E}) \mathrm{H}$ & $32^{\mathrm{W}}(\mathrm{N} / \mathrm{E}) \mathrm{H}$ & $3.7^{\mathrm{W}}(\mathrm{N} / \mathrm{E}) \mathrm{E}$ & - & $2.7^{\mathrm{W}}(\mathrm{N} / \mathrm{E}) \mathrm{H}$ & GH06 \\
\hline \multirow{8}{*}{ Sula leucogaster } & SGJ (SON) & $53^{\mathrm{w}}(2006) \mathrm{H}$ & - & - & - & - & M09 \\
\hline & SPMT (SON) & $52^{\mathrm{W}}(2006) \mathrm{H}$ & - & - & - & - & M09 \\
\hline & SIF (SIN) & $34^{\mathrm{W}}(2006) \mathrm{H}$ & - & - & - & - & M09 \\
\hline & IISB (NAY) & $38^{\mathrm{w}}(2006) \mathrm{H}$ & - & - & - & - & M09 \\
\hline & IMT (JAL) & $49^{\mathrm{w}}(2006) \mathrm{H}$ & - & - & - & - & M09 \\
\hline & IPR (JAL) & $22^{\mathrm{W}}(2006) \mathrm{H}$ & - & - & - & - & M09 \\
\hline & IPB (COL) & $12^{\mathrm{W}}(2006) \mathrm{H}$ & - & - & - & - & M09 \\
\hline & IMP $(\mathrm{MCH})$ & $14^{\mathrm{W}}(2006) \mathrm{H}$ & - & - & - & - & M09 \\
\hline Pandion haliaetus & LSI (BCS) & $0.9^{w}(2001) S$ & $0.4^{\mathrm{W}}(2001) \mathrm{S}$ & $2.3^{\mathrm{W}}(2001) \mathrm{W}$ & $0.09^{\mathrm{W}}(2001) \mathrm{S}$ & $0.2^{\mathrm{W}}(2001) \mathrm{H}$ & RR-RE11 \\
\hline \multirow[t]{2}{*}{ Phalacrocorax sp. } & AEP (SIN) & 5824D (1991) M & $284^{\mathrm{D}}(1991) \mathrm{M}$ & $13.5^{\mathrm{D}}(1991) \mathrm{M}$ & $1.2^{\mathrm{D}}(1991) \mathrm{M}$ & - & $\mathrm{C} 02$ \\
\hline & AEP (SIN) & $12047^{\mathrm{D}}(1991) \mathrm{H}$ & $1200^{\mathrm{D}}(1991) \mathrm{H}$ & $45^{\mathrm{D}}(1991) \mathrm{H}$ & $1.1^{\mathrm{D}}(1991) \mathrm{H}$ & - & $\mathrm{C} 02$ \\
\hline \multicolumn{8}{|l|}{ Mammals } \\
\hline Delphinus capensis & BGY (SON) & $18000^{\mathrm{L}}(1999) \mathrm{B}$ & - & - & - & - & GR14 \\
\hline Zalophus californianus & GCFR (N/A) & $2750^{\mathrm{L}}(2005-06) \mathrm{B}$ & $35^{\mathrm{L}}(2005-06) \mathrm{B}$ & - & - & - & NT07 \\
\hline \multicolumn{8}{|l|}{ Crocodiles } \\
\hline Crocodylus moreletti & CHPTN (CAM) & $1800^{\mathrm{L}}(2005-06) \mathrm{S}$ & $550^{\mathrm{L}}(2005-06) \mathrm{S}$ & - & $\mathrm{ND}(2005-06) \mathrm{S}$ & - & GJ12 \\
\hline
\end{tabular}

García-Besné et al. (2014) reported concentrations of POCs hundreds of times higher in eggs of E. imbricata and $C$. mydas than in blood. However, long-term effects on the embryo exposure to these contaminants via the mother have not been thoroughly studied yet.

\section{Effect of PBTSs on aquatic organisms and human health}

The effect of acute exposure to many substances on different aquatic organisms (micro and macroinvertebrates, fish, reptiles, amphibians, birds, and mammals) has been well studied. Despite this, the effect of such exposure on wild organisms is not yet well known, mainly due to the technical complexity of the field experiments, and to the fact that, in the vast majority of cases, the concentrations in which the pollutants occur in different environmental matrices are barely noticeable, which means their effects are not observable in the short term. However, these compounds' bioaccumulation in different tissues reveals chronic exposure to wild organisms.

The concentration of contaminants in different tissues can be correlated with direct observations made in the field (biometric, reproductive and behavioral, among others), or compared with reference values for residual concentrations in tissue or tissue residue guidelines (TRG), which establish the maximum concentration (quantitatively) of a substance in different tissues (including food) that is recommended for wildlife protection (CCME, 1998). These TRGs are based on many field and laboratory experiments and aim to link measurements made in wild organisms with robust experimental toxicological information to predict the populations under study's potential risks.

This review shows the reported concentrations of the main PBTS in many aquatic species (Tables 1-3). Weng \& Wang (2017) found that Cd concentrations between 17-27 $\mu \mathrm{g} \mathrm{g}^{-1} \mathrm{dw}$ in Crassostrea hongkongensis were associated with low fertility rates and slow larval growth compared to organisms sampled at reference sites. In the present review, we found that, in $93 \%$ of cases, Cd's average concentration in bivalves was 0.2 $17 \mu \mathrm{g} \mathrm{g}^{-1} \mathrm{dw}$, reaching up to $22,28,36,42$, and $57 \mu \mathrm{g}$ $\mathrm{g}^{-1} \mathrm{dw}$ in particular sites and dates. Wiener et al. (2003) indicated that $\mathrm{Hg}$ concentrations of $6-20 \mu \mathrm{g} \mathrm{g}^{-1} \mathrm{ww}$ in muscle tissue of adult fish were associated with sublethal effects, including behavioral alterations, brain lesions, and deterioration of gonadal development.

All cases of $\mathrm{Hg}$ in fish muscle reviewed here were below this concentration. In birds, Ceyca et al. (2016) 
indicated that $\mathrm{Hg}$ concentrations of $0.5-1 \mu \mathrm{g} \mathrm{g}^{-1} \mathrm{ww}$ in sea bird eggs could cause mortality, malformations, and neurological damage. Ceyca et al. (2016) also reported maximum $\mathrm{Hg}$ values of $0.55-187 \mu \mathrm{g} \mathrm{g}^{-1} \mathrm{ww}$ in eight species of seabirds collected on the coast of Sinaloa. Zhang et al. (2013) reported that the lowest probable adverse effect (LOAEL) of $\mathrm{Hg}$ on aquatic birds' blood occurred at $0.73 \mu \mathrm{g} \mathrm{g}^{-1}$ ww. Lerma et al. (2016) reported $\mathrm{Hg}$ values of $1.1 \mathrm{ppm} \mathrm{dw}$ in the blood of Sula leucogaster collected on the coast of Sinaloa.

Niño-Torres et al. (2009) reported that DDT values $<980,000 \mu \mathrm{g} \mathrm{g}^{-1} \mathrm{lw}$ in fatty tissue of marine mammals were associated with premature deliveries, while PCB concentrations $<17 \mu \mathrm{g} \mathrm{g}^{-1} \mathrm{lw}$ in fatty tissue could cause endocrine disruption and immunosuppression. GalloReinoso et al. (2014) reported DDT concentrations of $21 \mu \mathrm{g} \mathrm{g}^{-1} \mathrm{lw}$ in the biopsy of a common dolphin sampled in the Gulf of California. Regarding TRGs, the Canadian Council of Ministers of the Environment (CCME) establishes a reference value of $0.033 \mu \mathrm{g} \mathrm{g}^{-1}$ $\mathrm{ww}$ for $\mathrm{Hg}$ in food for all wildlife that consumes aquatic organisms. The reference value for $\sum \mathrm{DDT}$ is set at 14 $\mathrm{ng} \mathrm{g}^{-1} \mathrm{ww}$ in food. In the present work, we found that $41 \%$ of bivalves, $76 \%$ of crustaceans, $76 \%$ of fish, and $96 \%$ of sharks and rays exceeded the limit for $\mathrm{Hg}$, while $15 \%$ of bivalves, $26 \%$ of crustaceans, and $40 \%$ of fish exceeded the limit for $\sum$ DDTs. These results show the risks that wildlife face in aquatic ecosystems in Mexico.

The potential risk to human health from the consumption of PBTS through food consumption of aquatic origin is a global concern. Various diseases have been associated with exposure to low concentrations of contaminants, such as cancer, neurotoxicity, cardiovascular diseases, endocrine disruption, and immunological disorders (Dórea, 2008). As a result, various national and international agencies have established maximum permissible limits, seeking to protect consumers from the adverse effects of consuming toxic residues. The USEPA, for example, establishes maximum permissible limits of 1 and $0.1 \mu \mathrm{g}$ $\mathrm{g}^{-1} \mathrm{ww}$ for $\mathrm{Cd}$ and $\mathrm{Hg}$ in fish, respectively. The European Community (EC) establishes maximum permissible of $1,0.5$, and $0.5 \mu \mathrm{g} \mathrm{g}^{-1}$ ww for $\mathrm{Hg}, \mathrm{Cd}$, and $\mathrm{Pb}$ in fish muscle, respectively. In contrast, the INECC (Mexico) establishes maximum permissible limits of 1 , 0.5 , and $1 \mu \mathrm{g} \mathrm{g}^{-1} \mathrm{ww}$ for $\mathrm{Hg}, \mathrm{Cd}$, and $\mathrm{Pb}$, respectively.

In the present review, we found that close to $90 \%$ of the cases of mollusks, crustaceans, fish, sharks, and rays did not involve average concentrations exceeding the limit of $0.5 \mu \mathrm{g} \mathrm{g}^{-1} \mathrm{ww}$ for $\mathrm{Hg}$. In the case of fish, only Caranx caninus collected in Topolobampo Bay, Sinaloa, in 2004, exceeded this limit, with a $\mathrm{Hg}$ concentration in the muscle of $1 \mu \mathrm{g} \mathrm{g}^{-1}$ ww. However, about $35 \%$ of shark cases exceeded this value, even exceeding the reference value of $1 \mu \mathrm{g} \mathrm{g}^{-1}$ ww for $\mathrm{Hg}$. An extreme case involved a specimen of $S$. zygaena collected in the Gulf of California, with $\mathrm{Hg}$ levels exceeding $8 \mu \mathrm{g} \mathrm{g}^{-1} \mathrm{ww}$. In contrast, about $90 \%$ of fish, sharks, and crustaceans showed Cd levels in muscle lower than $0.5 \mu \mathrm{g} \mathrm{g}^{-1} \mathrm{ww}$. Regarding mollusks, about $45 \%$ of the organisms reviewed exceeded the limit of $0.5 \mu \mathrm{g} \mathrm{g}^{-1} \mathrm{ww}$ for $\mathrm{Cd}$, while $18 \%$ exceeded $1 \mu \mathrm{g} \mathrm{g}^{-1} \mathrm{ww}$, and close to $20 \%$ exceeded the limit of $1 \mu \mathrm{g} \mathrm{g}^{-1} \mathrm{Ww}$ for $\mathrm{Pb}$. For $\sum \mathrm{DDT}$, the EC establishes a maximum permissible limit of $1000 \mathrm{ng} \mathrm{g}^{-1}$, while the Codex Alimentarius Guidelines of the Food and Agriculture Organization of the United Nations (USFDA) and the World Health Organization (WHO) establishes a maximum permissible limit of 5,000 $\mathrm{ng} \mathrm{g}^{-1}$. All the cases reviewed in this work were below the limit of $1,000 \mathrm{ng} \mathrm{g}^{-1}$ for $\sum$ DDTs.

The potential risk of fish consumption to human health can be evaluated considering a risk ratio (HQ) defined by the equation HQ = E / RfD (Newman \& Unger, 2002); where RfD is the reference dose (in $\mu \mathrm{g}$ $\mathrm{kg}^{-1} \mathrm{~kg}^{-1}$ of weight $\mathrm{d}^{-1}$ ), and $\mathrm{E}$ is the level of exposure or consumption of the contaminant, which is calculated using the equation $\mathrm{E}=\mathrm{C} \times \mathrm{I} / \mathrm{W}$, where $\mathrm{C}$ is the concentration of the contaminant (in $\mu \mathrm{g} \mathrm{g}^{-1} \mathrm{ww}$ ), I is the per capita intake rate (in $\mathrm{g} \mathrm{d}^{-1}$ ), and $\mathrm{W}$ is the average weight (in $\mathrm{kg}$ ). Considering an RfD of 0.3, 0.5, 0.5, 1, and 3.5 for $\sum \mathrm{HCHs}, \sum \mathrm{DDTs}, \mathrm{Hg}, \mathrm{Cd}$, and $\mathrm{Pb}$, respectively, I of $30 \mathrm{~g} \mathrm{~d}^{-1}$ for Mexicans consumers, and $\mathrm{W}$ of $70 \mathrm{~kg}$, only in about $5 \%$ of cases does the value of HQ exceeds 1 for all contaminants. However, in some communities with high fish consumption rates, with an RfD of 200-400 g/d, the risk increases considerably, especially in infants and pregnant women (Zamora-Arellano et al., 2017; Astorga-Rodríguez et al., 2018).

\section{CONCLUSIONS}

This review synthesizes a large amount of information on the biomonitoring of the main PBTS in aquatic ecosystems in Mexico, and this information can serve as a basis for future research. In general, the levels of pollution found in Mexico's aquatic ecosystems are similar to those found in other regions of the world. However, given Mexico's enormous ecological wealth, especially its aquatic ecosystems, there is insufficient information on environmental pollutants. Moreover, there is a great disparity in the amount of information available between the country's different areas. Just the Gulf of California concentrates about $80 \%$ of the studies reviewed here, which shows the need to increase research efforts in the country's currently neglected areas such as the Central Pacific or the 
Mexican Caribbean, whose participation in this work did not exceed $5 \%$.

\section{REFERENCES}

Aguilar, C.A., Montalvo, C., Rodríguez, L., Cerón, J.G. \& Cerón, R.M. 2012. American oyster (Crassostrea virginica) and sediments as a coastal zone pollution monitor by heavy metals. International Journal of Environmental Science and Technology, 9: 579-586.

Aguilar-Betancourt, C.M., González-Sansón, G., Kidd, K.A., Munkittrick, K.R., Curry, R.A., KosonoyAceves, D., et al. 2016. Fishes as indicators of untreated sewage contamination in a Mexican coastal lagoon. Marine Pollution Bulletin, 113(1): 100-109.

Aguilar-Ucán, C.A., Montalvo-Romero, C., CerónBretón, J.G. \& Anguebes-Fransesch, F. 2014. Levels of heavy metals in marine species: Oyster (Crassostrea virginica), crab (Callinectes sapidus) and shrimp (Litopenaeus setiferus) of Ciudad del Carmen, Campeche, México. Revista Latinoamericana de Recursos Naturales, 10(1): 9-17.

Akcali, I. \& Kucuksezgin, F. 2011. A biomonitoring study: heavy metals in macroalgae from eastern Aegean coastal areas. Marine Pollution Bulletin, 62: 637-645.

Álvarez-Moya, C. \& Reynoso-Silva, M. 2015. Use of comet assay in human lymphocytes as a molecular biomarker for simultaneous monitoring of genetic damage and genotoxicity in residents who lived nearby the Santiago River, Mexico, in 2012. Global Journal of Biotechnology and Biomaterial Science, 1(1): 4-8.

Aranguré-Zúniga, F.J., Zambrano, R.M., González, L. \& Robledo, M. 2011. Evaluation of contamination levels by organochlorine pesticides residues on sediment and fishes from Aguamilpa reservoir, Nayarit, México. Waxapa, 3(4): 20-35.

Astorga-Rodríguez, J.E., Martínez-Rodríguez, I.E., García-de la Parra, L.M., Betancourt-Lozano, M., Vanegas-Pérez, R.C., Ponce de León-Hill, C.A. \& Ruelas-Inzunza, J. 2018. Lead and cadmium levels in mussels and fishes from three coastal ecosystems of NW Mexico and its potential risk due to fish and seafood consumption. Toxicology and Environmental Health Sciences, 10(3): 203-211.

Astudillo, L.R., Yen, I.C. \& Bekele, I. 2005. Heavy metals in sediments, mussels and oysters from Trinidad and Venezuela. Revista de Biología Tropical, 53(1): 41-53.

Avelar, M., Bonilla-Heredia, B., Merino-Ibarra, M., Herrera-Silveira, J.A., Ramírez, J., Rosas, H., et al. 2013. Iron, cadmium, and chromium in seagrass (Thalassia testudinum) from a coastal nature reserve in karstic Yucatán. Environmental Monitoring and Assessment, 185: 7591-7603.
Barrera-García, A., O'Hara, T., Galván-Magaña, F., Méndez-Rodríguez, L.C., Castellini, J.M. \& ZentenoSavín, T. 2012. Oxidative stress indicators and trace elements in the blue shark (Prionace glauca) off the east coast of the Mexican Pacific Ocean. Comparative Biochemistry and Physiology Part C: Toxicology \& Pharmacology, 156: 59-66.

Barrera-García, A., O'Hara, T., Galván-Magaña, F., Méndez-Rodríguez, L.C., Castellini, J.M. \& ZentenoSavín, T. 2013. Trace elements and oxidative stress indicators in the liver and kidney of the blue shark (Prionace glauca). Comparative Biochemistry and Physiology Part A: Molecular \& Integrative Physiology, 165(4): 483-490.

Benítez, J.A., Vidal, J., Brichieri-Colombi, T. \& DelgadoEstrella, A. 2012. Monitoring ecosystem health of the Terminos Lagoon region using heavy metals as environmental indicators. Environmental Impact Assessment Review, 162: 349-358.

Bergés-Tiznado, M.E., Márquez-Farías, F., LaraMendoza, R.E., Torres-Rojas, Y.E., Galván-Magaña, F., Bojórquez-Leyva, H. \& Páez-Osuna, F. 2015 Mercury and selenium in muscle and target organs of scalloped hammerhead sharks Sphyrna lewini of the SE Gulf of California: dietary intake, molar ratios, loads, and human health risks. Archives of Environmental Contamination and Toxicology 69: 440-452.

Buenfil-Rojas, A.M., Álvarez-Legorreta, T. \& CedeñoVázquez, J.R. 2015. Metals and metallothioneins in morelet's crocodile (Crocodylus moreletii) from a Transboundary River Between Mexico and Belize. Archives of Environmental Contamination and Toxicology, 68: 265-273.

Cadena-Cárdenas, L., Méndez-Rodríguez, L., ZentenoSavín, T., García-Hernández, J. \& Acosta-Vargas, B. 2009. Heavy metal levels in marine mollusks from areas with, or without, mining activities along the Gulf of California, Mexico. Archives of Environmental Contamination and Toxicology, 57: 96-102.

Calderón-Villagómez, H.E., González, E.R. \& Durán, C. 2001. Plaguicidas organoclorados en sedimentos y organismos acuáticos del Lago de Catemaco, Veracruz, México. Revista Internacional de Contaminación Ambiental, 17(1): 23-30.

Canadian Council of Ministers of the Environment (CCME). 1998. Protocol for derivation of Canadian tissue residue guidelines for the protection of wildlife consumers of aquatic biota. Winnipeg, Manitoba.

Cantú-Medellín, N., Olguín-Monroy, N.O., MéndezRodríguez, L.C. \& Zenteno-Savín, T. 2009. Antioxidant Enzymes and Heavy metal levels in tissues of the black chocolate clam megapitaria squalida in Bahía 
de La Paz, Mexico. Archives Environmental Contamination and Toxicology, 56: 60-66.

Carvalho, F.P., Jean-Pierre, V., Cattini, C., Rendón, J. \& Mota, J. 2009. Ecological risk assessment of PCBs and other organic contaminant residues in Laguna de Terminos, Mexico. Ecotoxicology, 18: 403-416.

Carvalho, F.P., González-Farias, F., Villeneuve, J.P., Cattini, C., Hernández-Garza, M., Mee, L.D. \& Fowler, S.W. 2002. Distribution, fate and effects of pesticide residues in tropical coastal lagoons of northwestern Mexico. Environmental Technology, 23(11): 1257-1270.

Castañeda-Chávez, M.R., Lango-Reynoso F. \& LanderosSánchez, C. 2011. DDT in Crassostrea virginica (Gmelin, 1791) of coastal lagoons in the Gulf of Mexico. Journal of Agricultural Science, 3(1): 183193.

Castañeda-Chávez, M.R., Navarrete-Rodríguez, G., Lango-Reynoso, F., Galaviz-Villa, I. \& LanderosSánchez, C. 2014. Heavy metals in oysters, shrimps and crabs from lagoon systems in the southern Gulf of México. Journal of Agricultural Science 6(3): 108117.

Ceyca, J.P., Castillo-Guerrero, J.A., García-Hernández, J., Fernández, G. \& Betancourt-Lozano, M. 2016. Local and interannual variations in mercury and cadmium in eggs of eight seabird species of the Sinaloa coast, Mexico. Environmental Toxicology and Chemistry, 35(9): 2330-2338.

Comisión Nacional del Agua (CONAGUA). 2017. Estadísticas del agua en México. Secretaría de Medio Ambiente y Recursos Naturales. Comisión Nacional del Agua, Ciudad de México.

Cortez-Gómez, A.A., Fuentes-Mascorro, G. \& Romero, D. 2014. Metals and metalloids in whole blood and tissues of Olive Ridley turtles (Lepidochelys olivacea) from La Escobilla Beach (Oaxaca, Mexico). Marine Pollution Bulletin, 89: 367-375.

Delgado-Álvarez, C.G., Ruelas-Inzunza, J., Osuna-López, J.I., Voltolina, D. \& Frías-Espericueta, M.G. 2015a. Mercury content and their risk assessment in farmed shrimp Litopenaeus vannamei from NW Mexico. Chemosphere, 119: 1015-1020.

Delgado-Álvarez, C.G., Ruelas-Inzunza, J., Osuna-López, J.I., Voltolina, D. \& Frías-Espericueta, M.G. 2015b. Total mercury content in cultured oysters from NW Mexico: health risk assessment. Bulletin of Environmental Contamination and Toxicology, 94: 209-213.

Díaz-González, G., Botello, A.V. \& Ponce-Vélez, G. 2005. Plaguicidas organoclorados en pastos y peces de los sistemas Candelaria-Panlau y Palizada del Este, Laguna de Términos, Campeche, México. In: Botello, A.V., Rendón-von-Osten, J., Gold-Bouchot, G. \& Agraz-Hernández, C. (Eds.). Golfo de México.
Contaminación e impacto ambiental: diagnóstico y tendencias. Universidad Autónoma de Campeche, Universidad Nacional Autónoma de México, Instituto Nacional de Ecología, Ciudad de México, pp. 207-224.

Díaz-López, C., Carrión-Jiménez, J.M. \& GonzálezBucio, J.L. 2006. Estudio de la contaminación por Hg, $\mathrm{Pb}, \mathrm{Cd}$ y Zn en la Bahía de Chetumal, Quintana Roo, México. Revista Sociedad Química del Perú, 72: 9-31.

Dórea, J.G. 2008. Persistent, bioaccumulative and toxic substances in fish: human health considerations. Science of the Total Environment, 400: 93-114.

Elliott, J.E., Kirk, D.A., Elliott, K.H., Dorzinsky, J., Lee, S., Ruelas, E., Cheng, K.M.T., Scheuhammer, T. \& Shaw, P. 2015. Mercury in forage fish from Mexico and Central America: implications for fish-eating birds. Archives of Environmental Contamination and Toxicology, 69(4): 375-389.

Escobar-Sánchez, O., Galván-Magaña, F. \& RosílesMartínez, R. 2011. Biomagnification of mercury and selenium in blue shark Prionace glauca from the Pacific Ocean off Mexico. Biological Trace Element Research, 144: 550-559.

Escobar-Sánchez, O., Ruelas-Inzunza, J., MorenoSánchez, X.G., Romo-Piñera, A.K. \& FríasEspericueta, M.G. 2016. Mercury concentrations in pacific angel sharks (Squatina californica) and prey fishes from Southern Gulf of California, Mexico. Bulletin of Environmental Contamination and Toxicology, 96(1): 15-19.

Escobar-Sánchez, O., Ruelas-Inzunza, J., Patrón-Gómez, J.C. \& Corro-Espinosa, D. 2014. Mercury levels in myliobatid stingrays (Batoidea) from the Gulf of California: tissue distribution and health risk assessment. Environmental Monitoring and Assessment, 186: 1931-1937.

Escobedo-Fregoso, C., Mendez-Rodriguez, L.C., Monsalvo-Spencer, P., Llera-Herrera, R.A., ZentenoSavin, T. \& Acosta-Vargas, B. 2010. Assessment of metallothioneins in tissues of the clam megapitaria squalida as biomarkers for environmental cadmium pollution from areas enriched in phosphorite. Archives Environmental Contamination Toxicology, 59: 255263.

Espinosa-Reyes, G., González-Mille, D.J., IlizaliturriHernández, C.A., Díaz-Barriga, F.M. \& MejíaSaavedra, J. 2012. Exposure assessment to persistent organic pollutants in wildlife: the case study of Coatzacoalcos, Veracruz, Mexico, organic pollutants ten years after the Stockholm convention. In: Puzyn, T. \& Mostrag-Szlichtyng, A. (Eds.). Environmental and Analytical Update. InTech Open, London.

Faria, M., López, M.A., Díez, S. \& Barata, C. 2010. Are native naiads more tolerant to pollution than exotic freshwater bivalve species? An hypothesis tested using 
physiological responses of three species transplanted to mercury contaminated sites in the Ebro River (NE, Spain). Chemosphere, 81: 1218-1226.

Fernández-Bringas, L.M., Ponce-Vélez, G., Calva, L.G., Salgado-Ugarte, I.H., Botello A.V. \& Díaz, G. 2008. Organochlorine pesticides in lacustrine sediments and tilapias of Metztitlan, Hidalgo, México. Revista de Biología Tropical, 56(3): 1381-1390.

Food and Agriculture Organization (FAO). 2018. El estado mundial de la pesca y la acuicultura. Cumplir los objetivos de desarrollo sostenible. FAO, Rome.

Frías-Espericueta, M.G., Osuna-López, J.I., EstradaToledo, F.J., López-López, G. \& Izaguirre-Fierro, G. 2005a. Heavy metals in the edible muscle of shrimp from coastal lagoons located in northwest Mexico. Bulletin of Environmental Contamination and Toxicology, 74: 1098-1104.

Frías-Espericueta, M.G., Osuna-López, J.I., Flores-Reyes, S., López-López, G. \& Izaguirre-Fierro, G. 2005b. Heavy metals in the oyster Crassostrea corteziensis from Urias Lagoon, Mazatlán, Mexico, associated with different anthropogenic discharges. Bulletin of Environmental Contamination and Toxicology, 74: 996-1002.

Frías-Espericueta, M.G., Osuna-López, J.I., Voltolina, D., López-López, G., Izaguirre-Fierro, G. \& Muy-Rangel, M.D. 2008. The metal content of bivalve mollusks of a coastal lagoon of NW Mexico. Bulletin of Environmental Contamination and Toxicology, 80: 90-92.

Frías-Espericueta, M.G., Osuna-López, J.I., Ruiz-Telles, A., Quintero-Álvarez, J.M., López-López, G., Izaguirre-Fierro, G. \& Voltolina, D. 2006. Heavy metals in the tissues of the sea turtle Lepidochelys olivacea from a nesting site of the northwest coast of Mexico. Bulletin of Environmental Contamination and Toxicology, 77: 179-185.

Frías-Espericueta, M.G., Quintero-Álvarez, J.M., OsunaLópez, J.I., Sánchez-Gaxiola, C.M., López-López, G., Izaguirre-Fierro, G. \& Voltolina, D. 2010. Metal contents of four commercial fish species of NW Mexico. Bulletin of Environmental Contamination and Toxicology, 85: 334-338.

Frías-Espericueta, M.G., Vargas-Jiménez, A., RuelasInzunza, J., Osuna-López, J.I., Aguilar-Juárez, M., Bautista-Covarrubias, J.C. \& Voltolina, D. 2016. Total mercury in Mugil spp. and Eugerres axillaris of a subtropical lagoon of NW Mexico. Bulletin of Environmental Contamination and Toxicology, 97: 211-215.

Frías-Espericueta, M.G., Osuna-López, J.I., JiménezVega, M.A., Castillo-Bueso, D., Muy-Rangel, M.D., Rubio-Carrasco, W., et al. 2011. Cadmium, copper, lead, and zinc in Mugil cephalus from seven coastal lagoons of NW Mexico. Environmental Monitoring and Assessment, 182: 133-139.

Frías-Espericueta, M.G., Osuna-López, J.I., Voltolina, D., Beltrán-Velarde, M.A., Izaguirre-Fierro, G., LópezLópez. G., et al. 2009. The contents of $\mathrm{Cd}, \mathrm{Cu}, \mathrm{Pb}$ and $\mathrm{Zn}$ of the white shrimp Litopenaeus vannamei (Boone, 1931) of six coastal lagoons of Sinaloa, NW Mexico. Revista de Biología Marina y Oceanografía, 44(1): 197-201.

Gallo-Reynoso, J.P., Malek, T.B., García-Hernández, J., Vázquez-Moreno, L. \& Segura-García, I. 2014. Concentrations of DDE in blubber biopsies of freeranging long-beaked common dolphin (Delphinus capensis) in the Gulf of California. Bulletin of Environmental Contamination and Toxicology, 94(1): 6-11.

García-Besné, G., Valdespino, C. \& Rendón-von Osten, J. 2014. Comparison of organochlorine pesticides and PCB residues among hawksbill (Eretmochelys imbricata) and green (Chelonia mydas) turtles in the Yucatan Peninsula and their maternal transfer. Marine Pollution Bulletin, 91(1): 139-148.

García-Hernández, J., Glenn, E.P. \& Flessa, K. 2013. Identification of chemicals of potential concern (COPECs) in anthropogenic wetlands of the Colorado River delta. Ecological Engineering, 59: 52-60.

García-Hernández, J., García-Rico L., Jara-Marini M.E., Barraza-Guardado, R. \& Hudson, A. 2005. Concentrations of heavy metals in sediment and organisms during a harmful algal bloom (HAB) at Kun Kaak Bay, Sonora, Mexico. Marine Pollution Bulletin, 50: 733739.

García-Hernández, J., Cadena-Cárdenas, L., Betancourt, M., García-De-La-Parra, L.M., García-Rico, L. \& Márquez-Farías, F. 2007. Total mercury content found in edible tissues of top predator fish from the Gulf of California, Mexico. Toxicology Environmental Chemistry, 89(3): 507-522.

García-Hernández, J., Espinosa-Romero, M.J., CisnerosMata, M.A., Leyva-García, G., Aguilera-Márquez, D. \& Torre, J. 2015. Concentración de mercurio y plaguicidas organoclorados (poc) en tejido comestible de jaiba café Callinectes bellicosus de las costas de Sonora y Sinaloa, México. Ciencia Pesquera, 23: 6579.

García-Hernández, J., King, K.A., Velasco, A.L., Shumilin, E., Mora, M.A. \& Edward, P. 2001. Selenium, selected inorganic elements, and organochlorine pesticides in bottom material and biota from the Colorado River Delta. Journal of Arid Environments, 49: 65-89.

García-Hernández, J., Sapozhnikova, Y.V., Schlenk, D., Mason, A.Z., Hinojosa-Huerta, O., Rivera-Díaz, J.J., et al. 2006. Concentration of contaminants in breeding bird eggs from the Colorado River Delta, Mexico. 
Environmental Toxicology and Chemistry, 25(6): 1640-1647.

García-Rico, L. \& Ramos-Ruiz, R.E. 2001. Determination of total metals in cultivated Oysters (Crassostrea gigas) from the Northwest Coast of Mexico by Microwave Digestion and Atomic Absorption Spectrometry. Journal of AOAC International, 84(6): 19091913.

García-Rico, L., Tejeda-Valenzuela, L. \& BurgosHernández, A. 2010. Seasonal variations in the concentrations of metals in Crassostrea corteziensis from Sonora, México. Bulletin of Environmental Contamination and Toxicology, 85: 209-213.

García-Ríos, V.Y. \& Gold-Bouchot, G. 2002. Especiación de metales pesados en sedimentos de la Bahía de Chetumal, Quintana Roo, y la acumulación en el tejido muscular de bagres (Ariopsis assimilis). In: RosadoMay, F.J., Romero-Mayo, R. \& De Jesús Navarrete, A. (Eds.). Contribuciones de la ciencia al manejo costero integrado de la Bahía de Chetumal y su área de influencia. Universidad de Quintana Roo, Quintana Roo, pp. 143-148.

Gardner, S.C., Dawn, M., Wesselman, R. \& Juárez, J.A. 2003. Organochlorine contaminants in sea turtles from the Eastern Pacific. Marine Pollution Bulletin, 46: 1082-1089.

Gardner, S.C., Fitzgerald, S.L., Acosta, V.B. \& Méndez, L. 2006. Heavy metal accumulation in four species of sea turtles from the Baja California Peninsula, Mexico. BioMetals, 19: 91-99.

Gold-Bouchot, G., Zapata-Pérez, O., Ceja-Moreno, V., Rodríguez-Fuentes, G., Simá-Alvarez, R., AguirreMacedo, M.L. \& Vidal-Martínez, V.M. 2007. Biological effects of environmental pollutants in American oyster, Crassostrea virginica: a field study in Laguna de Terminos, Mexico. International Journal of Environment and Health, 1(2): 171-184.

Gómez-Meda, B.C., Zúñiga-González, G.M., SánchezOrozco, L.V., Zamora-Perez, A.L., Rojas-Ramírez, J.P., Rocha-Muñoz, A.D., et al. 2017. Buccal micronucleus cytome assay of populations under chronic heavy metal and other metal exposure along the Santiago River, Mexico. Environmental Monitoring and Assessment, 189(10): 522. doi: 10.1007/s 10661-017-6237-3

Gonzalez-Jauregui, M., Valdespino, C., Salame-Méndez, A., Aguirre-Leon, G. \& Rendón-von Osten. J. 2012. Persistent organic contaminants and steroid hormones levels in morelet's crocodiles from the Southern Gulf of Mexico. Archives of Environmental Contamination and Toxicology, 62: 445-454.

González-Mille, D.J., Ilizaliturri-Hernández, C.A., Espinosa-Reyes, G., Costilla-Salazar, R., DíazBarriga, F., Ize-Lema, I. \& Mejía-Saavedra, J. 2010. Exposure to persistent organic pollutants (POPs) and
DNA damage as an indicator of environmental stress in fish of different feeding habits of Coatzacoalcos, Veracruz, Mexico. Ecotoxicology, 19(7): 1238-1248.

Granados-Galván, I.A., Rodríguez-Meza, D.G., LunaGonzález, A. \& González-Ocampo, H.A. 2015. Human health risk assessment of pesticide residues in snappers (Lutjanus) fish from the Navachiste Lagoon complex, Mexico. Marine Pollution Bulletin, 97(1-2): 178-187.

Green-Ruiz, C., Ruelas-Inzunza, J. \& Páez-Osuna, F. 2005. Mercury in Surface sediments and benthic organisms from Guaymas Bay, east coast of the Gulf of California. Environmental Geochemistry and Health, 27: 321-329.

Guentzel, J.L., Portilla, E., Keith, K.M. \& Keith, E.O. 2007. Mercury transport and bioaccumulation in riverbank communities of the Alvarado Lagoon System, Veracruz State, Mexico. Science of the Total Environment, 388: 316-324.

Guzmán-García, X., Botello, A.V., Martínez-Tabche, L. \& González-Márquez, H. 2009. Effects of heavy metals on the oyster (Crassostrea virginica) at Mandinga Lagoon, Veracruz, Mexico. Revista de Biología Tropical, 57(4): 955-962.

Hicks, E.A. 1976. Variación estaciona1 en la concentración de elementos metálicos en ostiones de la laguna de Términos, Campeche, México. Tesis profesional, Facultad de Química, Universidad Nacional Autónoma de México, Ciudad de México, 50 pp.

Higueras, P., Oyarzun, R., Lillo, J., Sánchez-Hernández, J.C., Molina, J. A., Esbrí, J.M. \& Lorenzo, S. 2006. The Almadén district (Spain): anatomy of one of the world's largest $\mathrm{Hg}$-contaminated sites. Science of the Total Environment, 356: 112-124.

Hinojosa-Garro, D., Burgos, A.M. \& Rendón-von Osten, J. 2016. Organochlorine pesticides (ocps) in sediment and fish of two tropical water bodies under different land use. Bulletin of Environmental Contamination and Toxicology, 97(1): 105-111.

Hurtado-Banda, R., Gómez-Álvarez, A., Márquez-Farías, J.F., Córdoba-Figueroa, M., Navarro-García, G. \& Medina-Juárez, L.A. 2012. Total mercury in liver and muscle tissue of two coastal sharks from the Northwest of Mexico. Bulletin of Environmental Contamination and Toxicology, 88: 971-975.

Instituto Nacional de Ecología y Cambio Climático (INECC). 2017. Contaminación y salud ambiental. Integración del inventario nacional de emisiones y liberaciones de mercurio, 2015. Coordinación General de Contaminación y Salud Ambiental del INECC. Secretaría de Medio Ambiente y Recursos Naturales, Ciudad de México. 
Jara-Marini, M.E., Soto-Jiménez, M.F. \& Páez-Osuna, F. 2008. Trace metals accumulation patterns in a mangrove lagoon ecosystem, Mazatlan Harbor, southeast Gulf of California. Journal of Environmental Science and Health, Part A, 43(9): 995-1005.

Jara-Marini, M.E., Soto-Jiménez, M.F. \& Páez-Osuna, F. 2012. Mercury transfer in a subtropical coastal lagoon food web (SE Gulf of California) under two contrasting climatic conditions. Environmental Toxicology, 27(9): 526-536.

Jara-Marini, M.E., Tapia-Alcaraz, J.N., Dumer-Gutiérrez, J.A., García-Rico, L., García-Hernández, J. \& PáezOsuna, F. 2013. Comparative bioaccumulation of trace metals using six filter feeder organisms in a coastal lagoon ecosystem (of the central-east Gulf of California). Environmental Monitoring and Assessment, 185: 1071-1085.

Kampalath, R., Gardner, S., Méndez-Rodríguez, L. \& Jay, J. 2006. Total and methylmercury in three species of sea turtles of Baja California Sur. Marine Pollution Bulletin, 52: 1784-1832.

Labrada-Martagón, V., Tenorio, P.A., MéndezRodríguez, L.C. \& Zenteno-Savín, T. 2011. Oxidative stress indicators and chemical contaminants in East Pacific Green turtles (Chelonia mydas) inhabiting two foraging coastal lagoons in the Baja California Peninsula. Comparative Biochemistry and Physiology Part C: Toxicology \& Pharmacology, 154: 65-75.

Lango-Reynoso, F., Landeros-Sánchez, C. \& CastañedaChávez, R.M. 2010. Bioaccumulation of cadmium $(\mathrm{Cd})$, lead $(\mathrm{Pb})$ and arsenic (As) in Crassostrea virginica (Gmelin, 1791), from Tamiahua Lagoon System, Veracruz, México. Revista Internacional de Contaminación Ambiental, 26(3): 201-210.

Lango-Reynoso, F., Castañeda-Chávez, M.R., LanderosSánchez, C., Galavíz-Villa, I., Navarrete-Rodríguez, G. \& Soto-Estrada, A. 2013. Cd, Cu, $\mathrm{Hg}$ and Pb, and organochlorine pesticides in commercially important benthic organism's coastal lagoons SW Gulf of Mexico. Agricultural Science, 1(1): 63-79.

Lerma, M., Castillo-Guerrero, J.A., Ruelas-Inzunza, J. \& Fernández, G. 2016. Lead, cadmium and mercury in the blood of the blue-footed booby (Sula nebouxii) from the coast of Sinaloa, Gulf of California, Mexico. Marine Pollution Bulletin, 110(1): 293-298.

Ley-Quiñónez, C., Zavala-Norzagaray, A.A., EspinosaCarreón, T.L., Peckham, H., Marquez-Herrera, C., Campos-Villegas, L. \& Aguirre, A.A. 2011. Baseline heavy metals and metalloid values in blood of loggerhead turtles (Caretta caretta) from Baja California Sur, Mexico. Marine Pollution Bulletin, 62: 1979-1983.

Ley-Quiñónez, C.P., Zavala-Norzagaray, A.A., RéndonMaldonado, J.G., Espinosa-Carreón, T.L., Canizales-
Román, A., Escobedo-Urías, D.C., et al. 2013. Selected heavy metals and selenium in the blood of black sea turtle (Chelonia mydas agasiizzi) from Sonora, Mexico. Bulletin of Environmental Contamination and Toxicology, 91: 645-651.

Li, J., Zhou, Q., Yuan, G., He, X. \& Xie, P. 2015. Mercury bioaccumulation in the food web of Three Gorges Reservoir (China): tempo-spatial patterns and effect of reservoir management. Science of the Total Environment, 527: 203-210.

Lino, A.S., Galvão, P.M.A. Longo, R.T.L., AzevedoSilva, C.E., Dorneles, P.R., Torres, J.P.M. \& Malm, O. 2016. Metal bioaccumulation in consumed marine bivalves in Southeast Brazilian coast. Journal of Trace Elements in Medicine and Biology, 34: 50-55.

Liu, J., Cao, L. \& Dou, S. 2017. Bioaccumulation of heavy metals and health risk assessment in three benthic bivalves along the coast of Laizhou Bay, China. Marine Pollution Bulletin, 117(1): 98-110.

Luna-Porres, M.Y., Rodríguez-Villa, M.A., HerreraPeraza, E.F., Renteria-Villalobos, M. \& MonteroCabrera, M.E. 2014. Potential human health risk by metal (loid)s, 234,238U and 210Po due to consumption of fish from the "Luis L. Leon" Reservoir (northern México). International Journal of Environmental Research and Public Health, 11: 66126638.

Macías-Zamora, J.V., Sánchez-Osorio, J.L., RíosMendoza, L.M., Ramírez-Álvarez. N., Huerta-Díaz, M.A. \& López-Sánchez, D. 2008. Trace metals in sediments and Zostera marina of San Ignacio and Ojo de Liebre lagoons in the Central Pacific coast of Baja California, México. Archives of Environmental Contamination and Toxicology, 55: 218-228.

Maldonado-Enríquez, E.J., López-Noverola, U., SalinasHernández, R.M., González-Cortés, N., Cuenca-Soria, C.A., Jiménez-Vera, R. \& Hernández-Juárez, J.L. 2015. Contenido de metales pesados en músculo de pez diablo Pterygoplichthys pardalis. Revista Iberoamericana de Ciencias, 2(1): 67-73.

Martínez-Villa, G., Betancourt-Lozano, M., AguilarZárate, G., Ruelas-Inzunza, J., Anislado-Tolentino, V., Cerdenares-Ladrón de Guevara, G., Ramos-Carrillo, S. \& González-Medina, G. 2014. Contenido de plaguicidas organoclorados en varios peces depredadores de la costa de Oaxaca y evaluación del riesgo de exposición por consumo en la salud humana. In: Botello, A.V., Páez-Osuna, F., Mendez-Rodríguez, L., Betancourt-Lozano, M., Álvarez-Borrego, S. \& LaraLara, R. (Eds.). Pacific Mexicano. Contaminación e impacto ambiental: diagnóstico y tendencias. UAC, UNAM-ICMYL， CIAD-MAZATLÁN， CIBNOR, CICESE, pp. 169-208.

Maz-Courrau, A., López-Vera, C., Galván-Magaña, F., Escobar-Sánchez, O., Rosíles-Martínez, R. \& 
Sanjuán-Muñoz, A. 2012. Bioaccumulation and biomagnification of total mercury in four exploited shark species in the Baja California Peninsula, Mexico. Bulletin of Environmental Contamination and Toxicology, 88: 129-134.

McCulligh, C. 2014. Contaminar para competir. Contaminación industrial del Río Santiago en Jalisco. Carta Económica Regional, 26(113): 114-137.

Mellink, E., Riojas-López, M.E. \& Luévano-Esparza, J. 2009. Organochlorine content and shell thickness in brown booby (Sula leucogaster) eggs in the Gulf of California and the southern Pacific coast of Mexico. Environmental Pollution, 157: 2184-2188.

Méndez, L., Palacios, E., Acosta, B., Monsalvo-Spencer, P. \& Álvarez-Castañeda, T. 2006. Heavy metals in the clam Megapitaria squalida collected from wild and phosphorite mine-impacted sites in Baja California, Mexico. Considerations for human health effects. Biological Trace Element Research, 110: 275-287.

Méndez, L., Salas-Flores L.M., Arreola-Lizarraga A., Alvarez-Castañeda S.T. \& Acosta, B. 2002. Heavy metals in clams from Guaymas Bay, Mexico. Mexico. Bulletin of Environmental Contamination and Toxicology, 68: 217-223.

Mendoza-Carranza, M., Sepúlveda-Lozada, A., DíasFerreira, C. \& Geissen, V. 2016. Distribution and bioconcentration of heavy metals in a tropical aquatic food web: a case study of a tropical estuarine lagoon in SE Mexico. Environmental Pollution, 210: 155-165.

Morachis-Valdez, G., Dublán-García, O., LópezMartínez, L.X., Galar-Martínez, M., Saucedo-Vence, K. \& Gómez-Oliván, L.M. 2015. Chronic exposure to pollutants in Madín Reservoir (Mexico) alters oxidative stress status and flesh quality in the common carp Cyprinus carpio. Environmental Science and Pollution Research, 22: 9159-9172.

Morales-Hernández, F., Soto-Jiménez M.F. \& PáezOsuna, F. 2004. Heavy metals in sediments and lobster (Panulirus gracilis) from the discharge area of the submarine sewage outfall in Mazatlán Bay (SE Gulf of California). Archives of Environmental Contamination and Toxicology, 46: 485-491.

Neufeld, D.S.G. 2010. Mercury accumulation in caged Corbicula: rate of uptake and seasonal variation. Environmental Monitoring and Assessment, 168: 385396.

Newman, M.C. \& Unger, M.A. 2002. Fundamentals of ecotoxicology. CRC Press, Florida.

Niño-Torres, C.A., Gardner, S.C. Zenteno-Savín, T. \& Ylitalo, G.M. 2009. Organochlorine pesticides and polychlorinated biphenyls in California sea lions (Zalophus californianus californianus) from the Gulf of California, México. Archives of Environmental Contamination and Toxicology, 56(2): 350-359.
Núñez-Noriega, G. 2005. Concentration of essential and non-essential metals in two shark species commonly caught in Mexican (Gulf of Mexico) coastline. In: Botello, A.V., Rendón-von Osten, J. \& AgrazHernández, C. (Eds.). Golfo de México. Contaminación e impacto ambiental: diagnóstico y tendencias. Universidad Autónoma de Campeche, Universidad Autónoma de México, Instituto Nacional de Ecología, Ciudad de México, pp. 451-474.

Orduña-Rojas, J. \& Longoria-Espinoza, R.M. 2006. Metal content in Ulva lactuca (Linnaeus) from Navachiste Bay (southeast Gulf of California) Sinaloa, Mexico. Bulletin of Environmental Contamination and Toxicology, 77: 574-580.

Osuna-López, J.I., Frías-Espericueta, M.G., López-López, G., Zazueta-Padilla, H.M., Izaguirre-Fierro, G., PáezOsuna, F., et al. 2009. Cd, $\mathrm{Pb}$ and organochlorine pesticides of Mytella strigata (Pelecypoda: Mytilidae) of six coastal lagoons of NW Mexico. Boletín de Investigaciones Marinas y Costeras, 38(2): 233-239.

Osuna-Martínez, C.C., Páez-Osuna, F. \& AlonsoRodríguez, R. 2010. Mercury in cultured oysters (Crassostrea gigas Thunberg, 1793 and $C$. corteziensis Hertlein, 1951) from four coastal lagoons of the SE Gulf of California, Mexico. Bulletin of Environmental Contamination and Toxicology, 85: 339-343.

Osuna-Martínez, C.C., Páez-Osuna, F. \& AlonsoRodríguez, R. 2011. Cadmium, copper, lead and zinc in cultured oysters under two contrasting climatic conditions in coastal lagoons from SE Gulf of California, Mexico. Bulletin of Environmental Contamination and Toxicology, 87: 272-275.

Páez-Osuna, F. \& Osuna-Martínez, C. 2011. Biomonitors of coastal pollution with reference to the situation in the Mexican coasts: a review on the utilization of organisms. Hidrobiológica, 21(3): 229-238.

Páez-Osuna, F. \& Osuna-Martínez, C.C. 2015. Bioavailability of cadmium, copper, mercury, lead, and zinc in subtropical coastal lagoons from the Southeast Gulf of California using mangrove oysters (Crassostrea corteziensis and Crassostrea palmula). Archives of Environmental Contamination and Toxicology, 68: 305-316.

Páez-Osuna, F., Calderón-Campuzano, M.F., SotoJiménez, M.F. \& Ruelas-Inzunza, J. 2010a. Trace metals $(\mathrm{Cd}, \mathrm{Cu}, \mathrm{Ni}$, and $\mathrm{Zn})$ in blood and eggs of the sea turtle Lepidochelys olivacea from a nesting colony of Oaxaca, Mexico. Archives of Environmental Contamination and Toxicology, 59: 632-641.

Páez-Osuna, F., Calderón-Campuzano, M.F., SotoJiménez, M.F. \& Ruelas-Inzunza, J. 2010b. Lead in blood and eggs of the sea turtle, Lepidochelys olivacea, 
from the Eastern Pacific: concentration, isotopic composition and maternal transfer. Marine Pollution Bulletin, 60: 433-439.

Páez-Osuna, F., Calderón-Campuzano, M.F., SotoJiménez, M.F. \& Ruelas-Inzunza, J. 2011. Mercury in blood and eggs of the sea turtle Lepidochelys olivacea from a nesting colony in Oaxaca, Mexico. Marine Pollution Bulletin, 62: 1320-1323.

Páez-Osuna, F., Ruiz-Fernández, A.C., Botello, A.V., Ponce-Vélez, G., Osuna-López, J.I., FríasEspericueta, M.G., López-López, G. \& ZazuetaPadilla, H.M. 2002. Concentrations of selected trace metals $(\mathrm{Cu}, \mathrm{Pb}, \mathrm{Zn})$, organochlorines (PCBs, HCB) and total PAHs in mangrove oysters from the Pacific Coast of Mexico: an overview. Marine Pollution Bulletin, 44: 1296-1313.

Páez-Osuna, F., Álvarez-Borrego, S., Ruiz-Fernández, A.C., García-Hernández, J., Jara-Marini, M.E., Bergés-Tiznado, M.E., et al. 2017. Environmental status of the Gulf of California: a pollution review. Earth-Science Reviews, 166: 181-205.

Palomarez-García, J.M., Castañeda-Chávez, M.R., Lango-Reynoso, F. \& Landeros-Sánchez, C. 2009. Niveles de metales pesados en camarón café Farfantepenaeus aztecus de la laguna de Tamiahua, Veracruz, México. Revista de Investigaciones Marinas, 30(1): 63-69.

Pereira, M.E., Lillebø, A.I., Pato, P., Válega, M., Coelho, J.P., Lopes, C.B., et al. 2008. Mercury pollution in Ria de Aveiro (Portugal): a review of the system assessment. Environmental Monitoring and Assessment, 155: 39-49.

Pérez-Cruz, Y.G., Rangel-Ruiz, L.J. \& Gamboa-Aguilar, J. 2013. Metales en almejas y sedimentos en la Reserva de la Biósfera "Pantanos de Centla" Tabasco, México. Hidrobiológica, 23(1): 1-8.

Qiu, Y.W. \& Wang, W.X. 2016. Comparison of mercury bioaccumulation between wild and mariculture food chains from a subtropical bay of Southern China. Environmental Geochemistry and Health, 38(1): 3949.

Rabiul, G.M.I., Habib, M.R., Waid, J.L., Rahman, M.S., Kabir, J., Akter, S. \& Jolly, Y.N. 2017. Heavy metal contamination of freshwater prawn (Macrobrachium rosenbergii) and prawn feed in Bangladesh: a marketbased study to highlight probable health risks. Chemosphere, 170: 282-289.

RAMSAR. 2019. The list of wetlands of international importance. The Secretariat of the Convention on Wetlands (Ramsar, Iran, 1971), Gland.

Reyes, Y.C., Vergara, I., Torres, O.E., Díaz, M. \& González, E. 2016. Heavy metals contamination: implications for health and food safety. Revista Ingeniería, Investigación y Desarrollo, 16(2): 66-77.
Reyes-Montiel, N.J., Santamaría-Miranda, A., RodríguezMeza, G.D., Galindo-Reyez, J.G. \& GonzálezOcampo, H.A. 2013. Concentrations of organochlorine pesticides in fish (Mugil cephalus) from a coastal ecosystem in the southwestern Gulf of California. Journal of Environmental Biology, 113(3): $1-11$.

Riosmena-Rodríguez, R., Talavera-Sáenz, A., AcostaVargas, B. \& Gardner, S.C. 2010. Heavy metals dynamics in seaweeds and seagrasses in Bahía Magdalena, B.C.S., México. Journal of Applied Phycology, 22: 283-291.

Rivera-Rodríguez, L.B. \& Rodríguez-Estrella, R. 2011. Incidence of organochlorine pesticides and the health condition of nestling ospreys (Pandion haliaetus) at Laguna San Ignacio, a pristine area of Baja California Sur, Mexico. Ecotoxicology, 20: 29-38.

Robledo-Marenco, M.L. 2006. Presence of persistent organochlorine pesticides in estuaries of the subtropical Mexican Pacific. International Journal of Environment and Pollution, 26: 284-294.

Rodríguez, S.A., Ávila-Pérez, P. \& Velazquez-Olvera, I.A. 2001. Effects on the zooplankton community of pollutant disposal into the José Antonio Alzate dam, Mexico. International Journal of Environment and Pollution, 15(5): 497-504.

Rodríguez, A.R., Monks, S., Pulido, G., Gaytán, J.C., Romo, C. \& González, V. 2012. Metales pesados en el pez Dormitator Latifrons (Richardson, 1884) y agua de la Laguna de Tres Palos, Guerrero, México. Ra Ximhai, 8(2): 43-47.

Rodríguez-Amador, R., Monks, S., Pulido-Flores, G., Gaytán-Oyarzún, J.C. \& Romo-Gómez, C. 2014. Presence of lead and cadmium in Ariopsis guatemalensis (Günter, 1864), in the Tres Palos Lagoon, Guerrero, Mexico. Revista Científica Biológico Agropecuaria Tuxpan, 2(3): 551-555.

Ruelas-Inzunza, J. \& Páez-Osuna, F. 2004a. Distribution and concentration of trace metals in tissues of three penaeid shrimp species from Altata-Ensenada del Pabellón Lagoon (S.E. Gulf of California). Bulletin of Environmental Contamination and Toxicology, 72: 452-459.

Ruelas-Inzunza, J. \& Páez-Osuna, F. 2004b. Trace metals in tissues of resident and migratory birds from a lagoon associated with an agricultural drainage basin (SE Gulf of California). Archives of Environmental Contamination and Toxicology, 47: 117-125.

Ruelas-Inzunza, J. \& Páez-Osuna, F. 2005. Mercury in fish and shark tissues from two coastal lagoons in the Gulf of California, Mexico. Bulletin of Environmental Contamination and Toxicology, 74: 294-300. 
Ruelas-Inzunza, J. \& Páez-Osuna, F. 2006. Trace metal concentrations in different primary producers from Altata-Ensenada del Pabellón and Guaymas Bay (Gulf of California). Bulletin of Environmental Contamination and Toxicology, 76: 327-333.

Ruelas-Inzunza, J. \& Páez-Osuna, F. 2008. Trophic distribution of $\mathrm{Cd}, \mathrm{Pb}$, and $\mathrm{Zn}$ in a food web from Altata-Ensenada del Pabellón Subtropical Lagoon, SE Gulf of California. Archives of Environmental Contamination and Toxicology, 54: 584-596.

Ruelas-Inzunza, J., Escobar-Sánchez, O. \& Páez-Osuna, F. 2014. Mercury in fish, crustaceans and mollusks from estuarine areas in the Pacific Ocean and Gulf of Mexico under varying human. In: Amezcua, F. \& Bellgraph, B. (Eds.). Fisheries management of Mexican and Central American estuaries. Estuaries of the world impact. Springer, Berlin, pp. 39-49.

Ruelas-Inzunza, J., Gárate-Viera Y. \& Páez-Osuna, F. 2007. Lead in clams and fish of dietary importance from Coatzacoalcos Estuary (Gulf of Mexico), an industrialized tropical region. Bulletin of Environmental Contamination and Toxicology, 79: 508-513.

Ruelas-Inzunza, J., García-Rosales, S.B. \& Páez-Osuna, F. 2004. Distribution of mercury in adult penaeid shrimps from Altata-Ensenada del Pabellon Lagoon (SE Gulf of California). Chemosphere, 57: 1657-1661.

Ruelas-Inzunza, J., Hernández-Osuna, J. \& Páez-Osuna, F. 2009b. Organic and total mercury in muscle tissue of five aquatic birds with different feeding habits from the SE Gulf of California, Mexico. Chemosphere, 76: 415-418.

Ruelas-Inzunza, J., Hernández-Osuna, J. \& Páez-Osuna, F. 2011b. Total and organic mercury in ten fish species for human consumption from the Mexican Pacific. Bulletin of Environmental Contamination and Toxicology, 86: 679-683.

Ruelas-Inzunza, J., Meza-López, G. \& Páez-Osuna, F. 2008. Mercury in fish that are of dietary importance from the coasts of Sinaloa (SE Gulf of California). Journal of Food Composition and Analysis, 21: 211218.

Ruelas-Inzunza, J., Páez-Osuna, F. \& Soto, L.A. 2005. Bioaccumulation of $\mathrm{Cd}, \mathrm{Co}, \mathrm{Cr}, \mathrm{Cu}, \mathrm{Fe}, \mathrm{Hg}, \mathrm{Mn}, \mathrm{Ni}$, $\mathrm{Pb}$ and $\mathrm{Zn}$ in trophosome and vestimentum of the tube worm Riftia pachyptila from Guaymas basin, Gulf of California. Deep-Sea Research Part I, 52: 1319-1323.

Ruelas-Inzunza, J.R., Horvat, M., Pérez-Cortés, H. \& Páez-Osuna, F. 2003. Methylmercury and total mercury distribution in tissues of gray whales (Eschrichtius robustus) and spinner dolphins (Stenella longirostris) stranded along the lower Gulf of California, Mexico. Ciencias Marinas, 29(1): 1-8.

Ruelas-Inzunza, J., Páez-Osuna, F., Ruiz-Fernández, A.C. \& Zamora-Arellano, N. 2011a. Health risk associated to dietary intake of mercury in selected coastal areas of Mexico. Bulletin of Environmental Contamination and Toxicology, 86: 180-188.

Ruelas-Inzunza, J., Páez-Osuna, F., Zamora-Arellano, N., Amezcua-Martínez, F. \& Bojórquez-Leyva, H. 2009a. Mercury in biota and surficial sediments from Coatzacoalcos Estuary, Gulf of Mexico: distribution and seasonal variation. Water Air and Soil Pollution, 197: 165-174.

Ruelas-Inzunza, J., Sánchez-Osuna, K., AmezcuaMartínez, F., Spanopoulos-Zarco, P. \& ManzanoLuna, L. 2012. Mercury levels in selected bycatch fish species from industrial shrimp-trawl fishery in the SE Gulf of California. Marine Pollution Bulletin, 64(12): 2857-2859.

Ruelas-Inzunza, J., Delgado-Alvarez, C., FríasEspericueta, M. \& Páez-Osuna, F. 2013a. Mercury in the atmospheric and costal environments of México. In: Whitacre, D.M. (Ed.). Reviews of environmental contamination and toxicology, vol. 226. Springer, New York, pp. 65-100.

Ruelas-Inzunza, J., Escobar-Sánchez, O., Patrón-Gómez, J., Moreno-Sánchez, X.G., Murillo-Olmeda, A., Spanopoulos-Hernández, M. \& Corro-Espinosa, D. 2013b. Mercury in muscle and liver of ten ray species from Northwest Mexico. Marine Pollution Bulletin, 77: 434-436.

Saaristo, M., Brodin, T., Balshine, S., Bertram, M.G., Brooks, B.W., Ehlman, S.M., et al. 2018. Direct and indirect effects of chemical contaminants on the behaviour, ecology and evolution of wildlife. Proceedings of the Royal Society B, 285: 20181297.

Schneider, L., Maher, W.A., Potts, J., Taylor, A.M., Batley, G.E., Krikowa, F. \& Gruber, B. 2018. Trophic transfer of metals in a seagrass food web: bioaccumulation of essential and non-essential metals. Marine Pollution Bulletin, 131: 468-480.

Sedeño-Díaz, J.E. \& López-López, E. 2012. Freshwater fish as sentinel organisms: from the molecular to the population level, a review. In: Türker, H. (Ed.). New advances and contributions to fish biology. IntechOpen, London, pp. 151-173.

Segovia-Zavala, J.A., Delgadillo-Hinojosa, F., MuñozBarbosa, A., Gutiérrez-Galindo E.A. \& VidalTalamantes, R. 2004. Cadmium and silver in Mytilus californianus transplanted to an anthropogenic influenced and coastal upwelling areas in the Mexican Northeastern Pacific. Marine Pollution Bulletin, 48: 458-464.

Sobrino-Figueroa, F., Cáceres, M. \& Rosas, C. 2007. Evaluación del riesgo por consumir moluscos contaminados con cadmio, cromo y plomo. Hidrobiológica, 17(1): 49-58.

Soto-Jiménez, M., Páez-Osuna, F. \& Morales-Hernández, F. 2001. Selected trace metals in oyters (Crassostra 
iridescens) and sediments from the discharge zone of the submarine sewage outfall in Mazatlán Bay (southeast Gulf of California): chemical fractions and bioaccumulation factors. Environmental Pollution, 114: 357-370.

Soto-Jiménez, M.F., Páez-Osuna, F., Scelfo, G., Hibdon, S., Franks, R., Aggarawl, J. \& Russell, A. 2008. Lead pollution in subtropical ecosystems on the SE Gulf of California coast: a study of concentrations and isotopic composition. Marine Environmental Research, 66: 451-458.

Spanopoulos-Zarco, P., Ruelas-Inzunza, J., MezaMontenegro, M., Osuna-Sánchez, K. \& AmezcuaMartínez, F. 2014. Health risk assessment from mercury levels in bycatch fish species from the coasts of Guerrero, Mexico (Eastern Pacific). Bulletin of Environmental Contamination and Toxicology, 93: 334-338.

Stong, T., Alvarado, C., Shear, H., Anda, J., Ramírez, G. \& Díaz, J.J. 2013. Mercury concentrations in common carp (Cyprinus carpio) in Lake Chapala, Mexico: a lakewide survey. Journal of Environmental Science and Health, Part A, 48(14): 1835-1841.

Schwarzenbach, R.P., Escher, B.I., Fenner, K., Hofstetter, T.B., Johnson, C.A., Von Gunten, U. \& Wehrli, B. 2006. The challenge of micropollutants in aquatic systems. Science, 313(5790): 1072-1077.

Suárez-Serrano, A., Alcaraz, C., Ibáñez, C., Trobajo, R. \& Barata, C. 2010. Procambarus clarkii as a bioindicator of heavy metal pollution sources in the lower Ebro River and Delta. Ecotoxicology and Environmental Safety, 73: 280-286.

Talavera-Saenz, A., Gardner, S., Riosmena, R. \& Acosta, B. 2007. Metal profiles used as environmental markers of Green Turtle (Chelonia mydas) foraging resources. Science of the Total Environment, 373: 94-102.

Tejeda, S., Zarazúa, G., Ávila-Pérez, P., Carapia-Morales, L. \& Martínez, T. 2010. Total reflection X-ray fluorescence spectrometric determination of elements in water hyacinth from the Lerma River. Spectrochimica Acta Part B, 65: 483-488.

Terrazas-López, R., Arreola-Mendoza, L., GalvánMagaña, F., Anguiano-Zamora, M., Sujitha, S.B. \& Jonathan, M.P. 2016. Cadmium concentration in liver and muscle of silky shark (Carcharhinus falciformis) in the tip of Baja California south, México. Marine Pollution Bulletin, 107(1): 389-392.

Torres, Z., Mora, M.A., Taylor, R.J., Alvarez-Bernal, D., Buelna, H.R. \& Hyodo, A. 2014. Accumulation and hazard assessment of mercury to waterbirds at Lake Chapala, Mexico. Environmental Science \& Technology, 48: 6359-6365.

Trasande, L., Cortes, J.E., Landrigan, P.J., Abercrombie, M.I., Bopp, R.F. \& Cifuentes, E. 2010. Methylmercury exposure in a subsistence fishing community in Lake Chapala, Mexico: an ecological approach. Environmental Health, 9(1): 1-10.

Trillanes, C.E., Pérez-Jiménez, J.C., Rosíles-Martínez, R. \& González-Jáuregui, M. 2014. Metals in the caudal scutes of morelet's crocodile (Crocodylus moreletii) from the southern Gulf of Mexico. Bulletin of Environmental Contamination and Toxicology, 93: 423-428.

Uresti-Marín, R.M., Santiago-Adame, R., Díaz-Moroles, N.E., Gutiérrez-Lozano, J., Vázquez, M. \& Ramírez de León, J.A. 2008. Preliminary evaluation of the presence of organochlorine pesticides in fish of Vicente Guerrero dam (Tamaulipas, México). Ciencia y Tecnología Alimentaria, 6(1): 48-55.

Vaisman, A.G., Marins, R.V. \& Lacerda, L.D. 2005. Characterization of the mangrove oyster, Crassostrea rhizophorae, as a biomonitor for mercury in tropical estuarine systems, Northeast Brazil. Bulletin of Environmental Contamination and Toxicology, 74: 582-588.

Van der Oost, R., Beyer, J. \& Vermeulen, N.P.E. 2003. Fish bioaccumulation and biomarkers in environmental risk assessment: a review. Environmental Toxicology and Pharmacology, 13: 57-149.

Vargas-González, H.H., Méndez-Rodríguez, L.C., García-Hernández, J., Mendoza-Salgado, R.A., Zenteno-Savín, T. \& Arreola-Lizárraga, J.A. 2016. Persistent organic pollutants (POPs) in populations of the clam Chione californiensis in coastal lagoons of the Gulf of California. Journal of Environmental Science and Health Part B, 51(7): 1-11.

Vázquez, F.G., Sharma, V.K., Mendoza, Q.A. \& Hernández, R. 2001. Metals in fish and shrimp of the Campeche Sound, Gulf of Mexico. Bulletin of Environmental Contamination and Toxicology, 67: 756-762.

Vázquez-Boucard, C., Anguiano-Vega, G., Mercier, L. \& Rojas, E. 2014. Pesticide residues, heavy metals, and DNA damage in sentinel oysters Crassostrea gigas from Sinaloa and Sonora, Mexico. Journal of Toxicology and Environmental Health, Part A, 77(4): 169-176.

Vázquez-Sauceda, M.L., Aguirre-Guzmán, G., Pérez R., Rábago-Castro, J. \& Sanchez, G.J. 2005. Contenido de cadmio y plomo en agua, ostión y sedimento de la laguna de San Andrés, Aldama, Tamaulipas. Ciencia y Mar, 9(27): 3-9.

Vázquez-Sauceda, M.L., Aguirre-Guzmán, G., SánchezMartínez, J.G. \& Pérez-Castañeda, R. 2011. Cadmium, lead and zinc concentrations in water, sediment and oyster (Crassostrea virginica) of San Andres Lagoon, Mexico Bulletin of Environmental Contamination and Toxicology, 86: 410-414. 
Vélez-Alavez, M., Labrada-Martagón, V., MéndezRodríguez, L.C., Galván-Magaña, F. \& ZentenoSavín, T. 2013. Oxidative stress indicators and trace element concentrations in tissues of mako shark (Isurus oxyrinchus). Comparative Biochemistry and Physiology Part A: Molecular \& Integrative, 165: 508514.

Villanueva, F.S. \& Botello, A.V. 1992. Metales pesados en la zona costera del Golfo de México y Caribe Mexicano: una revisión. Revista Internacional de Contaminación Ambiental, 8(1): 47-61.

Waltham, N.J., Teasdale, P.R. \& Connolly, R.M. 2013. Use of flathead mullet (Mugil cephalus) in coastal biomonitor studies: review and recommendations for future studies. Marine Pollution Bulletin, 69: 195-205.

Wang, S., Xiang-Rong, X., Yu-Xin, S., Jin-Ling, L. \& Hua-Bin, L. 2013. Heavy metal pollution in coastal areas of South China: a review. Marine Pollution Bulletin, 76: 7-15.

Weng, N. \& Wang, W.X. 2017. Dynamics of maternally transferred trace elements in oyster larvae and latent growth effects. Scientific Reports, 7(3580): 1-11.

Wiener, J.G., Krabbenhoft, D.P., Heinz, G.H. \& Scheuhammer, A.M. 2003. Ecotoxicology of mercury. In: Hoffman, D.J., Rattner, B.A., Burton, G.A. \& Cairns, J. (Eds.). Handbook of ecotoxicology. Lewis Publishers, Boca Raton, pp. 409-462.

Zamora-Arellano, N.Y., Ruelas-Inzunza, J., GarcíaHernández, J., Ilizaliturri-Hernández, C.A. \& Betancourt-Lozano, M. 2017. Linking fish consumption patterns and health risk assessment of mercury exposure in a coastal community of NW Mexico. Human and Ecological Risk Assessment: An International Journal, 23(6): 1505-1521.

Received: 11 November 2019; Accepted: 21 July 2020
Zarazúa, G., Ávila-Pérez, P., Tejeda, S., ValdiviaBarrientos, M., Zepeda-Gómez, C. \& MacedoMiranda, G. 2013. Evaluación de los metales pesados $\mathrm{Cr}, \mathrm{Mn}, \mathrm{Fe}, \mathrm{Cu}, \mathrm{Zn}$ y $\mathrm{Pb}$ en sombrerillo de agua (Hydrocotyle ranunculoides) del curso alto del río Lerma, México. Revista Internacional de Contaminación Ambiental, 29(2): 17-24.

Zavala-Norzagaray, A.A., Ley-Quiñónez, C.P., EspinosaCarreón, T.L., Canizalez-Román, A., Hart, C.E. \& Aguirre, A.A. 2014. Trace elements in blood of sea turtles Lepidochelys olivacea in the Gulf of California, Mexico. Bulletin of Environmental Contamination and Toxicology, 93: 536-541.

Zhang, R., Wu, F., Li, H., Guo, G., Feng, C., Giesy, J.P. \& Chang, H. 2013. Toxicity reference values and tissue residue criteria for protecting avian wildlife exposed to methylmercury in China. Reviews of Environmental Contamination and Toxicology, 223: 53-80.

Zorita, A.I, Ortiz-Zarragoitia, M., Orbea, A., Cancio, I., Soto, M., Marigómez, I. \& Cajaraville, M. 2007. Assessment of biological effects of environmental pollution along the NW Mediterranean Sea using mussels as sentinel organisms. Environmental Pollution, 148: 236-250. 